\title{
A Formative Study of the Implementation of the Inquiry Team Process in New York City Public Schools: 2007-08 Findings
}

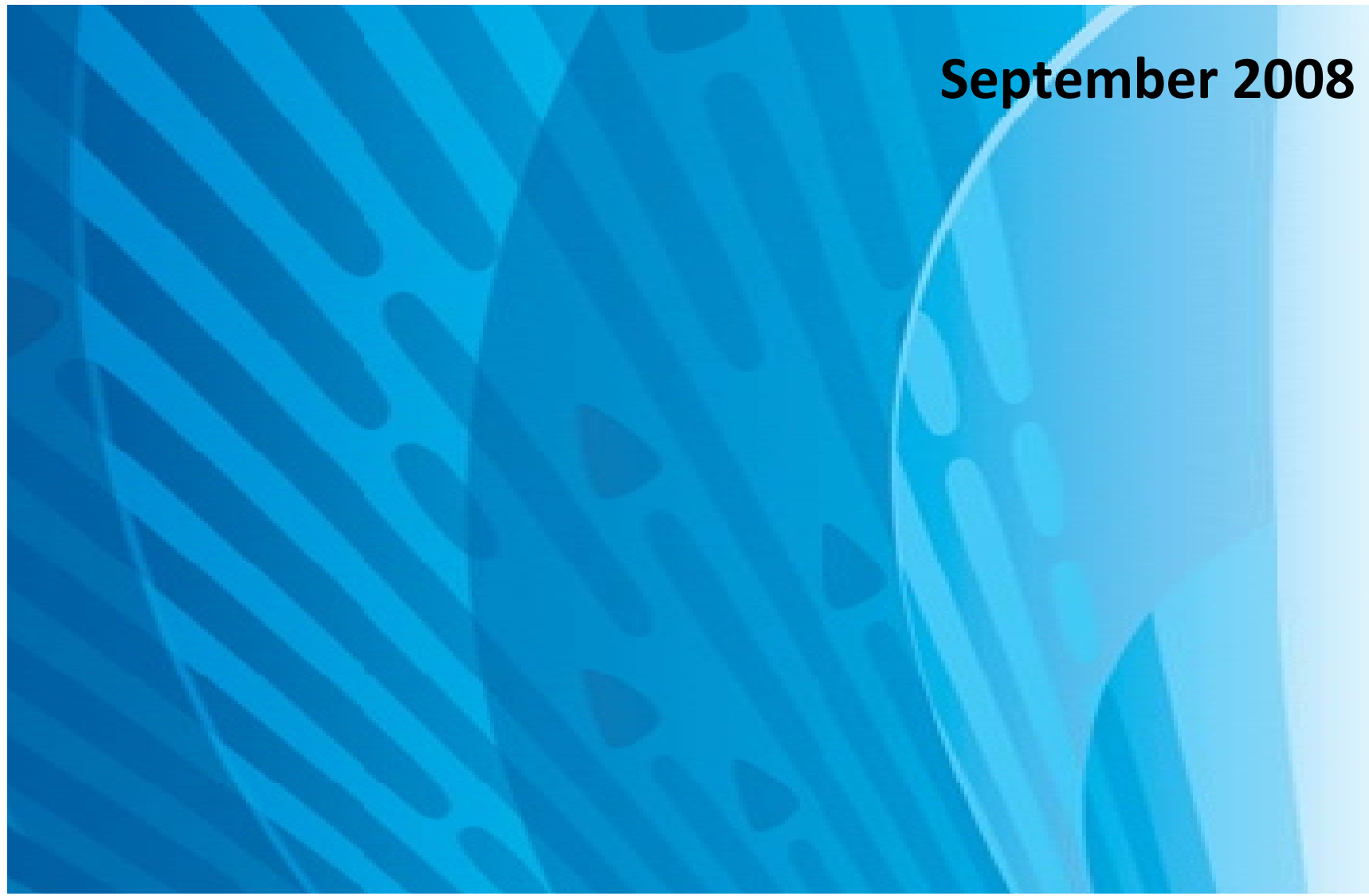

Prepared by:

Marian A. Robinson

Patricia Kannapel Joan Gujarati Hakim Williams Andrea Oettinger

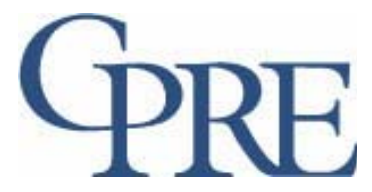




\section{About the Consortium for Policy Research in Education (CPRE)}

Established in 1985, CPRE unites researchers from seven of the nation's leading research institutions in efforts to improve elementary and secondary education through practical research on policy, finance, school reform, and school governance. CPRE studies alternative approaches to education reform to determine how state and local policies can promote student learning. The Consortium's member institutions are the University of Pennsylvania, Teachers College-Columbia University, Harvard University, Stanford University, the University of Michigan, University of Wisconsin-Madison, and Northwestern University.

In March 2006, CPRE launched the Center on Continuous Instructional Improvement (CCII), a center engaged in research and development on tools, processes, and policies intended to promote the continuous improvement of instructional practice. CCII also aspires to be a forum for sharing, discussing, and strengthening the work of leading researchers, developers and practitioners, both in the United States and across the globe.

To learn more about CPRE and CCII, visit our web sites at www.cpre.org and www.ccii-cpre.org. 


\title{
A Formative Study of the Implementation of the Inquiry Team Process in New York City Public Schools: 2007-08 Findings
}

September, 2008

Prepared by

Marian A. Robinson

Patricia Kannapel

Joan Gujarati

Hakim Williams

Andrea Oettinger

\author{
Consortium for Policy Research in Education \\ Teachers College, Columbia University
}

All data presented, statements made, and views expressed in this report are the responsibility of the authors and do not necessarily reflect the views of the Consortium for Policy Research in Education, its institutional partners, or the funders of this study-The Eli and Edythe Broad Foundation and the Robertson Foundation. This report has been internally reviewed by members of CPRE research staff; however, it has not been externally reviewed. 



\section{Table of Contents}

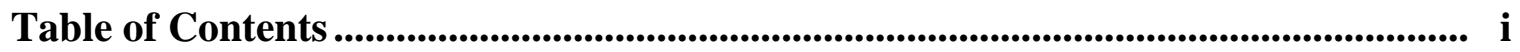

Acknowledgments ............................................................................................................................... iii

Executive Summary ................................................................................................................... V

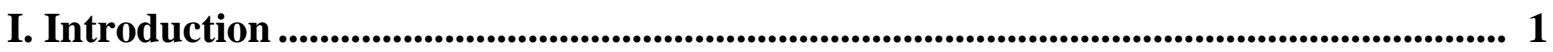

The Inquiry Team Process

Sources of Support for Inquiry Teams

II. Research Design and Methods................................................................................................. 9

Study Sample

Data Collection

Data Analysis

Report Overview

III. Key Features of School-Based Inquiry Teams ...................................................................15

Team Size, Composition, and Stability

The Role of the Principal

The Role of the Data Specialist

Team Dynamics

IV. How Teams Approached the Inquiry Process

The Pace and Completion of the Inquiry Team Work

Identifying a Schoolwide Focus

Identifying a Target Population

Developing and Implementing Change Strategies

Evaluating the Effectiveness of Change Strategies

Sharing the Work of the Inquiry Team

Stimulating Larger Improvements in the School

Sustaining and Deepening the Work Within the School

Summary

V. External Support for the Inquiry Team Process

The Contributions of Senior Achievement Facilitators

The Contributions of SSO Networks to the Inquiry Team Process

The Network Contexts for Supporting Inquiry Teams 
VI. Accomplishments of the Inquiry Team Process in 2007-08...................................73

Completing a Full Cycle of the Inquiry Team Process

Developing Team Capacity to Implement the Inquiry Team Process

Increasing Commitment to Data-Informed Inquiry

Influencing Teacher Practice

Stimulating School Improvements

Summary

VII. Areas of Strength and Need in 2007-08..................................................................91

Areas of Strength

Areas of Need

VIII. Recommendations

\begin{abstract}
Appendices
A. Data Collection Protocols: School Level

B. Data Collection Protocols: External Support Structure

C. School Profile Data Summary Template

D. Inquiry Team Composition

E. Team Progress Towards Their June 2008 Learning Goals

F. Department Plans to Strengthen the Inquiry Team Process for 2008-09
\end{abstract}

\title{
Figures and Tables
}

Figure 1. The Inquiry Team Process ..................................................................... 5

Figure 2. Organizational Structure Supporting School-Based Inquiry Teams ................... 6

Figure 3. Team Member Descriptions of Different Levels of SAF Support ........................64

Figure 4. Full Implementation of the Inquiry Team Process by a Year One School.............77

Table 1. Characteristics of the Study's General Education School Sample Compared With the New York City School System................................... 11

Table 2. Qualitative Data Supporting the Study by Level ............................................ 12

Table 3. Variation in Team Composition by Member Role in the School ..........................19

Table 4. Variation in the Role of the Principal in the School Inquiry Team .......................21

Table 5. Overlap Between the Data Specialist Position and Other School Roles ................25

Table 6. Team Meeting Observation Ratings by Principal and Data Specialist Roles..........30

Table 7. Variation in the Type and Intensity of SAF Support .........................................63

Table 8. Variation in Interactions Between Inquiry Teams and Network Staff .................66

Table 9. Variation in Implementation Status Across Study Schools During the Final 


\section{Acknowledgments}

A study of this scale and conducted in such a condensed time frame is only made possible through the effort and commitment of many individuals. We are grateful to the hundreds of educators and administrators who took time from their busy schedules to share their views and experiences implementing the Inquiry Team process. Scores of Senior Achievement Facilitators and SSO Network Leaders - all managing demanding work schedules and extra end-of-year responsibilities - also made time to share their experiences supporting this new initiative. Special thanks are extended to CFI leadership team members at the Department who were always available with sensible guidance, incisive questions, and a wealth of information that helped jump-start the study and keep it on track through its intensive four months. On the research side, Henry May designed and implemented the methodology used to draw the sample of schools for the study. We want to acknowledge the special contributions of researchers Claire Passantino and Keisha McIntosh, whose fieldwork and preliminary data analysis greatly informed the preparation of this report. The study benefited from the advice (and wisdom) of Tom Corcoran and Leslie Santee Siskin, whose feedback informed the study's framework and data collection instruments. Finally, Connie Langland edited the report and we are grateful for her flexibility and responsiveness during that process.

This study was made possible in part with support from The Eli and Edythe Broad Foundation and the Robertson Foundation.

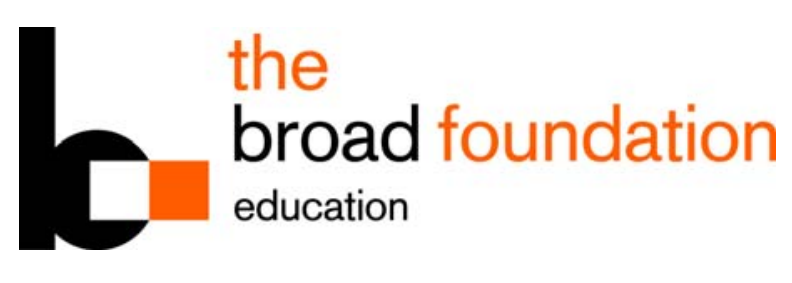

\section{ROBERTSON}


Formative Study of the NYC Inquiry Team Process, 2007-08 


\section{First-Year Citywide Implementation of the Inquiry Team process in NYC Schools: Executive Summary of the CPRE Study}

During the 2007-08 school year, the New York City Department of Education launched the second phase of its ambitious and innovative initiative known as Children First. The reform is guided by three principles: leadership, empowerment, and accountability. Key components include restructuring central-office services, increasing principal authority, providing schools access to new forms and sources of support through affiliate school support organizations (SSOs), and developing new data-driven accountability tools that hold schools responsible for student performance while providing rich diagnostic information to support organizational and instructional improvement. Ultimately, this initiative aims to intensify the focus by all educators on student performance and in doing so to stimulate the development of evidence-based cultures at all levels of the system.

This report provides formative feedback on the implementation of an important new component of Children First called the Inquiry Team process. The Inquiry Team initiative was rolled out in all 1,450 city schools in the summer of 2007 after being piloted in Empowerment Schools in 2006-07. Under this initiative, schools are expected to establish small "Inquiry Teams" of teachers and administrators charged with examining the performance problems of small, select target populations of students with the goal of identifying instructional "change strategies" that will improve their performance. Through this process, these small teams are expected to make use of performance data and other information to diagnose and monitor student learning and to make recommendations for changes in any aspect of their school to help close the achievement gap for these target populations of low-performing students. Teams are expected to become experts in the diagnostic use of the accountability tools and other data and the Inquiry Team process itself. The goal is for teams to share their new knowledge with the broader school community throughout the year to simulate instructional changes and improvements in the conditions of teaching and learning.

\section{Focus of the Study: Assessing Implementation and Support}

Commissioned in April, 2008, the goals of this qualitative study were to provide quick formative feedback to the Department to help strengthen the Inquiry Team process and the quality of supports available to schools. The study focused on two key aspects of the process: (1) how schools, specifically team members and principals, have implemented the Inquiry Team process and (2) how the new external support organizations and personnel have approached supporting school-based teams. Data supporting this report come from interviews with principals and Inquiry Team members and team observations in 41 schools as well as interviews with affiliated SSO Network Leadership and Department Senior Achievement Facilitators (SAFs) charged with providing support to the Inquiry Teams. 
Information from interviews and observations reveals that the vast majority of schools in the CPRE study made substantial progress implementing the Inquiry Team process in 2007-08. By the time of the study, late in the school year, the great majority of team members understood the purpose and steps of the Inquiry Team process in ways that closely matched the Department's vision. The majority of schools had worked through the steps of the process with fidelity, and had seen the positive results of their work. A large majority of team members found the process to be of value, and had begun making plans for continuing or expanding the work in 2008-09.

There is room for improvement: Some teams felt frustrated and overwhelmed as a result of the workload, and some were adamant that for the process to succeed the Department would need to continue funding the work and provide even more support for implementation. In 2008-09, the Department may need to give particular attention to helping schools develop change strategies, and assisting them in using what they learned to effect schoolwide changes - two underdeveloped steps of the process in 2007-08.

There were some promising accomplishments during the first year of citywide implementation of the Inquiry Team process. A brief summary follows:

- By the time of CPRE site visits in late spring, 54\% of schools had implemented all major stages of the Inquiry Team process. This percent rises to $75 \%$ if we extend the implementation cycle into the early part of the 2008-09 school year.

- Members generally felt the Inquiry Team process was doable given time and support. Many teams were eager to improve and expand their work in 2008-09.

- The study identified a set of capacities that teams identified as important, including attitudes and beliefs about the inquiry process, and skills and knowledge associated with teamwork and data analysis. Evidence reflected that these capacities were present in various levels in three-quarters of the teams.

- The study found evidence of strong commitment to data use, in general, and the Inquiry Team process, in particular, related to the opportunity to identify effective change strategies and to share "what works" with teachers. A third of teams in this study promoted the value of data analysis in their schools and modeled, as a team, how data analysis can inform instruction and other improvements.

- A large percent of teachers, about $75 \%$, felt participation on the Inquiry Team had influenced their practice, and most were willing to continue on the team and advocate for more teacher participation.

- Teams reported mixed attention to taking a role in stimulating school improvements. Only about half of teams attempted to influence teacher practice or the wider systems that compose the school. This stage of the Inquiry Team process was the most difficult to understand and engage by teams in 2007-08. 


\section{Findings: Areas of Strength and Need}

The primary strengths observed by the CPRE research team include

Strong momentum going into 2008-09. Almost all of the teams conveyed a strong belief that they could do an even better job in the second year and were expecting to "hit the ground running" in September, by offering professional development to faculty.

Establishment of the Senior Achievement Facilitator role. A majority of Inquiry Teams credited much of their professional growth, progress, and accomplishments to the support they received from their SAFs. Issues included large SAF workloads and the tendency of some principals to test their new "empowerment" by declining support.

Establishment of the Data Specialist role. It is highly unlikely that so many teams could have completed the implementation cycle without the continuous school-based support provided by the Data Specialists. Still, almost all of the Data Specialists required training in Department systems, and some needed help creating data displays to facilitate team analysis.

Small-scale elements of the Inquiry Team process. Focusing on a single problem, getting to know a small group of students, working with a small group of committed, knowledgeable educators and administrators - these were powerful design elements of the Inquiry Team process. The small elements of the design helped members stay focused and see the inquiry process as doable, and created personal connections at both the topic and student levels that motivated teachers.

Positive, collaborative team dynamics. A large percentage of teams reported developing strong working relationships that were collaborative and highly grounded in the elements of the Inquiry Team process. Diverse, cross-role teams that were balanced in their composition tended to have the expertise, authority, and leadership needed to implement the Inquiry Team process effectively.

Contributions of Principals. Many Inquiry Teams were high functioning, due in part to principals who took a facilitative role in their work.

Clear alignment with other accountability tools. Team members saw a close alignment between their work and the standards against which schools were being measured on the Quality Review and Progress Report. The integration of these multiple tools was a strong factor in convincing educators of the value and seriousness of the Inquiry Team process.

The CPRE research team also noted areas of the process that need attention, including

Linking SSO resources to the Inquiry Team process. School support organizations have a wealth of instructional expertise and offer professional-development opportunities in mathematics, English, and programs for special student populations. Yet the SSOs have not systematically connected relevant resources to the school-level Inquiry Teams.

Executive Summary 
Ensuring teacher participation in the process. The value of having classroom teachers on the Inquiry Teams is widely acknowledged by both the Department and many team members, yet only $25 \%$ of team members were classroom teachers in the study's school sample in 2007-08. Also, the ambiguity of the role of target-population teachers - as targets or as formal team members_-posed both technical and political challenges to the process. Expanding teacher participation likely will require creative scheduling and shifts in resources at the school level.

Developing and implementing change strategies. Teams were most data-informed in selecting and diagnosing student needs in their school. They were less empirically oriented when selecting or developing strategies to address those learning needs. SAFs and SSO Network staffs could help teams search for and implement change strategies.

Material guidance on the tasks and pace of the Inquiry Team process. Information about the process was rolled out across the year, and supporting materials often were developed just in time as teams reached a given stage. Teams wanted a timeline to help them pace their work, and they wanted to know what other schools were doing. The availability of such guidance would minimize uncertainty and frustration and help teams focus.

Support for the use of low-inference transcripts. Team members, teachers in particular, stated that the opportunity to observe other teachers helped them reflect on their own instruction. The Inquiry Teams could be a vehicle for widespread use of low-inference transcripts if they gain access to better tools and guidance in their use.

Use of ARIS as a major resource. Interviews revealed that team members and Data Specialists hold high hopes for ARIS despite complaints about accessibility, use of passwords, the timing and utility of training, and website reliability and capacity.

Team focus on stimulating systemic change. Almost all teams had members who believed the ultimate goal of the Inquiry Team process was to bring about systemic changes in their school, yet only half of the teams in this study were able to promote recommendations for improving instruction or policies to others in the school during the 2007-08 school year. Among team members, there was awareness this was an undeveloped aspect of their work.

\section{Eight Recommendations}

1. Continue funding school-based Data Specialists and SAFs, who will bring experience and know-how to the process as Inquiry Teams expand. The SAF and Data Specialist positions were critical to the progress of the Inquiry Teams in this study. Data Specialists were important links by which Network Leaders and/or SAFs could monitor school needs and other developments affecting the work of the teams. The SAF position provided critical guidance about the process itself and other supports.

\section{Request that SSOs align their support and professional development to better support} the work of the Inquiry Teams. Most SSOs offer services or have capacity that can speak directly to Inquiry Team needs, specifically identifying change strategies. In some cases, it is a question of packaging and advertisement beyond principals to reach the teams directly. In other 
cases, SSOs will need to adapt or expand their in-house expertise to provide more support.

3. Develop a broad system of resources to support Inquiry Teams. The elements for building a coherent system of supports are available through ARIS, many SSO services, and some Webbased research databases. These need to be aligned with the focus and timing of the Inquiry Team process. Data Specialists and SAFs can help schools make use of these services and can populate databases with recent Inquiry Team experiences.

4. Reinforce the value of diverse teams that draw from multiple role groups in the school, specifically classroom teachers, nonclassroom-based instructional staff, and administrators. Many teams reflected a diverse composition, which resulted in a strong group dynamic when coupled with a chair who focused on facilitating, not directing, the team's work.

5. Encourage Inquiry Team chairs, Data Specialists, and external support staff to help teams anticipate problem areas at different points in the process. The CPRE study uncovered numerous issues that teams likely may address, including bolstering team expertise, designing and implementing the change strategy, monitoring change-strategy implementation, and informing faculty about the team's work.

6. Give Inquiry Teams opportunities to learn from other teams. The public sharing of work brings a level of face-to-face accountability for teams that can raise the level of seriousness about the work, and help them see new possibilities. Team members should have opportunities to participate in networking meetings, which should reinforce messages about good practice and data-oriented decision-making.

7. Provide guidance about good practice related to the Inquiry Team process and advice about pacing to enable completion of a full cycle in a single school year. There is strong demand for more guidance about the options, tradeoffs, and effective practices associated with different decision points in the process, and for guidance about pacing. Teams may benefit from a narrative overview of the Inquiry Team process, pacing guidance, descriptive case studies, and informational videos or professional development sessions.

8. Focus on strengthening the professional cultures of schools with little history of collaborative exchanges among teachers or between administration and faculty.

The likelihood of success using the Inquiry Team process is highly dependent on the quality of adult relationships in the school. Schools with fractious faculty relationships or that were just beginning to build trust around instructional conversations struggled to adopt the Inquiry Team process. Schools with such issues should be identified and quickly supported, since the process itself can illustrate the power of collaborative work relationships focused on student learning. 
Formative Study of the NYC Inquiry Team Process, 2007-08 


\section{A Formative Study of the Implementation of the Inquiry Team Process in New York City Public Schools: 2007-08 Findings}

\section{Introduction}

During the 2007-08 school year, the New York City Department of Education launched the second phase of its ambitious and innovative restructuring initiative known as Children First. This initiative aims to transform the culture and operation of both the city's schools and the administrative structures of the Department. The reform is guided by three principles: leadership, empowerment, and accountability. Key components include restructuring central-office services; increasing principal authority over staffing, resources, and educational decisions; providing schools access to new forms and sources of support through affiliate school support organizations (SSOs); and developing new data-driven accountability tools that simultaneously hold schools responsible for how effectively they improve each child's learning outcomes and provide rich diagnostic information to support organizational and instructional improvement. Under this initiative, schools will have greater freedom to make decisions as well as encounter fewer bureaucratic hurdles as they seek to improve their performance. In turn, principals will be held more accountable for their schools' performance and provided with the data and other tools necessary to use knowledge and evidence to drive improvement in school structures and outcomes. Ultimately, this restructuring initiative aims to shift the focus to student performance and in doing so to stimulate the development of evidence-based cultures at all levels of the system.

This report provides formative feedback on the implementation of an important new component of the Department's restructuring initiative called the Inquiry Team process, which was rolled out in all 1,450 city schools in the summer of 2007 after being piloted in the 300 Empowerment Schools in 2006-07. Under this initiative, schools are expected to establish small "Inquiry Teams" of teachers and administrators who are charged with examining the performance problems of small, select populations of students in their schools with the goal of identifying instructional strategies that will improve their performance and can subsequently be shared with the broader school community. Through this process, these small teams are expected to make use of performance data and other information to diagnose and monitor student learning and to make recommendations for changes in any and all aspects of their school to help close the achievement gap for these target populations of low-performing students. Additionally, the teams are expected to become experts in the diagnostic use of the accountability tools and other data and the Inquiry Team process itself. It is hoped that inquiry team members will become advocates and supports for the extension of the empowerment and accountability reforms and associated culture change to all professionals in their school buildings. 
In April, 2008, the Consortium for Policy Research in Education (CPRE) was invited by the New York City Department of Education to document the implementation of this promising initiative and to provide quick formative feedback to the Department to help strengthen the Inquiry Team process and the quality of supports available to schools. The study has focused on two key aspects of the Inquiry Team process: (1) how schools, specifically team members and principals, have implemented the Inquiry Team process and (2) how the new external support organizations and personnel have approached supporting school-based Inquiry Teams. Data supporting this report come from interviews with principals and Inquiry Team members and team observations in 41 schools as well as interviews with SSO Network Leadership and Senior Achievement Facilitators (SAFs) providing support to Inquiry Teams. This report follows a preliminary analysis provided to the Children First Initiative (CFI) leadership team in June.

\section{The Inquiry Team Process}

The Inquiry Team process offers schools an improvement strategy that is designed to generate new knowledge within each school and encourage school-based educators to take action based on that new knowledge to address barriers to student learning. Each school's Inquiry Team process begins with intensive study of the learning progress of a small group of students in their building. Central to each team's deep self-study of the quality of teaching, learning, and the environment of their school is an ongoing analysis of quantitative and qualitative data and each team member's own knowledge of the school and its students. Over time, it is expected that the increase in Inquiry Team members' capacity to use self-study to revise instructional practices not working for particular children, and the expansion in the number of Inquiry Teams in each school, will create a culture of responsibility for student outcomes and confidence among professionals to diagnose and correct instructional failings. The ultimate goal of the Inquiry Team process is to improve outcomes for all students.

Key Stages and Tasks of the Inquiry Team Process. The inquiry process is guided by a series of specific tasks and decision points that teams must complete. These are based on the team's own investigation, hypothesis testing, and reflection on the learning conditions and progress of a particular set of students at the school. A graphic of the Inquiry Team process is provided in Figure 1. Within the broad parameters of this process, teams have wide discretion to shape the focus of their work and to determine the data most relevant to their investigation of any problem of student learning at their school. Teams work through the following tasks ${ }^{1}$ :

- Defining a Schoolwide Focus for the Work of the Inquiry Team. To focus its work, each team first identifies a content area and a subpopulation of students who are struggling within that area. Teams are encouraged to focus on the lowest-performing third of students in their school. The selected subpopulation may be identified by grade level or special student population, or both. It should be a specific group of students for whom the school will be committed to accelerating learning. A team

\footnotetext{
${ }^{1}$ This description draws upon Department graphical displays and explanations of the Inquiry Team process.
} 
might decide, for example, to focus on $9^{\text {th }}$ grade students who had scored from 1 to 2.5 on the New York State English/Language Arts (ELA) tests for the last three years_-possibly a group of about 100 students.

- Selecting a Target Population of Students. To focus its work further, the team next examines this subpopulation of students more closely to identify a smaller, manageable group of students who are experiencing the types of learning difficulties faced by many students in the schoolwide focus area. This target population of students would typically be experiencing a common set of barriers to learning that the team identifies as it conducts a deeper analysis of the group's performance using standardized assessments as well as students' class work. Through this analysis, the team would search for patterns of more specific instructional challenges or weaknesses associated with a particular skill (e.g., teaching reading) and subskill (e.g., reading comprehension) in need of strengthening for the targeted group of students. The team then reviews this final group to identify a clear target population of 15-30 students who are judged likely to be responsive to the school's efforts to reach them - namely those students with regular attendance and few behavior issues.

- $\quad$ Setting Specific Learning Goals for the Target Population. Next, each team sets learning goals that the target population will reach by the end of the school year and identifies appropriate assessments to measure growth in the group's performance.

- Analyzing the Target Population's Conditions of Learning. The team next begins a systematic study of the target population by analyzing the current learning conditions of these students. This can include a review of curriculum, classroom interactions and environments, student scheduling policies, teacher assignment, the pedagogical methods used to teach the skill or subskill, and the quality of instructional delivery. From this investigation, the team identifies the instructional, material, organizational, and policy areas that need attention in order to strengthen the specific skill deficits being addressed for the target population students.

- Defining and Implementing an Instructional Change Strategy to Address Student Learning Needs. The team then takes action on this new knowledge to address one or more of the identified barriers to student learning. Team action takes the form of an instructional "change strategy" that aims to address one or more of the learning conditions identified in the team's analysis. It should be a change strategy that, while small, could make a big difference in terms of student performance. It is expected that the change strategies will differentiate instructional practice based on individual learning needs within the target population.

- Analyzing the Decision-Making Systems That Shape the Conditions of Learning. The team also takes time to step back to consider the school's policies and decisionmaking systems that shape the learning conditions for the target population. These policies and systems may include the curriculum, teacher and student scheduling, pedagogy, time allocations, and norms or standards of practice. Teams may determine that a larger problem needs to be addressed to improve the conditions of learning. 
- Defining and Implementing a System-Level Change Strategy to Address the Conditions of Learning. The team then takes action by identifying a system-level "change strategy" that aims to implement a small change in the decision-making system that can make a big difference in student learning across the school.

- Evaluating the Effectiveness of the Change Strategy. As the team implements the change strategies, members begin reflecting upon and revising the strategies as needed. To inform this reflection, the team gathers additional data to measure the effectiveness of the change strategy on the target population students' learning. This includes using interim assessments or other relevant data to monitor changes in student learning at specific intervals. It also includes considering potential explanations for strong or weak student progress by assessing how well the change strategies are being implemented, the strength of the change strategies themselves, or the appropriate level of student engagement. Teams take action to adjust their change strategies based on feedback from student assessments or other sources.

- Sharing the Work of the Inquiry Team. Teams are expected to share their work with other teachers in their schools throughout the school year. This includes introducing the purpose of the team to the wider population of educators and administrators in the school, and sharing ongoing research, analysis, and suggestions for improvements. By regularly sharing their work, it is hoped the teams will foster a culture of evidencebased decision-making in their schools, support extension of the inquiry model to other professionals in subsequent years, and promote a reflective approach to school improvement that is grounded in the unique context of each team's school. Conversations with the larger community about the specific change strategies that teams are pursuing have the potential to stimulate systemic school change and improve student learning. The Department has been taking steps to facilitate the sharing of problems and solutions among schools across the City through the implementation of a major content management and collaboration platform.

As Figure 1 suggests, the Inquiry Team process emphasizes data-informed decisions, offers team great discretion in focusing their work, is highly contextualized to the needs of students currently in a given school, and provides opportunities for considering connections between the learning conditions of specific students and the larger decisionmaking systems that frame the school's learning environment.

The first task is for principals to identify a small group of educators to lead this work in their schools. The Department has recommended teams be composed of 3-6 members, depending on the size of the school. Members should include the principal, classroom teachers (who should comprise a majority of the team), and other members possibly including assistant principals, coaches, and other "strong staff." Principals are encouraged to select individuals with "strong leadership capacity." One team member also serves as the Data Specialist and is expected to become familiar with and promote the use of the Department's data-reporting and knowledge-management system, ARIS (discussed later in this section), both within the team and more broadly within the school. 
Schools received a job description to facilitate the application process and additional funding to compensate Inquiry Team members for their time. Teams are expected to establish a common meeting time.

Figure 1. The Inquiry Team Process.

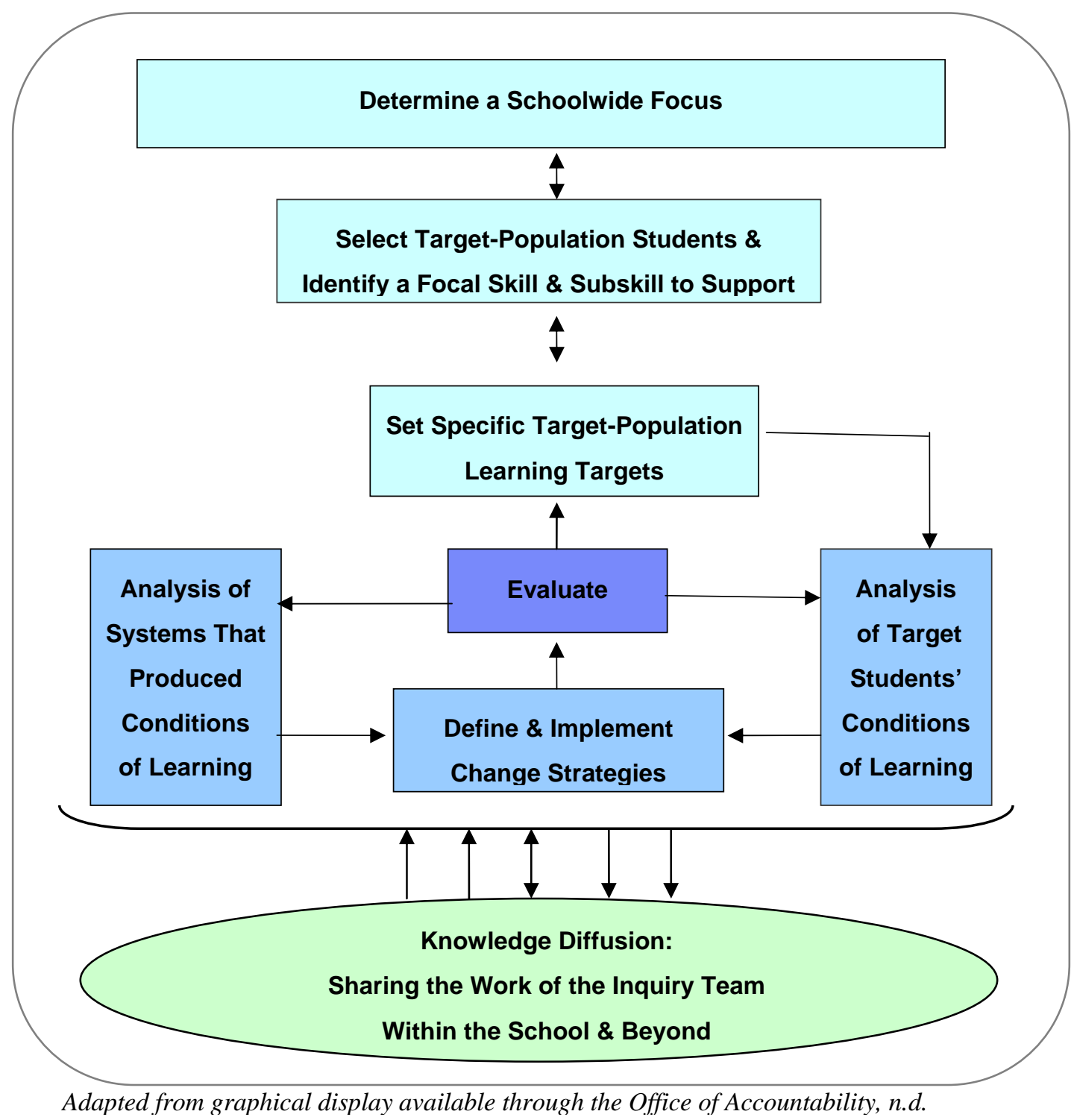

The Department also provided a timeline for schools to establish their Inquiry Teams. It was suggested that teams hold their first meeting by September 28, 2007, attend Inquiry Team training starting October 4, 2007, and select their target population of 15-30 students by November 15, 2007. 


\section{Sources of Support for Inquiry Teams}

The Department has identified a variety of material and human supports for the schoolbased Inquiry Teams. These supports are provided directly by the Department and through the new school support organization structure established in the summer of 2007.

Perhaps the most important resources for teams are the staffs situated within the new external support structure. Each school chose to affiliate with one of 11 new SSOs serving the city. There are three types of SSOs: the Empowerment Support Organization (ESO), the Partnership Support Organization (PSO), and the Learning Support Organization (LSO). Each SSO has a unique mission and provides a portfolio of instructional, business, legal, and consultative services and products to member schools, often including opportunities to network with other schools. Within each SSO, member schools are organized into smaller networks of schools. Typically, principals choose and/or approve their Network Leader who is charged with providing network schools technical assistance, networking opportunities, and other SSO services.

Each school also is assigned one of 64 Senior Achievement Facilitators, a new position established by the Office of Accountability. The SAFs are paired with SSOs and their school networks to work intensively with groups of 20-25 schools to support and facilitate the work of the local Inquiry Team, among other duties. As a representative of the Office of Accountability, SAFs are responsible for helping schools access, understand, and use all of the new accountability tools - including Quality Reviews, Progress Reports, and ARIS - to identify effective practices. The organizational relationships between the support structure and the school-based Inquiry Teams is provided in Figure 2.

Figure 2. Organizational Structure Supporting School-Based Inquiry Teams.

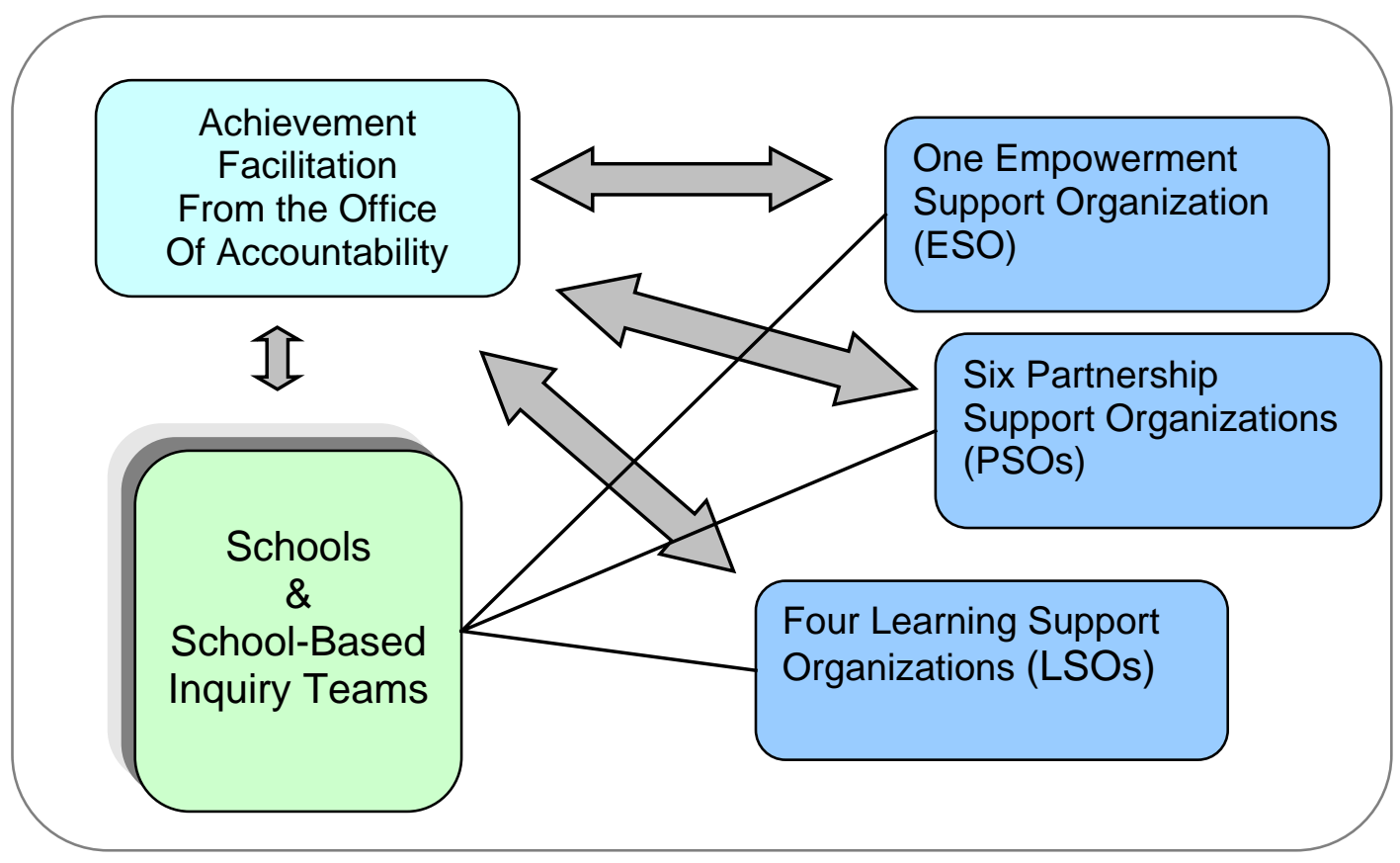

Source: Children First Intensive Summer Planning, Taking the Accountability Initiative Citywide, PowerPoint, Summer, 2007. 
Coordination between SSO Network Leaders and SAFs is expected to take place at a number of levels. Within each SSO, a planning group has been formed to bring together Lead SAFs and SSO instructional leadership for dialogue around the school Inquiry Teams and the accountability tools. For each school network, meetings of Collaborative Achievement Teams (CATs) bring together the Network Leader, SSO instructional staff, and the assigned SAF(s) to coordinate school support. Across the CPRE sample, SSO networks varied in size from 16 to 26 schools.

To support their work, school-based Inquiry Teams also have access to a host of new data, assessment, and collaboration tools that have been rolled out in the last two years. These tools include

- Progress Reports: This report provides each school with an analysis of the school's contribution to student academic performance, with attention to the progress of lowperforming students. A school's Progress Report grade is based on the following factors: school environment (15\%), student performance (25\%), student progress $(60 \%)$, and closing the achievement gap (additional credit). Progress reports help the Inquiry Team identify an area of need for its schoolwide focus.

- Quality Reviews: These qualitative assessments are in-depth profiles of each school based on a 2- to 3-day visit by experienced educators. During these visits, the reviewer talks to parents, students, and staff; observes classrooms; and reviews how the school uses information and sets goals to improve learning for all students. The work of the Inquiry Team feeds into a school's preparation for the Quality Review and is aligned with the review's emphasis on data-informed cultures of inquiry.

- Achievement Reporting and Innovation System (ARIS): This data-reporting and knowledge-management system is being established to enable parents and educators to see and analyze a broad range of data on student learning. ARIS promises to streamline user access to information currently stored in different data bases. ARIS also offers a collaboration tool with a resource library, blogs, discussion threads, Wikis, and calendars to facilitate information-sharing within and across schools, and potentially across Inquiry Teams. ARIS also provides links to Department assessment vendors. For Inquiry Teams, ARIS may provide a central resource for all aspects of their work.

- Inquiry Target Tool (ITT): The tool combines the student-level data in the Progress Report and data on current students in the Department's Automated Tracking System (ATS). It provides summary data on Progress Report metrics for different subpopulations within a given school. The ITT can help teams identify their subpopulation and proficiency range for their schoolwide focus. In 2008, the ITT was accessed through ARIS.

- Periodic Assessments: These diagnostic assessment tools aim to provide timely and detailed information to teachers about students' strengths and weaknesses, as well as their progress over time. These assessments were designed by educators to support 
student instruction. Assessment results are Web-based, available to every teacher, and can be disaggregated in any combination of formats. Assessment choices include Acuity (McGraw Hill) and/or Performance Series (Scantron). Students complete periodic assessments in ELA and math throughout the school year. There are no stakes attached to these assessment results for schools.

These tools are expected to support teams at different stages of their decision-making process. For example, the Quality Review and Progress Reports can inform the selection of the team's schoolwide focus, the ARIS and ITT tools can guide the selection of the target population, and the periodic assessments can support the monitoring of target population learning.

As this overview suggests, the 2007-08 school year was the critical implementation year for the Children First reforms as many of its components were available to school personnel for the first time. By design, the Inquiry Team process has been closely aligned and integrated with many of the new accountability tools available to schools. Even so, it is important to note that for most principals and teachers across the system, the 2007-08 school year was one of immense change and challenge. It included getting to know and learning to navigate the new environment of "empowerment"-its new structures, new organizations, new roles and relationships, new performance expectations, and new supports that surround and interact with schools. For some schools, this likely was energizing, but for others it may also have been overwhelming and at times confusing. The roll-out and implementation of the Inquiry Team process has been taking place within this broader context of restructuring. 


\section{Research Design and Methods}

The study focused on two key aspects of the Inquiry Team process - (1) how Inquiry Team members and principals have implemented the Inquiry Team process, and (2) how the external support structure of Senior Achievement Facilitators (SAFs) and Network Leaders from school support organizations have approached supporting the school-based Inquiry Teams.

The research questions that guided the study were created jointly by the Department's CFI leadership team and CPRE researchers. They focus on three key aspects of the Inquiry Team work: the implementation of the Inquiry Team process; team efforts to stimulate improvements in the conditions of teaching and learning in the school; and the nature and quality of support provided to Inquiry Teams.

\section{Inquiry Team Implementation}

1. How do Inquiry Teams spend their time when they meet?

2. Are Inquiry Teams using data to make decisions? What is the quality of data available, and how has it been used?

3. Do Inquiry Teams have a plan for their work?

4. What are Inquiry Teams doing differently as a result of CFI?

5. What role does the principal play in the work of the Inquiry Team?

6. What are the key obstacles to implementing the Inquiry Team process?

7. What does a successful implementation of the Inquiry Team process look like? Inquiry Team Work Schoolwide

1. How does the Inquiry Team work get shared with the school community? What role does the principal play in the process?

2. How is the Inquiry Team work perceived by non-Inquiry Team faculty?

3. To what extent do Inquiry Team members perceive themselves as leaders within the school?

4. How does the Inquiry Team work contribute to a school's ability to use data?

5. How does the role of the principal influence the work of the Inquiry Team and its ability to take the work schoolwide?

6. What are the important behaviors/practices that have an impact on changes in school culture?

Support for Inquiry Teams

1. How does the nature of support from the Network Team, SAF, and SSO affect implementation?

2. How is Inquiry Team support divided between SSO staff and the SAF?

3. Are there implementation patterns across SSOs?

\section{Study Sample}

Data supporting this qualitative study come from two sources. The first source includes one-day visits by CPRE researchers to a broad sample of 41 schools to interview the 
principal and each Inquiry Team member and to observe an Inquiry Team meeting. The second was interviews with external support staff associated with participating schools in this study, specifically Network Leaders and SAFs, and with the leadership of the affiliated School Support Organization.

Thirty-nine of the participating schools in this study comprise a stratified random sample of general education schools selected by CPRE in consultation with the CFI leadership team. The school sample was stratified by support provider, school network (with the goal of two or more networks per SSO), borough, school level, and school performance on the Progress Report and Quality Review, which served as a proxy for capacity. Two groups of schools were excluded: those who had switched SSO providers midyear and those scheduled to close by 2010. We also excluded New Visions schools because a different research study was already underway with those schools and dual participation would be burdensome. The 39 schools participating in this study were affiliated with six of the largest SSOs in the city. In 2007-08, over half of city schools were supported by the four Learning Support Organizations, about a third by the Empowerment Support Organization, and the remaining group of study schools by one of six Partnership Support Organizations. The five PSOs not represented in the study served relatively small numbers of schools. Since the number of schools affiliated with each SSO varied widely, the school sample for this study reflects this distribution.

By June, 2008, the final general education sample of schools for this study was 39. Of the initial 40 schools invited to participate, 35 responded positively. The four schools that declined participation were immediately replaced using another school with similar characteristics from the same school network. One school remained indecisive until the close of the school year and replacement was not possible. Principals who declined participation sited that their faculty felt overburdened by end-of-the-year schoolwork, or had already hosted a number of outside visitors associated with federal audits, Quality Reviews, or assessment piloting.

Table I displays key characteristics of the 39 general education schools and six SSOs in comparison with similarly affiliated schools within the New York City School System. As this table shows, this subsample has a slightly higher proportion of elementary schools, schools located in the Bronx, and those with a report card grade of "B" and a Quality Review score of "well-developed." In two cases, replacement schools were limited by network characteristics, namely the geographic location and size of member schools.

After the study was under way, we expanded the school sample to include special education schools at the request of the CFI leadership team. Existing study resources limited this sample to an additional two schools and affiliated external support staff (totaling five). These additional schools were purposefully selected by District 75 leadership because they were highly engaged in the Inquiry Team process. These schools were folded into the analysis with the core sample of 39 schools bringing the total number of participating schools in the study to 41 . To protect the confidentiality of these 
schools, throughout this report we include District 75 in general references to SSOs in this study.

Table 1. Characteristics of the Study's General Education School Sample Compared With the New York City School System.

\begin{tabular}{|c|c|c|c|c|}
\hline & \multicolumn{2}{|c|}{$\begin{array}{c}\text { School Sample } \\
(\mathrm{n}=39)\end{array}$} & \multicolumn{2}{|c|}{$\begin{array}{l}\text { New York City System } \\
\qquad(\mathrm{n}=1181)^{4}\end{array}$} \\
\hline & Number & Percent & Number & Percent \\
\hline \multicolumn{5}{|l|}{ Geographic Location } \\
\hline Brooklyn & 10 & $26 \%$ & 396 & $31 \%$ \\
\hline Bronx & 16 & $41 \%$ & 307 & $24 \%$ \\
\hline Manhattan & 8 & $21 \%$ & 246 & $19 \%$ \\
\hline Queens & 4 & $10 \%$ & 273 & $21 \%$ \\
\hline Staten Island & 1 & $3 \%$ & 59 & $5 \%$ \\
\hline \multicolumn{5}{|l|}{ School Level } \\
\hline High School & 6 & $15 \%$ & 278 & $22 \%$ \\
\hline Middle School & 7 & $18 \%$ & 302 & $24 \%$ \\
\hline $\mathrm{K}-8$ & 5 & $13 \%$ & 119 & $9 \%$ \\
\hline Elementary School & 21 & $54 \%$ & 582 & $45 \%$ \\
\hline \multicolumn{5}{|l|}{ Report Card Grade $^{1}$} \\
\hline 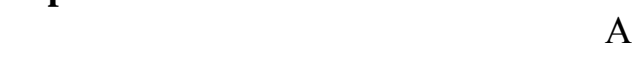 & 5 & $13 \%$ & 271 & $21 \%$ \\
\hline $\mathrm{B}$ & 18 & $46 \%$ & 452 & $35 \%$ \\
\hline $\mathrm{C}$ & 11 & $28 \%$ & 314 & $25 \%$ \\
\hline $\mathrm{D}$ & 3 & $8 \%$ & 100 & $8 \%$ \\
\hline $\mathrm{F}$ & 2 & $5 \%$ & 50 & $4 \%$ \\
\hline \multicolumn{5}{|l|}{ Quality Review Score $^{2}$} \\
\hline Proficient & 26 & $67 \%$ & 738 & $58 \%$ \\
\hline Well-Developed & 11 & $28 \%$ & 437 & $34 \%$ \\
\hline Undeveloped & 2 & $5 \%$ & 105 & $8 \%$ \\
\hline \multicolumn{5}{|l|}{$\begin{array}{l}\text { Experience With the } \\
\text { Inquiry Team Process }\end{array}$} \\
\hline First Year & 35 & $90 \%$ & NA & - \\
\hline Second Year ${ }^{3}$ & 4 & $10 \%$ & NA & - \\
\hline \multicolumn{5}{|l|}{$\begin{array}{l}\text { Affiliation With a School } \\
\text { Support Organization (SSO) }\end{array}$} \\
\hline Empowerment Support Organization & 12 & $31 \%$ & 430 & $36 \%$ \\
\hline $\begin{array}{r}\text { Integrated Curriculum and } \\
\text { Instruction-LSO }\end{array}$ & 9 & $23 \%$ & 351 & $29 \%$ \\
\hline Community-LSO & 6 & $15 \%$ & 155 & $13 \%$ \\
\hline Leadership-LSO & 4 & $10 \%$ & 100 & $9 \%$ \\
\hline Knowledge Network-LSO & 4 & $10 \%$ & 89 & $8 \%$ \\
\hline CEI-PEA PSO & 4 & $10 \%$ & 56 & $5 \%$ \\
\hline
\end{tabular}

${ }^{1} 94$ missing entries (7\%); ${ }^{2} 1$ missing entry; ${ }^{3}$ Schools in the Empowerment Zone, 2006-07 that piloted the Empowerment School Intensive (ESI) Process; ${ }^{4}$ Includes only NYC schools affiliated with six SSOs.

Schools were notified of their selection and encouraged to participate by the CFI Executive Director. As a follow-up, SAFs contacted their schools to provide information 
about the study and encourage schools to send to CPRE available dates for a spring site visit. About two-thirds of the school sample responded to this solicitation. The remaining schools received follow-up phone calls and faxes soliciting participation, first by CPRE and later by their SAF.

\section{Data Collection}

One-day site visits were made to the 41 study schools from May 1 to June 19, 2008. The study took an inclusive approach, seeking to interview all team members, not just a sample. In almost all schools, CPRE researchers were able to interview the principal and all Inquiry Team members, and to observe an Inquiry Team meeting. During the visit, researchers took notes using a laptop computer. School-level data collection instruments are available in Appendix A.

In two schools, the principal was not available for an interview (one did not return phone calls, another was on leave). In terms of Inquiry Team members, CPRE interviewed 204 of the 221 Inquiry Team members, or $93 \%$, in the 41 participating schools. Of the 18 members not interviewed, almost all were absent the day of the interview because of vacation, illness, or family needs (graduation, sick relative or child). A few were out on extended leave and therefore not scheduled. Only two teachers, both in the same school, declined participation. They reported being too busy or felt uncomfortable talking about the inquiry work with researchers.

One unexpected development was the large size of many of the Inquiry Teams in the study schools; the teams ranged from 3 to 12 members with an average of 6 members. To ensure all members had an opportunity to be interviewed, an additional CPRE researcher participated in site visits to schools with large teams. Finally, in 34 of the 41 schools, CPRE observed an Inquiry Team meeting. Team meetings ranged from 20 minutes to two hours in duration. Opportunities to visit team meetings were fewer in June because schools had either suspended Inquiry Team meetings or the team schedule was uncertain.

Table 2. Qualitative Data Supporting the Study by Level.

\begin{tabular}{|c|c|c|}
\hline Source and Type of Study Data & Targeted & Attained \\
\hline \multicolumn{3}{|l|}{$\underline{\text { School Level }}$} \\
\hline Principal Interviews & 41 & 39 \\
\hline Inquiry Team Member Interviews ${ }^{1}$ & 221 & 204 \\
\hline Inquiry Team Meeting Observations ${ }^{1}$ & 41 & 34 \\
\hline Total School Level: & 303 & 277 \\
\hline \multicolumn{3}{|l|}{ External Support Structure Level } \\
\hline School Support Organization (SSO) & 7 & 7 \\
\hline Leadership Interviews & & \\
\hline Network Leader Interviews & 18 & 18 \\
\hline Senior Achievement Facilitator (SAF) Interviews & 17 & 17 \\
\hline Total ESS Level: & 42 & 42 \\
\hline
\end{tabular}

\footnotetext{
${ }^{1}$ Based on the attained sample of 41 schools.
} 
Interviews with staff at the external-support structure level took place from May 19 to July 16, 2008. This extended period was a function of the study's school scheduling process, since SSO and SAF participation were determined by school participation, as well as by staff's busy schedules. All targeted interviews with SSO leadership, Network Leaders, and SAFs were completed. Most interviews were conducted by phone. As geography allowed, interviews with SSO leadership were conducted in person at the SSO office. All interview protocols for support staff are available in Appendix B.

\section{Data Analysis}

Data collection produced three independent data sets: school-based interviews, team observations, and support-level interviews. To support the integration and analysis of these data, we designed a qualitative data base using ATLAS.ti software. All data were coded using simple descriptive codes that were closely aligned with the interview and observation protocols and that were cross-referenced to the different data sets for analysis. We used a dynamic grouping feature of ATLAS.ti to sort data by important units (e.g., schools), roles (e.g., principals, Data Specialists, teacher Inquiry Team members), and relationships (e.g., network membership of schools, SAFs, and Network Leaders). Once all interview and observation data were coded, researchers developed a data summary profile for each school visited (see Appendix $C$ for the profile template). The profiles provided a synthesis of the views of team members and principals in a single data source. This was a critical step for exploring within-school variation. A third party performed quality checks for completeness on over $50 \%$ of the profiles. These profiles also were coded.

In the next stage a master data matrix was developed to track implementation across the 41 schools. As a companion to the profile narratives, the matrix further reduced the data set to a single visual display to facilitate the exploration of patterns and to test hypotheses about relationships among team features, actions, and outcomes as well as key contextual features. At this stage, some information about network characteristics and support staff working conditions and approaches to supporting the Inquiry Team process were linked with individual schools. In this stage, data were further reduced to yes/no responses (e.g., did the school have a clear change strategy?), or a new typology was generated to categorize school actions (e.g., roles of the principal as chair, contributing member, occasional participant, or nonmember). All interview and observational data and profile summaries were drawn upon in various combinations to complete the matrix. Researchers were responsible for writing brief memos and report sections drawing upon these different data sources and summaries. To safeguard study participants' identities, the female gender is used here to report data. 


\section{Report Overview}

The report is organized into eight sections. The first and second sections describe the initiative and the research design. The third describes the key features of the Inquiry Teams in study schools, including the size, composition of the teams, and the roles of the principal and Data Specialist. The fourth section explores team experiences implementing the different stages of the process, with attention to the team size and composition and the roles that principals and Data Specialists played in supporting the work. The next two sections describe how teams approached the different tasks of the Inquiry Team process and the quality of external support available to them. The final report sections review the work of the team to explore different notions of "accomplishment" for the first citywide implementation of the Inquiry Team process in 2007-08. This includes a discussion of the strengths and needs of the Inquiry Team process and some of the factors that may have facilitated or inhibited implementation. The final section presents the recommendations of the research team. 


\section{Key Features of School-Based Inquiry Teams}

Before examining school experiences with the Inquiry Team process, it is helpful to explore some of its key features. These features include the size, composition, and stability of Inquiry Teams, the roles taken by principals and Data Specialists in supporting the process, and the general dynamics of team work across the 41 study schools.

\section{Team Size, Composition, and Stability}

The members of a given team constitute the immediate resources available to engage in a deep reflective study of the learning problems in a given school. The critical resources that members bring to the Inquiry Team process are motivation, manpower, expertise, and social capital. The Department's guidance to principals suggested a composition of 3-6 members that would include the principal, Data Specialist, and classroom teachers (comprising a majority), with assistant principals, coaches, and other strong staff as possible additions. An emphasis was placed on selecting members with "strong leadership capacity."

Team Size. The Inquiry Teams formed across the 41 study schools reflected this guidance, with some schools making even greater investments (with their own funds) in the Inquiry Team process. Teams ranged in size from 3 to 12 members, with an average team size of 6 members. One assumption was that student enrollment would determine team size; however, this was not the case in the study schools. The four largest teams were found in what we considered small- ( $<400$ students $)$ and medium-sized schools (400-1,500 students). Among small schools, team size ranged from 3 to 8 members.

Team Composition. Team composition also varied widely across the 41 schools, with some important patterns to note. Principal membership on the team was not a given. In a quarter of the schools, the principal was not a formal member of team. As suggested, the school's Data Specialist was present on all but one team in our sample. Team members were drawn from three role sets in a given school: assistant principals, classroom teachers, and nonclassroom-based staff. Nonclassroom-based staff tended to include instructional coaches and staff developers, ELL/ESL teachers, academic intervention specialists (AIS) teachers or coordinators, and technology teachers, among others. In a few cases, guidance counselors, social workers, or consultants were asked to join the team, a group we categorized as "other." Nonclassroom-based staff comprised the largest group of members at $28 \%$, and teachers the second largest at $25 \%$ of members, while assistant principals represented $17 \%$ of members. A table detailing how team composition varied across the 41 schools is available in Appendix D.

Principals did take charge of composing their school's Inquiry Team, sometimes in consultation with their Data Specialist or assistant principals. In addition to posting the job announcements, principals publicized the formation of the team at different school 
meetings and some informally "encouraged" teachers and nonclassroom-based staff to apply. Principals reported taking a number of factors into consideration when composing the team. They sought expertise in content areas or with a special student population. They sought educators already in formal leadership roles, those with leadership potential, or those viewed as leaders among teachers. Some also strategically sought teachers who were responsible for the target population. Explained one principal, "We picked a $4^{\text {th }}$ and a $5^{\text {th }}$ grade teacher because we knew we would be focusing on one of those two groups, and we wanted the teachers' point of view." Some principals were unable to include teachers on their Inquiry Teams due to scheduling conflicts. Principals also wanted administrators who had supervisory authority associated with the focal content area or grade level of the teams. A secondary consideration for some principals was ensuring productive interpersonal dynamics, reflected in their concerns about how different personalities and work histories would interact within the team.

Team Member Reasons for Joining. Team member descriptions of how and why they joined their school's inquiry process reflected the strategies principals pursued in composing teams. They also revealed what attracted members to the opportunity and their expectations for the work.

Most teachers tended to respond positively to their selection to be on the Inquiry Team. Teachers thought they were selected for a number of reasons. These included their interest in or facility with data, work on a previous school-improvement initiative (e.g., curriculum revision or data use), current responsibility for or familiarity with target population students, ability to work well with students and adults, or held a reputation as "a strong teacher." Two new teachers thought participation on the team would provide them with feedback about their teaching, either directly from team members or through observing other teachers. Most teachers reported little understanding of the team's purpose before attending a meeting, beyond its emphasis on data use, helping a small group of students improve, and helping to improve the school. Many teachers viewed participation on the Inquiry Team process as an opportunity for professional development or personal growth and reflection. Two elementary teachers explained the attraction of the team:

I applied for my professional development. I wanted to do it. Children are my priority. If I can learn from this, then I wanted to see how it would help me to help children.

I think basically there was a posting, a discussion about it, and I had applied for the position. [CPRE: Why did you want to be on team?] I think [it was] going back to me being on the school leadership team and instructional team, they went well together. And I really try also to take a lot and apply it to my class and students, so I saw it as an opportunity.

A handful of teachers talked about the team presenting a leadership opportunity, or one fitting the leadership position they occupied as a senior teacher, or one that the principal 
emphasized in conversation. For example, one principal asked all grade leaders to serve on the team.

Team members who were nonclassroom-based staff tended to seek out membership on their own or were encouraged by the principal directly. Most thought they were selected for one of a number of reasons: experience with data use, content knowledge, knowledge of or experience with a special student population (e.g., special education, English language learners), currently serving on school cabinet or leadership among teachers, a close confidant of the principal, or the focus of the inquiry process aligned with their current duties. About half talked about their experience with or interest in data. Some felt it was important to be on the team since the team was focused on their content area of math or English. One literacy coach noted, "I wanted to be on the team. I have opinions about reading strategies." One thought it was a way to get to know what was happening in the school. Others said it "looked interesting" because they had heard about using data to consider how students were progressing. A few nonclassroom-based staff talked about membership as good preparation for future administrative positions. A few math coaches who participated on teams focused on ELA performance thought team membership presented an opportunity to prepare for the next year when math might be a focus. They also were generally attracted to the opportunity to look at student performance and "see the factors" that matter. A number of coaches were attracted to the idea of looking at student progress to "help benefit all students in the school," as noted by one literacy coach.

For most administrators, participation on the Inquiry Teams was expected by their principal because it touched upon their content area (e.g., math or organization/data) or a grade level (e.g., grades 3-5 or grade 9) they supervised, or it matched their previous work as a coach or teacher leader. One administrator was asked to serve formally as the principal's designee. The presence of administrators on the teams was even greater because a quarter of Data Specialists were assistant principals in study schools. In a few schools, all or part of the administrative staff was expected to participate. Administrators tended to share their principal's point of view and saw it as a "natural progression" or "fit." In a handful of cases, administrators volunteered to be on the team because they saw it as an opportunity to work with students, do research, or learn about what was happening in the school. One assistant principal saw it as a way to advance towards a principalship. Another saw it as good preparation in the event the Inquiry Team were to focus in the coming year on students in lower grades, which she was responsible for.

As this discussion suggests, some strategies for team selection brought resources and levers for supporting its work. The approach of many principals reflected their goal of ensuring that teams had the expertise, leadership capacity, and authority to complete the work. Some principals also strategically composed teams so that membership across school leadership bodies overlapped and that informal leaders among teachers were included. Looking across the 41 study schools, about 50\% included members with connections to other decision-making structures and processes in the schools. These included the school leadership team, school cabinet, Quality Review team, and/or grade level team leaders. These typically were administrators, but also included some coaches 
and senior teachers. In a few schools, union representatives were part of the team. This overlap in membership provided some political and organizational connections for the team to use when sharing their work with the broader school or when considering how best to take their newly acquired learning schoolwide for adoption. Also, some principals thought strategically about placing teachers on the team who were immediately responsible for students likely to be the focus of the Inquiry Team's work. At some schools, however, scheduling issues made it a challenge to include teachers on the team. A team member at one large high school explained:

We like to have the teacher [of the targeted students] here, but we can't always pull her out. We are on a split schedule here, so some of the team [members] are on early session and some are on late session, and we can't say we will just meet after school because of that.

Across the 41 study schools, about half had some or all of the target-population teachers as formal members of the Inquiry Team.

Variation in Team Composition. The composition of Inquiry Teams across the 41 study schools varied, but there were some common elements. Inquiry Team membership typically drew from three school-based roles: administrators, teachers, and nonclassroombased instructional staff. The few exceptions comprise an "other" category. Table 3 displays the variations in Inquiry Team composition across study schools by the mix of roles on a given team. (A table that lists each team composition is located in Appendix D). These team types focus on member roles only. According to principal and team member reflections on their decisions about team membership, key considerations for composing teams were focused on ensuring that teams had the expertise, authority, and leadership capacity to complete the work of the Inquiry Team.

In about a third of study schools, teams consisted of educators from the three role groups in equal proportions. These teams had a fairly balanced mixed of administrators, classroom teachers, and nonclassroom-based instructional staff. A handful of teams also reflected a diverse mix of roles, but were weighted towards one role group. For example, one team pulled together educators from a range of roles but was weighted with more teachers; it included one administrator, one math coach, and three teachers.

Another pattern that emerged was that of teams in which one or more role groups were not members of the team. This composition was found in $55 \%$ of study schools. For example, one team included an administrator, Data Specialist, two coaches, and a librarian, but no classroom teachers were included. Two schools had single-role teams, consisting of only teachers or only coaches. Team size appears unrelated to the team combinations, but was somewhat related to school level. In elementary schools, almost all Inquiry Teams, with the exception of one, had a composition that was diverse, with most balanced or weighted towards instructional roles with a higher proportion of teachers or coaches. Secondary schools tended to be composed of teams that did not draw across the three role groups - omitting coaches, administrators, or teachers as an entire group. 
Table 3. Variation in Team Composition by Member Role in the School ${ }^{1}$.

\begin{tabular}{|c|c|c|c|}
\hline Team Type & Description & $\begin{array}{c}\text { Percent } \\
(\mathrm{n}=41)\end{array}$ & $\begin{array}{c}\text { Number } \\
(\mathrm{n}=41)\end{array}$ \\
\hline Diverse-Balanced & $\begin{array}{l}\text { Team reflects balance of administrators, } \\
\text { classroom teachers, and nonclassroom-based } \\
\text { instructional staff. }\end{array}$ & $34 \%$ & 14 \\
\hline Diverse-With One & $\begin{array}{l}\text { Role Emphasized } \\
\text { Diverse team, but weighted towards } \\
\text { nonclassroom-based instructional staff }\end{array}$ & $12 \%$ & 3 \\
\hline & $\begin{array}{l}\text { Diverse team, but weighted towards } \\
\text { classroom teachers }\end{array}$ & & 2 \\
\hline & $\begin{array}{l}\text { Diverse team, but weighted towards } \\
\text { administrators }\end{array}$ & & 0 \\
\hline \multicolumn{2}{|c|}{ Mixed-With One Role Omitted } & $49 \%$ & 8 \\
\hline & Teams without classroom teachers & & 6 \\
\hline & $\begin{array}{l}\text { Teams without nonclassroom based } \\
\text { instructional staff }\end{array}$ & & 6 \\
\hline Single Role Teams & One role dominates & $5 \%$ & 2 \\
\hline
\end{tabular}

${ }^{1}$ Principals and Data Specialists as team members are not included in this analysis.

Team Stability. Most teams were stable in their size and composition throughout the school year. A few teams lost members due to medical or maternity leave. In a few cases, teams expanded to add needed expertise.

There were questions for some about how permeable team boundaries could and should be. Four teams had informal members, referred to as "the adjuncts" by one team, who sat in regularly with the team to offer advice. These participants reflected a variety of roles: social worker, guidance counselor, literacy coach, and ELL teachers. Teams that had little or no overlap with the target population teachers tended to raise this question about team membership in reflecting on their efforts to study the targeted students' needs, hear teacher views on their performance, and involve teachers in the change strategy design and implementation process. A number of teams informally expanded to include what we called "noncore members" with whom the team regularly conversed, but who were not formal members. There was ambiguity around expanding the team officially to include these new work groups or discussion groups, which were viewed as making major contributions to a team's inquiry, planning, and implementation. For some members, it raised the issue of how best to formally acknowledge the contributions made by these teachers, but also about compensating them for their time since formal team membership was funded. Said one team member: 
We should have had more teachers on the team. We should have had regular meetings with a group of teachers, an inquiry project for the teachers, who get together once a week, and pay them for their time.

This question about appropriate team membership raises a deeper issue about the flexibility of the teams to gain access to the expertise, authority, and leadership needed for the inquiry process. Generally, team composition appeared to match the general content area of focus-English, mathematics, or other - and the characteristics of the target population. However, there were 16 teams focused on English that had math teachers and/or coaches as members. In one instance, the Data Specialist - who also was a math coach - decided to participate only on an ad hoc basis. In these instances, this mismatch may have been because the team composition decision, which took place in August/September, preceded decisions about the team's choice of a schoolwide focus and target population, which took place by November.

\section{The Role of the Principal}

An overwhelming number of Inquiry Team members praised their principals for providing support, which varied according to teams' needs. Many principals were credited with getting teams "up and running," keeping members focused and on track to meet external deadlines from the network or SAF, and making sure members knew the "next steps." Even those principals whose contributions were more limited often were credited with keeping the teams focused, at the very least.

According to team members, principals provided teams with a range of organizational and material supports to facilitate the work at different stages. These contributions included the following:

- creating schedules to allow for common planning periods;

- providing coverage to enable members to complete work, attend meetings, or meet with teachers who had students from the target population in their classroom;

- providing professional development during the school day;

- reconfiguring classrooms;

- $\quad$ setting up before- or after-school programs;

- purchasing programs or materials; and

- providing necessary funding.

Principals varied in how they defined their relationship to, and role within, the Inquiry Teams. The formal relationships that principals formed with their teams fall into three broad categories: team chair, contributing member, and nonmember. Table 4 shows the distribution of these different principal roles across study schools. Within these three roles, principal participation ranged from being an active leader who was considered a "driving force" behind the Inquiry Team process to that of a general but distant supporter of the team's work. These roles emerged as principals talked about their involvement, but 
more importantly from the perspective of Inquiry Team members who were asked to reflect on their principals' contribution. Observations of the Inquiry Team provided opportunities to independently confirm the nature of principals' roles on the teams.

Across the 41 schools in this sample, the different roles that principals pursued with their Inquiry Teams does not appear associated with a particular level of schooling, the newness or size of the school, or the newness of the principal. Rather, it likely was driven by each principal's professional philosophy and interest in the Inquiry Team process.

Table 4. Variation in the Role of the Principal on the School Inquiry Team.

\begin{tabular}{|l|c|}
\hline \multicolumn{1}{|c|}{ Principal Role } & $\begin{array}{c}\text { Distribution Across } \\
\text { Study Schools } \\
\text { (n=41) }\end{array}$ \\
\hline Team Chair & $\mathbf{1 7 \%}$ \\
Heavily involved & 4 \\
Facilitator & 3 \\
Contributing Member & $\mathbf{5 6 \%}$ \\
Active participant/dominant voice & 6 \\
Equal footing with other members & 7 \\
"Stepped back" to build team capacity & 5 \\
Involved in start-up; participation tapered off & 4 \\
Seldom active & 1 \\
Nonmember & $\mathbf{2 7 \%}$ \\
Occasional participant/observer & 8 \\
Nonparticipant & 3 \\
\hline
\end{tabular}

Principal as Chair. Seven principals served as chairs of the Inquiry Team in their schools. Two different approaches to the role of chair were evident in study schools. In some cases, principals took a heavy-handed approach as chair, sometimes directing the team's work. These principals took an active role in defining tasks and drove the focus and pace of the process. Team members generally characterized them as "strong leaders" who were "hands-on" and attuned to every detail of the inquiry process. These principals held a strong vision for the team's work, and the chair's voice and opinions tended to dominate team meetings. This markedly proactive approach was seen as either an asset or a liability from the perspective of team members. For some, the principal's strong direction kept the team efficient and on task. Other members felt this style of leadership did not foster autonomy in the functioning of the team or creativity or openness.

In other cases, the principals used the chair positions as opportunities to facilitate the teams' work. These principals took a moderating role in the conversation, posing questions to the teams and letting members work through them in discussions. Principals in this role were viewed as collaborating members of their teams. Some members observed that these principals appeared more comfortable "giving up control" of the conversation or the definition of some tasks. In this role, principals sometimes offered their opinions last so as not to frame the conversation. 
Principal as Contributing Member. A majority of the principals were contributing members of the Inquiry Team, with another member chairing the meetings. In this role, principal contributions alongside their fellow team members ranged widely.

Principals who were actively involved as contributing members seemed to take one of two approaches. Some team members described their principals as respectful and encouraging of other team members' expertise, perspective, and opinions. They commented that they felt they were on an "equal footing" or shared "an equal partnership" with their principal during the meetings. Two teachers described what this looked like in practice and the meaning it had for them personally as a team member:

She was a real learner. Many times principals could take over, but she didn't do that. She wanted to be an equal partner. Most of the time we were all on the same playing field. She gave control. She sometimes facilitated. I'm proud to be a part of my school community because of it. [In this Inquiry Team] it was an equal partnership where we all supported each other. The principal knows all of our strengths and could bring it out.

She is a facilitator. She does not come in and give demands. We have a clear agenda. Everything is on the table. We know the guidelines. If she is concerned about something, she asks questions. She lets us brainstorm and she brings everything back together if we do go off on a tangent. She is very organized. And she is fair-listens to everyone's opinions. She wants to make sure all voices are heard. You feel like your opinion counts, you feel validated. When folks feel this way they feel like it's a group process, it's meaningful to you and everyone else. It becomes more authentic.

For many, this was professionally rewarding, as few in the building had opportunities to engage in ongoing substantive exchanges with their principals similar to those offered through the Inquiry Teams. In these cases, all members felt they had an equal voice in the decision-making process, and they described team meetings as being a democratic process. These principals contributed ideas and made suggestions to propel the process, but their voices did not overpower the teams. One member noted that her perception of the principal changed because of interactions through the Inquiry Team:

I saw the principal in a different light. She's an authority figure in the school, but in this team, she was easy-going. She wanted everyone's input. I liked seeing that side of her.

Members also valued principals who modeled what might be described as inquiryoriented thinking. These principals shared a strong curiosity about student learning needs, were open and even eager to explore a learning problem from multiple perspectives, and 
were comfortable acknowledging they did not know the answer. Some of these principals also had a "passion" and respect for data that team members picked up on.

We know we are never done. We come to something and then [the principal] sits back and asks questions and then we move forward. She makes us dig deeper. Even today...we had come to a place where we were talking about the TC instructional strategy, the actual boxes and what we were doing, and [the principal] asked questions about the kids who are still struggling, those four kids who are still not making it. She said "Yes, we have done that, but there are these four kids. They have made progress, and we can see that on the TC levels, but it is not showing up here [on the Acuity data], so dig deeper-what is going on?"

In contrast, some principals assumed a more dominant role, variously described by members as "a strong active participant," "guiding force," and "important voice." In this role, principals challenged team members to go further and deeper by asking probing questions to stimulate other ways of thinking about the process. Inquiry Team members generally regarded these principals as integral members of the team.

In several cases, the principal intentionally "stepped back" in order to build team capacity. One principal was careful to say that her position of authority in the school did not automatically make her leader of the team:

The best thing I did was to take a back seat. I think many times principals are filled with their own sense of self-importance. Each teacher brings creativity and [can] come at problems differently_put them together ... To me, that's building capacity.

This principal arrived late to meetings so that the team would have to start without her.

Still other principals took a more active role at the onset of the inquiry process, but their involvement tapered off or became intermittent as the year progressed. In many instances, these principals - like others in the study - were instrumental in assembling the teams and helping to determine the schoolwide focus and target populations, but were less involved later in the stages focused on identifying a change strategy, team outreach, and monitoring the target population. When asked about her role in the process, one principal commented,

I got [the inquiry process] started. But, I was often pulled in different directions ... I was mostly being a mirror [when I did attend], listening and reflecting ideas back to move the process forward.

In one case, the principal was active in forming the team but then was not involved for most of the remainder of the school year because of a busy schedule. Even so, the principal considered herself a formal member of the team. 
Principal as Nonmember. A quarter of the study's principals chose not to become formally or directly involved in the school's Inquiry Team. All but one of these principals were new, first-year principals.

For most, this meant that while they were not directly involved or responsible for particular team tasks, they did attend meetings occasionally. These principals acted as passive observers or onlookers during Inquiry Team meetings. These principals sat in on team meetings from time to time and gave necessary approval or suggestions throughout the process from the side. Members did not consider these principals particularly influential in the inquiry process. Principals said they took this posture to empower the teams to do the work. One principal explained that in an effort to cultivate a "sense of self-sufficiency" in members, she made a point not to attend every meeting or micromanage. Other principals also talked of the confidence they had in the teams and that they needed to attend meetings only occasionally to be "kept abreast of the team's work."

A few principals chose to have little or no direct interaction with the Inquiry Team in their school. These principals chose not to be formally involved in the team's work for a variety of reasons. One principal was retiring at the end of the year and "trusted" her team to do a good job. Rather than participate directly, one principal chose to stay updated through an assistant principal who was on the team.

\section{The Role of the Data Specialist}

This was the first year that schools had access to a Data Specialist in their schools. In almost all study schools, the Data Specialists were listed as formal members of the Inquiry Teams. Two schools had more than one Data Specialist on their teams. All Data Specialists were appointed by the principal. They felt they were selected based on their expertise and prior experience, or because their current job descriptions overlapped with the formal duties of the Data Specialist.

The role of the Data Specialist on the Inquiry Team was unique because these participants all held other positions and additional responsibilities in their schools. Table 5 shows the range of positions (a detailed list by team is available in Appendix D). About a quarter of all Data Specialists were assistant principals. Many others were concurrently in roles related to math and technology, such as coaches, teachers, and technology coordinators. A few Data Specialists were in roles related to special education. Principals reported selecting individuals who were comfortable with numbers, were familiar with data, or were "computer savvy."

Although Data Specialists wore many hats in the school, none reported experiencing any role conflict. If anything, they felt their position extended naturally either from their interests or their core role. Many found the Data Specialists duties demanding and felt the scope and quantity of work required to support not only the Inquiry Team but the school at large really constituted a full time position. One Data Specialist explained: 
The biggest obstacle is finding the time to get all that information from the different systems to create spreadsheets. We are not given enough time. I was not given enough time as a Data Specialist because I am full-time business manager and technology coordinator. They give us a little money to work before or after school and I feel the Data Specialist is a .5, if not a full-time position.

Table 5. Overlap Between Data Specialist Position and Other School Roles.

\begin{tabular}{|c|c|c|}
\hline School Roles & Responsibilities & $\begin{array}{l}\text { Percent of Study } \\
\text { Schools }(\mathrm{n}=39) \\
\end{array}$ \\
\hline Assistant Principals & $\begin{array}{l}\text { content areas, grade level, } \\
\text { test coordination, programming }\end{array}$ & $40 \%(15)$ \\
\hline Coaches/Staff Developers & math, literacy, AIS & $21 \%(8)$ \\
\hline Teachers & $\begin{array}{l}\text { special education, math, } \\
\text { social studies }\end{array}$ & $15 \%(6)$ \\
\hline Others & $\begin{array}{l}\text { teacher mentor, consultant, } \\
\text { unit coordinator }\end{array}$ & $13 \%(5)$ \\
\hline Test Coordinators & oversee test administration & $8 \%(3)$ \\
\hline Technology & computer, business & $5 \%(2)$ \\
\hline
\end{tabular}

Some Data Specialists who also held instructional positions, either as classroom teachers or coaches, shared nervously that their roles a Data Specialists sometimes took them away from their core jobs. They worried about how the principal or their supervisor would react at the end of the year when a review of their accomplishments reflected an emphasis on data and Inquiry Team duties.

Data Specialist Contributions to the Inquiry Team. Approximately one-third of the Data Specialists assumed the role of team chair or facilitator either voluntarily or by appointment. Many who assumed that role were nonclassroom-based staff while a few were administrators. Data Specialists who chaired the Inquiry Team were credited by members with keeping the team organized and on track by developing agendas, setting the tone for meetings, and ensuring that conversations flowed.

Data Specialists' involvement varied. Some - most notably the team chairs - were heavily involved throughout the entire process while others took an early lead, but their involvement tapered off or became intermittent as the year progressed. In other cases, their work was concentrated within a particular stage of the process. The majority of the Data Specialists were instrumental in determining the target population. For example, they often assembled spreadsheets to use in starting the process of selecting the target population and winnowing down criteria for selection. A large number were critically important in monitoring the target population via hard and soft data that they collected and analyzed from a variety of sources and presented to the Inquiry Team, often in the form of composite sheets or student profiles. A handful of Data Specialists took a lead 
role in developing the change strategies and instructional practices because of their particular backgrounds. For example, one Data Specialist was instrumental in developing her team's change strategy because it developed from her experience as a math coach. In another instance, a Data Specialist was particularly technologically inclined and became notably influential when the team developed a vocabulary tool that required a strong understanding of the Scantron Performance Series. Only a few Data Specialists appeared to take a leadership role in the team's outreach strategies. This often was in the form of professional development sessions on understanding assessment sources and use, such as with ARIS and Acuity.

While the Data Specialists' involvement varied, in most cases Inquiry Team members regarded them as having a solid grasp of data. Other commonalities in their positions, as noted by Data Specialists themselves and confirmed during Inquiry Team member interviews, included the overall management of gathering data for the team to explore and analyze. These included looking at and accessing data from Department information systems, most notably ARIS, Acuity, and Performance Series, and presenting findings at meetings.

Team Member Views of the Data Specialist. Inquiry Team members shared different views on the value of Data Specialists' contributions to their work. Members had more positive views of Data Specialists who were serving as chairs of the team, in addition to their other duties. In most instances, those Data Specialists were considered central to the Inquiry Team's work, described as "key players," "crucial to the process," "valued members," and the "glue that holds the team together." One assistant principal on that team asserted: "[The Data Specialist is] the best ... the chair ... the spokesperson ... the main person!"

In some cases, teams were ambivalent about the special contributions of the Data Specialist in relation to other team members. Those teams whose members were more versed in data collection and analysis seemed to rely less on the Data Specialist than those who had less experience.

\section{Team Dynamics}

For the most part, teams reported working well together. Over time their understanding of the purpose of the Inquiry Team process and the interconnectedness of specific tasks improved and members felt increasingly positive about their work. As the work of the teams gained momentum and clarity over the course of the year, teams reported their productivity and commitment to the process itself increased as well. During our end-ofyear site visits, a majority of team members seemed remarkably upbeat and optimistic about the future. Most team members were interested in serving on their teams the following school year. Some teams wanted to continue intact because they had come to work well together, which they considered an accomplishment and asset for the work of inquiry process. Team members typically spoke about their fellow teammates with 
respect and, at times, admiration because they felt they had contributed to their professional growth in large and small ways.

It's more than what I expected because we get the opportunity to have detailed, rich conversations about what can actually work and what is not working. Everyone bringing something to the table is valuable. The conversations are fulfilling. The work I did looking at data specifically was rewarding - helping to move children along was important as was the opportunity for interaction with colleagues. We all come together on different levels sharing ideas and thoughts about children and their data.

During team meetings, most members felt they were able to make a contribution to the process by looking at the task through their unique position in the school—as an administrator, classroom teacher, or staff developer. Two teachers summed up how all these different perspectives came together in their respective teams to advance the work of the Inquiry Team:

For the most part, the decisions we come to as a group ... It feels like everyone is sharing information. There is a mixture of role groups and experience levels on the team and knowledge levels. Several are not classroom teachers but they do coaching and lots of other things and have a lot of input to share.

We all took on roles on the team and that worked well. We all settled into these positions. [She] is the data expert and can look at numbers and report the key points to us. We as a group, the teachers say, "What would work in the classroom?" And I say, "How will technology help?" And the principal says, "How will we implement?"

Work Distribution. The inquiry process itself required teams to move between micro and macro views of the school, shifting from the learning of individual children in a given classroom to a building-wide view. Almost all members felt their content expertise and experience working with particular student populations were valued during the process. At different stages of the process, some members were relied upon more than others to push the work forward. This meant that while all members were engaged throughout the process, some members moved to the forefront on particular tasks, team conversations, and drafting work products for team review.

Many members reported, sometimes emphatically, that the team was a collaborative effort and everyone contributed equally. Yet within this commitment to share the work was a respect for those well positioned to conceptualize, draft, or lead individual tasks. In most schools, the flow of teamwork reflected an evolving distribution of the tasks across team members based on best fit and availability. Typically, the Data Specialist was critical throughout, most notably in helping the team access, interpret, and compare data results and assessment choices for determining a focal group of students and diagnosing their learning needs and monitoring student growth. Coaches often took the lead in 
identifying instructional strategies for their target populations with teachers helping to refine those strategies and implement them in the classroom. Administration contributed organizational and material resources to the teams, signaled the importance of the work to the larger school community, and helped craft an implementation strategy.

Teams that were diverse and balanced in membership across role groups reported a shifting and sharing of the Inquiry Team work among teammates. This was not the case for some teams, where the work of the team was concentrated in one or two members. In some cases this happened by default; teams were small, or only one person had the expertise or the availability (and will) to do the work. For those who shifted into these roles, the leadership opportunity and responsibility were both fulfilling and overwhelming. Here is one literacy coach's experience serving on a single-role team, in which no assistant principals and classroom teachers were participating.

I didn't have any expectations [about joining the team], but I feel I am taking on a larger role than I expected. I thought it would be a team effort and we would assign roles, and that has happened. Because we all did classroom walk-throughs and were assigned different teachers for that. But I guess because I am the Literacy Coach, for this part [of the work] I have taken on, in terms of learning the strategy and helping the team learn the strategy and then turn-keying that to teachers who will then turnkey to their students, I am taking on a larger role than I thought I would. But that is OK, I am kind of enjoying it.

This experience reflects an extreme case within this sample of schools but offers a useful illustration of how team composition can influence the distribution of labor and availability of expertise to team members.

Teacher Contributions and Constraints. As teams progressed through the process, the contribution of classroom teachers as members in the Inquiry Team process increased. They represented an important perspective for administrators to hear, offering a connection to what was happening in classrooms, refining chosen instructional strategies, and helping to implement and model their team's change strategies with target population students. Here two administrators share their experiences:

Conversation with teachers is valuable, that you have time to sit and talk about children and how they progress and instruction. All of the administration is on the team and we are all looking at the data togetherit's a new skill for everyone. It's a great learning process.

[The Inquiry Team process] is worthwhile for me, because listening to the teachers and having the opportunity to ask why and what do you think and then having the data and looking at it and going into the classroom. When I visited, I looked at the targeted children a little more. You become more aware-it's an awareness level. 
When the teachers responsible for the target population students were not on the team, some teacher members became an important bridge for reaching out to them, peer to peer, to inform them of the Inquiry Team work and solicit their ideas and involvement. One testing coordinator characterized teachers in this role as "emissaries" of the team's work.

Some teachers felt overloaded by the work demands of team membership and their core responsibility as a classroom teacher to 20-30 children on a daily basis. One teacher described the tension between the two and the challenge to find the right balance.

I was not concerned about the work [initially], which intrigued me, but whether I would be able to do the work in a good way... My issue now is time management. We kind of thought I would be able to do it, but it is difficult to fit into my normal responsibilities as a teacher, a grade leader, and other projects I am doing, and this as well. [CPRE: What kind of demands does the Inquiry Team make on your time?] There [are] some reading assignments to be done, some analyzing of student work. The good part for me is that I am working with my students in my room, so it makes it a little easier. But at the same time, I am responsible for teaching 22 kids, not just 3 ... so it is demanding on personal time.

Teachers reported using their lunch or preparation period for team meetings or tasks. Also, the unexpected needs of students and parents made teacher attendance sporadic in few schools. This tension was not lost on their teammates, who tried to buffer the pressure by taking on some additional work, particularly administrators, coaches, and Data Specialists. Many teachers want to continue to participate and teams have found other teachers interested in joining next year.

Team Meeting Observations. The opportunity to sit in on Inquiry Team meetings offered brief windows into how teams were organizing their work. CPRE researchers were able to sit in on 34 team meetings to observe how well teams were functioning in five areas:

1. The extent to which the observed discussion reflected an understanding of the Inquiry Team process, in general, and/or the current tasks, in particular;

2. The professional tone of the meeting;

3. The extent to which there was an established routine to the meeting and roles were well-established;

4. The level of participation across members; and,

5. The quality of team member relationships.

Each area was rated on a scale of 1 to 5, with 25 as the highest rating for an observed team meeting.

Team meetings receiving higher scores of between 21 and 25 appeared strongly engaged in the inquiry process. These high-functioning teams accounted for $64 \%$ of observed meetings. These members exhibited a strong sense of the Inquiry Team's purpose and its connections to other aspects of the school such as instructional practices and school 
structure. Additionally, these observed team meetings typically were characterized by broad participation among all team members, however small or large the team, and the meeting structures reflected established routines and flowed smoothly and efficiently. In contrast, team meetings with lower scores of between 11 and 15 were characterized by some weak areas. These lower-functioning teams accounted for $18 \%$ of the observed meetings. During these team meetings, members appeared less engaged and the conversation reflected a compliance approach and surface understanding of the overall process and its different parts. Additionally, these team discussions tended to be dominated by a few members and to lack coherence and the meeting lacked a clear routine. The remaining $18 \%$ of observed teams were moderate functioning, scoring between 16 and 20.

Table 6. Team Meeting Observation Ratings by Principal Role.

\begin{tabular}{|l|c|c|c|}
\hline Principal Roles in Inquiry Teams & $\begin{array}{c}\text { Average } \\
\text { Score } \\
\text { (up to 25) }\end{array}$ & Range & $\begin{array}{c}\text { Percent Scoring } \\
\text { High: 21-25 points } \\
\text { (n=22) }\end{array}$ \\
\hline $\begin{array}{l}\text { All Team Meeting Observations } \\
\text { (N=34) }\end{array}$ & 21 & $12-25$ & $64 \%$ \\
$\begin{array}{l}\text { Principal } \\
\text { Chair-Heavy Handed } \\
(\mathrm{n}=4)\end{array}$ & 15 & $14-17$ & $\begin{array}{c}0 \% \\
(\mathrm{n}=0)\end{array}$ \\
$\begin{array}{l}\text { Chair-Facilitator }{ }^{1} \\
(\mathrm{n}=3)\end{array}$ & $\mathrm{NA}$ & $\mathrm{NA}$ & $\mathrm{NA}$ \\
$\begin{array}{l}\text { Contributing Member } \\
(\mathrm{n}=19)\end{array}$ & 23 & $13-25$ & $\begin{array}{c}41 \% \\
(\mathrm{n}=14) \\
21 \% \\
\text { Nonmember }\end{array}$ \\
$(\mathrm{n}=8)$
\end{tabular}

${ }^{1}$ Data were incomplete for teams in which the Principal was a chair-facilitator.

As mentioned, of the 34 team meetings we observed, about two-thirds appeared high functioning, earning a score between 21 and 25 . In fact, $30 \%$ of those team meetings earned the highest rating of 25 . The role of the principals in these team meetings may have contributed to how well they functioned. In particular, team discussions in which the principal was the chair and used that role in a heavy-handed manner to direct and oversee the team's work reflected lower scores, with an average of 15 . In contrast, team discussions in which the principal was a contributing member tended to be high functioning, with an average score of 23 , and reflected $41 \%$ of the higher scoring meetings that we observed. Data Specialists also have taken on leadership roles, formally and informally, for the Inquiry Teams. In meetings where the Data Specialist was the chair, the average rating was fairly high at 21 points. Table 6 displays the overall scores for team observations and a breakdown by principal role. 


\section{How Teams Approached the Inquiry Process}

In this section we examine how teams approached the many key stages and tasks of the Inquiry Team process presented earlier in Figure 1. This description includes what teams were able to accomplish and the types of challenges they encountered.

\section{The Pace and Completion of the Inquiry Team Work}

By the time of the CPRE site visit in late spring of 2008, a vast majority of the study schools had completed a full implementation cycle of the Inquiry Team process. There was some variation in the quality of implementation, but a major accomplishment was that almost all teams had carried out the key aspects of the work, with many already eagerly planning for the 2008-09 school year.

For a number of team members, the start-up of the Inquiry Team process was slow, which generated some uncertainty and frustration during the early fall. Initially, team members reported feeling confused about the purpose of the work. One teacher's comment is highly representative in this regard: "Honestly, I had no idea what to expect. For the most part, we were all feeling our way through." For most, the job announcement was the only description of the process available. Many members felt left on their own to figure out the specific tasks, particularly at the beginning of the year, and some reported feeling a little uncertain throughout the year as to whether they were moving at the right pace: "We never were sure if we were behind others. Are we where we should be?" Another teacher's reflection on her team's pace and need for guidance also highlighted how other Department activities sometimes competed with the Inquiry Team work for attention within the school:

We got off to a late start. There was some confusion in terms of how specific our goal should be. We were too general at first. By the time we focused ourselves, it was later in year. Also, there was an early Quality Review this year which threw us off so we focused on the Quality Review. ... Our meetings began in late fall. We didn't consolidate our goal [for the target population students] until February.

This team's timeline was on the extreme end of the spectrum-efforts to focus the team's work extended into the middle of the school year. The effect of the late start for most was that it shortened the time available to work through later stages of the process. For some teams who were ready to share their learnings at the end of the year, this presented a lessthan-ideal situation as the impact of end-of-the-year training for teachers would be weak and short-lived. These teams went ahead and planned their professional development sessions but decided to implement them at the beginning of the next school year.

In most cases, schools at the other extreme-those with teams that started the work early in the year-had experience with the Empowerment School Intensive process the 
previous year, a forerunner to the Inquiry Team process. Many of these schools had planned for the Inquiry Team work in June, 2007 by selecting a schoolwide focus and subpopulation and soliciting team membership. These schools hit the ground running at the start of the school year.

The confusion experienced by many of the first-year Inquiry Teams may reflect the typical start-up issues associated with rolling out any new initiative compounded by the general confusion associated with the beginning of the school year. Additionally, the start-up period was extended for many by the need for basic training in the new tools such as ARIS, the Progress Report, and Quality Review, which stretched into the late fall for most teams. Jump-starting this process at an individual school level were visits from Senior Achievement Facilitators (SAFs) who met with teams to provide guidance and answer questions. In some cases, SAFs and some Network Leaders reported checking in early with principals to make sure the teams were being established. Many teams did not gain much clarity about the Inquiry Team process until November.

What is certain is that by the close of the year, most team members felt they had gained a stronger understanding of the purpose and key features of the Inquiry Team process simply by having worked through it first hand. One factor that helped to push some team members forward through the initial confusion was their sense of commitment to the target population students they had selected. One teacher's response reflects the obligation members felt about making a difference for these selected students who members had come to know personally over five months or more.

Looking at this year, we started with a lot of uncertainty and got to the point where we realized these 15 kids are of huge importance to us and we have to get them moving. Then what we do with them we hope we can do with other kids. Once that became clear, the rest became easy, with that clarity. [CPRE: What became clear?] The purpose really became clear. In the beginning, although we knew we were picking kids and trying to move them, but just not that sense of, here is where we are and here is where we are going. So it will be better next year.

For this teacher, like so many, connections to the larger school and an understanding of how all the tasks fit together became more clear by the late spring when the CPRE site visits began. By the end of the year, most team members reported feeling exhausted but also proud of the growth they were seeing in their targeted students and the intensive study they had conducted in their school. Only a few teams that had struggled to get started on and off during the year were ambivalent about their accomplishments and the continuation of the process, but these instances were the exception in our sample.

\section{Identifying a Schoolwide Focus}

The first substantive task for teams was to identify a content area and a subpopulation of students within that area who were struggling. In a majority of cases the process was 
sequential, with the content area identified first followed by the subpopulation of students. But in others these decision points were simultaneous or even in reverse order. In the later case, the process began with what was considered "common knowledge" at their schools, specifically that a particular subpopulation was struggling and in need of attention, typically English Language Learners (ELLs).

Selecting a Content Area. All schools identified a schoolwide focus to guide their team's work. The vast majority of study schools - just under 75\% — chose English Language Arts as their content area of focus. Math was an area of focus for $17 \%$, with the remaining schools focused on other areas such as social studies, credit recovery, and communication skills. For many schools, the chosen focus reflected a significant area of low performance, what many considered to be an area in which a majority of students in the school were struggling.

In most study schools, the selection of the schoolwide focus was left to the newly established Inquiry Teams. Most reported this was a collaborative process. In about a quarter of schools, the principal selected the particular content area of focus. In some cases, the principal presented the chosen content area for review by the new Inquiry Team. In one case, the principal sought approval for the selection from the school cabinet. Principals' involvement in these decisions appeared connected to the strength of their opinions about the priority needs of their schools and therefore reflected their efforts to focus the teams on those areas immediately. It may also be connected to the team selection process which started before school opened in some cases. By determining a particular content area, which also suggested a grade level or student population, some principals were able to ensure a good fit between team composition and the substantive focus of the team's work.

In a number of cases, typically in those schools who selected mathematics or other content areas as their focus, the rationale for the focus was highly contextualized to the school. Compared with the rationales that undergirded the choice of the ELA content area, which typically seemed straightforward, these choices were complex, based on existing programs, prior improvement history in a given content area, and the capacity of teachers within particular disciplines to fully engage in the Inquiry Team process. Consider the following examples drawn from team member descriptions.

- A glaring area of weakness at School 37 was science, but math was also weak. The principal considered the strengths of the math and science department faculty and found the administrative leadership for math was stronger and the math teachers more cooperative and cohesive than was true for the science department. For these reasons, it was hoped the math department would be more receptive to the Inquiry Team process. Also, the composition of the Inquiry Team already was weighted with math educators. So the team, under advisement of the principal, decided to target math.

- The team at School 14 selected math because other programs to improve literacy skills already existed in the building. They felt the process presented an opportunity to focus on math. Many felt math skills would be easier to 
address as a subject because many of the school's students were not from the United States. They felt math presented a common language for all and unifying focus for teachers. The team also wanted to take advantage of a new math program adopted by the school. The team chose a grade that enabled them to track students' progress for two or more years and the capacity to review data from previous grades.

These examples highlight the strategic efforts made by principals and some teams to fill gaps in the instructional support being provided for particular student populations in their buildings, to strengthen initiatives already under way, or to strategically leverage new or available resources associated with a specific content area and/or subpopulation of students. These examples also demonstrate how the Inquiry Team work often was strategically situated within a school from the beginning of the process.

A few schools selected the subpopulation first-ELL or special education students in most cases - and then chose ELA as a focus area. In a few cases, the expertise of Inquiry Team members was an important factor.

About $15 \%$ of the schools chose their content area because it involved skills team members felt could be leveraged for student improvement across the curriculum. In most cases it was ELA. Some middle and high school teams also saw social studies as a way to focus on inferencing skills that they believed would transfer easily to other subjects and therefore benefit all students. One school initially wanted to focus the Inquiry Team work on science and social studies, but shifted to English when their data analysis suggested that reading comprehension was a common core challenge for the bottom third. One school with a strong multilingual immigrant student population thought focusing on mathematics would enable the team to be of high relevance to most students in the school. Finally, a few schools based their decision on the citywide initiative in ELA.

Almost all schools used assessment data to guide their selection of the content-area focus, and most based their decision on the previous year's state test scores. For example, almost half of the teams compared state assessment scores on math and English, and then selected the area in which students were performing the lowest, on average. Although state assessments scores were the main source of data guiding the selection process, other sources include Progress Reports, Report Cards and Quality Reviews, ARIS, SAT scores, Performance Series assessments, student grades, surveys of student interest, and teacher recommendations. Most teams conducted the data analysis themselves and generally relied on state data from the previous year. One school reported working with a city Data Specialist to examine the school's state assessment results over three years.

In three cases, teams were asked by their SAF to change their focus because it was too broad. These teams either had not yet identified a subskill focus or were focused on two content areas. Two teams reported being asked to narrow their chosen focus from two areas to one. One school complied, but another secretly kept the second focus throughout the year, an action they felt was justified because it was important and of interest to team members. This determination speaks to the strong opinions held by some members about 
addressing the needs of overlooked student populations in the school and their personal connections to those populations.

Focusing on a Subpopulation of Students. Next, new Inquiry Teams were expected to determine a subpopulation of students who were struggling in the chosen content area. To help focus schools on closing the achievement gap, the department encouraged teams to select a subpopulation from the lowest performing students, specifically the bottom third. The subpopulation approach was intended to help teams further focus their work on manageable groups of students.

Decisions regarding the subpopulation selection were largely left to the newly formed Inquiry Teams. Team members described this process as relatively collaborative. A few members spoke of having strong attachments to particular groups of students and were disappointed they were not selected. But the initial guidance from the Department, and later SAFs, that suggested teams consider the lowest-performing students worked to keep them focused on closing the achievement gap.

The student subpopulations that teams selected varied widely. Three-quarters identified subpopulations based on state assessment scores. Often, this was the bottom third of students on a specific state assessment, but in some cases, it was also a specific focus on students who scored $2 \mathrm{~s}$ or $3 \mathrm{~s}$ on the state exam. A few schools used Acuity or Scantron scores as well. About a fifth of the schools chose to focus on a special student population, ELL students typically, while a handful of teams focused on special education students.

Student subpopulations were further reduced in size by considering grade level. While less than half of teams focused on a single grade, a majority identified subpopulations that spanned multiple grades, ranging from two grade levels to six. This choice was not associated with school level or team composition. Elementary schools tended to focus on grade four students to access to state assessment data and to track student growth within the school. A few middle schools focused on $7^{\text {th }}$ grade students for the same reason.

The reasons team members gave for selecting a particular subpopulation also varied. Teams tended to select student groups that gave them access to strong data sets to support analysis, were most likely to affect the school's AYP or progress report grade, or would be easier to help improve. Another frequently cited reason for selection was that the student group in question was most at risk of failing, usually on a state assessment. A similar number of teams selected a population that could be monitored over multiple years. A few teams focused on a subpopulation because it represented a large percentage of the school enrollment - teams usually gave this reason when selecting ELL students as the subpopulation.

There is some evidence that at this initial stage of the process, some teams were already thinking strategically about how their choice of content area and subpopulation would affect other aspects of the Inquiry Team process. A few teams considered teacher capacity to benefit from the inquiry process, both in helping to implement instructional change strategies and willingness to use the insights gained from the process. A few 
teams sought a student group that was generalizable across their schools so the strategies they developed would be useful with other students and of interest to other teachers. For instance, teams might select one or two student groups that represented $50 \%$ or more of those enrolled, select a cross-section of prominent student subgroups so the subpopulation was truly representative of the school, or include students from many or all grade levels. Other teams selected students at grade levels that could be tracked for 1-2 years.

Reflecting on their approach to identifying a content area and a subpopulation, many team members felt they could improve the process by simply starting earlier in the school year. One team that focused on an ELA content area hoped that by starting earlier in the year, they could use the mid-year state ELA assessment to measure student learning.

One of the issues was that the deadline for identifying target population was mid-November [but] the ELA exam is in January. And we are supposed to measure a year's worth of growth. We want to see it reflected in the ELA score because that is what you use to identify the bottom third to begin with. So if you don't identify the students until November, are you seeing progress by January? That is kind of a problem in terms of the scheduled date for testing. So maybe now that the initiative is not brand new, if we know it will continue, maybe choosing them earlier would be better to get started in September. Part of the issue is not getting scores back in a timely manner, but we would like to identify the students sooner and get the work going sooner so you can see the year's worth of growth.

Some members also recognized a need to narrow their focus even further. These were teams that had stretched the selected subpopulation across many grades or had opted for two goals, rather than one, which doubled their work. One math coach's views reflected these themes.

[CPRE: How would you improve the process?] Narrowing it down for next year. [This year] we were ambitious on what we wanted to do. Some maybe want to focus on one grade instead of two grades, want to focus on a few skills and few standards. So narrow down our goals is one change. I think we have been fabulous, doing a great job even though it was not clear what we had to do. As for internally, things weren't clear to us even after going to meetings-but it got clearer as we went along.

A few ambitious teams were actually planning next year's schoolwide focus before the school year ended so they could "start the next year running." This selection was sometimes associated with the current target population of students, which some schools wanted to continue to track, but also often reflected considerations about how to expand the process to address other areas of need at the school. 


\section{Selecting a Target Population}

Once teams settled on a schoolwide focus, they were faced with the task of narrowing down the pool to a smaller set of target population students to be included in the Inquiry Team work. The size of the target populations that teams ultimately selected varied widely, from 12 to 38 students. The majority of teams, about $54 \%$, focused on groups of 15-16 students. One school selected a sizable target population to allow for some attrition because the chosen subgroup of students, here ELLs, had a high transfer rate at the school. Teams that chose two different schoolwide foci tended to have larger target populations.

Teams used a variety of strategies to select their target populations; but most teams narrowed their initial pools of students based on a combination of objective and subjective characteristics. Most frequently, this included targeting students with relatively high attendance rates, narrowing the range of test scores, taking into account teacher and team member opinions about which students needed extra help or had the greatest potential to improve, and excluding students with serious behavior problems. In a few cases, classroom teachers on the Inquiry Team who taught the subpopulation students hand-picked a set of students for the target population; each had to justify their selection to the team. In two schools, the target population was based on student enrollment in an existing school-based program.

About two-thirds of the teams identified a more specific subskill on which to focus their work with the target population. The most common subskill was reading comprehension. Others included vocabulary development and inferencing which often was selected by teams with a social-studies focus. Other examples of subskills identified by teams were the use of context clues, use of graphic organizers, identifying main and supporting ideas, differentiating between relevant and non-relevant information, and fractions. A few teams identified more than one subskill to focus on. Another handful of schools decided to identify a skill and a subskill for each individual student, rather than for the group or the entire target population. In these cases, teams used data from the Acuity, Performance Series, and state assessments. Six of the teams did not identify any specific skill within the content area of focus, but rather focused generally on the content area or on test preparation.

Teams that identified a specific subskill typically used results from student assessments, usually state assessment data, to identify a common area of weakness within the target population. Most often this was based on an item analysis of the assessment questions. State assessments were the most frequently used data sources, but other sources included Acuity, Performance Series, Teachers College running records, ECLAS-2, DRA, NYSLAT and low-inference observations. In one case, a team administered a selfdesigned survey to students.

The schools that did not use assessment data to identify a subskill typically relied upon other indicators. These included students' prior placement in an existing program, reviews of state standards to select subskills that were perceived as foundational for 
future student work in a given area, or intuition or personal experience with students. Other reasons given for selecting particular subskills appeared driven by larger concerns and opportunities, specifically that a significant portion of an upcoming assessment measured the skill or a conviction that all teachers working with the target population were capable of implementing a change strategy that addressed the specific subskill.

As with their selection of a schoolwide focus, some schools selected target populations to help leverage change schoolwide. In this regard, slightly over $20 \%$ of the teams selected target populations that were representative of their larger school populations. A few schools ruled out the inclusion of special education and ELL students on the grounds that excluding these types of students would enable them to generalize their work to the larger school population. These teams felt that the challenges facing special education and ELL students were unique. In the same way, a handful of teams thought about the transferability of the selected subskill within the wider school community. These teams believed the chosen subskill was a stepping stone to learning specific content or other skills in a given content area. This rationale was used by teams that focused on basic fractions, vocabulary development, and for some, inference making.

\section{Developing and Implementing Change Strategies}

Developing and implementing change strategies to improve the performance of the target population was one of the most challenging tasks faced by Inquiry Teams. Identifying a focus and a target population was relatively straightforward; teams examined available student performance data and followed the department's guidelines of focusing on the lowest-performing third of students. Once the schoolwide focus, specific subskills, and target population were identified, however, many team members reported a feeling of "where do we begin" in terms of identifying strategies to improve student performance.

Teams varied in the amount of time, energy, and research devoted to what was for some a daunting task. About two-thirds of the teams in the study sample had developed and implemented a clearly articulated change strategy at the time of the research visit. Others had simply identified a target population and a subskill and then asked teachers to focus on that skill-without providing them with a specific change strategy. Some teams did not discuss specific strategies because the target students were already enrolled in a number of programs that would provide help with the needed skills. In these cases, the programs became the de facto change strategy.

Somewhat surprisingly, most teams did not fully utilize their networks or SAFs at this step; they did not ask for support or seek assistance in developing change strategies. Outside staff had been helpful in launching the work of many teams, but we heard few reports about support being provided during the selection or development of change strategies. Instead, Inquiry Teams relied primarily on internal expertise and resources to identify how they might address students' learning needs. 
Across this sample of schools, the change strategies advanced by the teams were aligned with the content areas they had identified. The change strategies adopted varied on a number of dimensions: the number and complexity of change strategies identified, the duration of the change strategy, the number of teachers involved in implementing the strategy, and the number of students affected by the change strategy. These aspects are discussed below.

Breadth of Search Process. In developing a change strategy, roughly half of the teams in the study sample took the highly pragmatic approach of calling upon the expertise and resources of the team members themselves. Team members were often selected because principals felt they were able to meet the needs of the target population, so the appropriate expertise was often available on the team. For instance, schools focusing on reading comprehension likely had literacy coaches and teachers from the appropriate grade levels serving on their teams. An Inquiry Team that had targeted reading fluency pooled the expertise of the literacy coach (who was on the team) and Reading Recovery teachers in the school, knowing that these teachers did a great deal of work with fluency. From the options presented by these teachers, the team chose a timed reading strategy because they felt this was something that team members could implement. The literacy coach explained:

When we began pulling up instructional practices ... I could suggest fluency activities. We tapped the Reading Recovery teachers because they do a lot of work with fluency in the beginning readers, and some of our older students are reading at that level. Their strategies are very concrete and straightforward in fluency, so we tried to get presentations and dialogue going between Reading Recovery and [Inquiry Team] members who are not used to reading. We had a menu of strategies, but then we picked one that we would all use, and that was timed reading.

A smaller number of teams conducted broader searches that examined a wide array of resources including expertise in other schools, products available from commercial vendors, education trade magazines, or research sources. Even in this subsample, however, the search and/or development strategy of most teams was not extensive, and still drew heavily on the school-based expertise of classroom teachers and coaches on the Inquiry Team or invited teacher collaborators. The press for time likely contributed to these abbreviated searches. For instance, an elementary school team that was focusing on the subskill of using context clues to understand text decided to implement a strategy suggested to them by the principal's mentor, based on an article in a professional journal. The literacy coach made contact with the authors of the article to discuss the strategy, and then modeled the strategy at an Inquiry Team meeting. The team then decided to adopt the strategy. A middle school team simply identified existing programs in the school that focused on the targeted subskill (drawing conclusions) and made sure that the target population worked in these programs during regular classroom time and in any afterschool or intervention programs in which the students already participated. 
In a few cases, teams engaged in a more intensive research and development process. A classroom teacher who served on the team at her school described the process used by her team to develop a change strategy to help $4^{\text {th }}$ grade ELA students use context clues to learn social studies and science vocabulary:

Our first cycle [of the change strategy] was to determine what vocabulary words to use. We chose social studies words that would go across the curriculum, not just for social studies, but words they would see on the ELA exam or in other subjects ... We chose vocabulary words with the help of the literacy coach and the social studies pullout teacher at the time. We picked out 15 words. From there, we had to come up with our pre-test, which was a lot [of work]. It was just doing it the first time, it became a variable-if they read a longer passage, is that another reason they don't understand vocabulary or is it just that they can't read the word? We learned from that what worked and didn't, and we created a pretest using those vocabulary words ...

After the pretest, we met as a group and looked at the results and did a little noticing - was it the longer passages they had trouble with, was it the more challenging words with more syllables? From there, we developed our mini-lessons to do with the students. We separated the students into three groups of low, medium, high, and we have three teachers who work with them during 50 minutes and I am one of them. We all do the same mini-lessons, materials, and strategies with all three groups. We created those with the literacy coach; her, myself, and another team member met and created these mini-lessons, and then the actual texts. We went into different textbooks and on-line resources and things in the Teacher Center and compiled them and created texts that we would use for mini-lessons.

That was done over a couple of days. The literacy coach pulled ideas pretty quickly and she would send them to me and I would type them up. So we developed mini-lessons for the four-week cycle. It turned out to be only three lessons. Right now we are doing science, so on Tuesday we do a concept map with the vocabulary words. On Wednesday, we do an experiment and the students are using the words in the experiments. On Thursday they read a passage and we discuss it and they answer questions. So it is those three lessons, which we do over and over but with different words. We will do five words this week with those three lessons and five the next week and so on. That generally only takes three weeks, but we do the pre- and post-test.

This teacher enjoyed working on the Inquiry Team and reported that she had learned a great deal about working with ELL students. She noted, however, that the process of developing a change strategy consumed a lot of time and energy, and wondered if more support could be provided to help teams develop strategies: 
I think it is a great idea, but the management aspect of it is challenging. It is not like we can go some place and get this information; we have to compile it ourselves. Next year it will be easier if our target population still needs to work on context clues. It would have been helpful in the beginning, whoever thought of this idea, if they thought that a lot of schools would have vocabulary or comprehension as their skill, they could say, "Here are some materials you can use." That would have made our lives a lot easier.

Overall, teams' sources for developing instructional change strategies included teacher professional knowledge (e.g., adaptations of existing lesson plans or units), advice from coaches, internal consultants, or external experts, trade magazines or books, commercial curricula, or research journals. The majority of teams in the research sample adopted change strategies that were developed by team members or collaborating teachers. Less than a third of the observed change strategies were full adoptions or adaptations of externally developed instructional or curriculum materials.

Complexity of Strategies. Three general approaches were taken by Inquiry Teams to developing strategies to try to improve the performance of target students in the subskill areas. The simplest and most straightforward was to identify a single, rather specific strategy to implement for a period of time, with a pre- and post-assessment tailored to the activity. Examples of this approach included:

- a timed reading strategy with students to improve fluency;

- a commercially available program to help students learn multiplication facts;

- a specific strategy using "semantic gradients" for teaching context clues;

- a three-lesson sequence of activities for teaching a different set of vocabulary words each week;

- a "vocabulary detective" activity in which students identified words of interest in their independent reading books and developed strategies to determine the words' meanings.

The advantage of this approach was that measuring progress was relatively straightforward. All students received the same pretest, the same change strategy, and the same post-test. Hence, teams were able to draw some conclusions about whether the specific strategy employed had made a difference and for whom.

A second approach was to identify multiple strategies that would be implemented simultaneously to help improve student skills. For example, an elementary school that targeted reading comprehension (with a focus on identifying the main idea and supporting details) purchased reading kits that focused on reading comprehension to be used during the extended day program, developed a specific reading comprehension exercise for teachers to implement in the classroom, and ordered leveled books for classroom libraries so that students could choose more appropriate reading material. When multiple-strategy approaches such as this were used, teams tended to monitor progress through whatever assessment tools they customarily used, often a combination 
of state and district tests (such as the ELA exam and Acuity) and school-based formative tests (such as DRA or running records).

A third approach to developing a change strategy was to assemble a menu of strategies targeted at a specific skill and provide those to the teachers who would be working with the target students. For instance, a middle school targeted fractions, and the team analyzed data carefully and determined that there was a need for teachers to use more manipulatives, higher-order questioning techniques, and teach math vocabulary explicitly. The math coach, a member of the Inquiry Team, worked with teachers to help them incorporate these activities into their classrooms. At another middle school where the Inquiry Team focused on the use of context clues for the $7^{\text {th }}$ grade target students, the literacy coach and academic intervention teacher conducted professional development for classroom teachers to show them the specific weaknesses of the target students and then offered them a menu of instructional strategies to remediate these weaknesses, including read-alouds, phonetic-awareness activities, shared reading, guided reading, leveled books, and metacognitive activities.

A less common approach was to design a special program for the target population that was implemented before or after school, or through some pullout program. An elementary school that wanted to improve students' enjoyment of reading offered a kind of reading club after school to the target students and any other students at that grade level who wished to participate. A high school that targeted $11^{\text {th }}$ grade Global Studies developed an after-school program to help prepare the target students for the Regents Exam, with one teacher focusing on content and a second teacher focusing on test-taking strategies. The disadvantage of these kinds of programs was that students typically were not required to attend. In the case of pullout programs, it was sometimes difficult to find time to remove students from class to work with them. As a result, there were often several target students who did not receive the change strategy.

A variation on this approach was to target students who were in a single teacher's classroom and implement an instructional program with that class all year. This occurred in one high school that targeted ELL students who would be taking the Math A Regents Exam. The majority of the target students were placed in one teacher's classroom. The team selected a commercially available mathematics program to implement with these students. Unfortunately, due to scheduling issues, there were a few students who had to be placed in other teachers' classrooms and thus, did not receive the same change strategy.

Duration. Change strategies also varied in terms of the amount of time required for implementation. As discussed earlier in the report, because 2007-08 was the first year of the Inquiry Team process for most of the study schools, the first few months were spent getting oriented to the process and identifying a schoolwide focus and a target population of students. Many teams did not begin implementing a change strategy until January, and one as late as mid-May. 
The most common approach in terms of duration was to identify a strategy or series of strategies that would be implemented for the remainder of the school year, either in the regular classroom or in some before- or after-school or pullout program. A small number of teams implemented a short-term change strategy, monitored results, and then adopted a second change strategy. One school, for instance, implemented a commercially available program on multiplication tables to the target students and found that they made remarkable progress. The team then moved to an approach in which the math coach would provide mathematics strategies monthly to all $3^{\text {rd }}-5^{\text {th }}$ grade teachers - which encompassed the target students.

Cost. The cost of the change strategy included purchasing the change strategy itself or any resource materials required for its implementation. Costs appeared to be a limited concern for the teams at this stage of the process. Only a few teams purchased new curricular materials; the majority used programs, materials, and resources that were already available at the school or within the school district.

There were other costs involved, of course, including paying Inquiry Team members to serve on the teams. Some principals emphasized the importance of continuing to fund the Inquiry Teams because of the amount of time it takes to serve as a team member. One principal remarked:

There is not enough time and that seems to be an issue. I am not sure how to resolve it, but time does seem to be an issue. I also heard they were thinking of making these [Inquiry Teams] a full-time, but how can you have a full-time Inquiry Team during the day? So therefore you are pulling these people from classrooms; what happens to children? You have to provide the funds for it too, and still maintain low class sizes ... . I really would like to be able to supplement the funding if possible in order to expand the team and give them more hours or just get a bigger team. We spoke about a retreat at the last meeting, to take them away at the end of year and have a retreat and look at the implications for instruction of the following year. I would like to do that.

Teachers Implementing the Change Strategy. The target populations of students were sometimes concentrated in one or two classrooms and in other instances dispersed across the school. However, because the target groups were typically about 15 students, the number of teachers involved tended to be small. The majority of Inquiry Teams chose to minimize the number of teachers who would be working with the target students; the typical pattern was for students to be served by a maximum of 4 teachers. More often than not, one or more of these teachers were members of the Inquiry Team and thus, privy to first-hand information about the team's goals and priorities.

When teachers of the target population were not on the Inquiry Team, the challenge to team members was to find time and methods for sharing their work with classroom teachers, soliciting their participation, and ensuring that the teachers implemented the change strategy. One team dealt with this issue by asking the three implementing teachers 
to attend Inquiry Team meetings on a regular basis - even though they were not official members; this kept them in the loop and helped the teachers buy-in to the inquiry process.

An atypical case was observed in a middle school in which the 17 target students were spread across 14 classrooms. Team members reported that this was done to ensure that the change strategy would be implemented across the school; in effect, the entire math department was asked to implement the change strategies identified by the team.

While it was ideal to have the implementing teachers serving on the Inquiry Team to ensure that they were fully informed and supportive, scheduling of team meetings was a barrier to this in some places. With teachers on the team, it was difficult to schedule meetings during the school day, and some teachers were involved in after-school activities or graduate coursework as well. The challenge for Inquiry Teams was how to ensure teacher buy-in and implementation of the change strategy. The most effective approach seems to have been to have implementing teachers serve as full or ancillary Inquiry Team members.

Embeddedness of the Change Strategy. The change strategies could be woven into an existing classroom or program, or take the form of add-on programs delivered before or after school. The most common approach, which was adopted by about two-thirds of the study schools, was to embed the change strategy in the regular instructional program of the target students. This was done by asking the students' classroom teachers to implement specified strategies in the classroom. This approach not only ensured that the change was incorporated into students' regular learning activities, but had the additional advantage of sometimes affecting the learning of other students in the classroom. Rather than isolate the target population of students, some teams purposefully developed learning activities that teachers could do with all students.

At a high school, for instance, the team developed a vocabulary acquisition exercise that teachers of the target students could implement with all of their students. Two elementary school Inquiry Teams identified a specific reading comprehension strategy that they asked teachers to implement with all students in their classrooms, even though not all of the students were target students.

A much smaller number of teams (about one-fourth) chose to offer some sort of special program for the target population. This was accomplished in one of two ways. One approach was to develop a special before- or after-school program for the target population. An elementary school, for instance, offered an "early bird academy" that the target students were required to attend three days per week to focus on decoding and phonemic awareness. Other schools offered similar opportunities as part of their Academic Intervention Services (AIS) programs. This approach seemed most effective if students could be required to attend. In schools where student participation was optional, there were often attendance problems. In one high school, mandated student community service competed with the after-school program, resulting in less than $20 \%$ of the target population participating. In another school, despite assertive measures, student participation never exceeded $50 \%$. 
A second approach to offering special programming for the target population was for Inquiry Team members themselves to work directly with students in a kind of tutoring situation, which occurred in at least five schools. At a middle school, for instance, each team member was assigned 3-4 students. They worked with each student at least once a week during team members' prep periods on strategies the team had identified. At an elementary school, team members worked with their assigned target students in small groups at least three times a week, pulling from a list of strategies developed by the team.

The disadvantage of this approach was that it was sometimes difficult for team members to schedule time to work with students, and there were difficulties with students transferring their learning to their regular classrooms. In our conversations, one member of an Inquiry Team that used this approach wondered if the team had misunderstood how they should implement the change strategy.

I think that was maybe a misunderstanding on our part-that if we would try it out on this sample population and it would make an impact on student performance, then we would share that information in the class, because the students would be so successful everyone would want to know what we did. But we learned, number one, that the kind of work we were doing was not having the direct impact we thought it would for more than one reason. One is sometimes the students look at the activity you are doing as isolated and they don't understand how this will help them as readers. It becomes something I do with Ms. when she picks me up. In some ways it was good, because those students became a little more introspective about their own reading and what they needed to do to become better readers just by having someone coach them. But as I found out when I went to monitor one of my students, she was reading to her teacher and slipped right back to the same thing, and I am like, "What are you doing, that is not scooping?" But it did not occur to her that what she did with me is what you do all the time. So that is one of the reasons we thought that this was not making the impact we need. Working with the students individually is always good, but to have a bigger impact, we need to do something else.

Summary. As these brief descriptions suggest, Inquiry Teams were often resourceful in leveraging the knowledge of team members or colleagues to design or select an appropriate change strategy for their target population. Some teams struggled, however, to develop a clearly articulated change strategy and even those that did identify one found the process to be time-consuming and challenging. One surprise was that the network staffs were not typically used as a resource for supporting this search and for helping teams review the research base of alternative change strategies. In some cases, research team members observed Inquiry Teams at different schools working independently of one another to develop strategies to address identical subskills. This suggests that the Department and/or networks could provide more assistance in compiling effective strategies that address the skill areas targeted by several schools. 


\section{Evaluating the Effectiveness of Change Strategies}

Just as teams gathered and analyzed data to develop a change strategy, they were expected to continue gathering data as the strategy was implemented to determine its effectiveness. The majority of Inquiry Teams in the study sample conceived of this step as ongoing and formative in nature; a much smaller number (about one-fifth) viewed the evaluation as a final step, had not gotten far enough along in the Inquiry Team process to do an evaluation, or had let the evaluation step fall to the wayside. At the time the research team visited schools, only three of the study schools had completed one round of the change strategy, done an evaluation, and had moved to a second cycle. Over half of teams reported that they were engaged in ongoing monitoring of their change strategies. The remaining teams were not engaged in evaluating results for one of the reasons noted above.

Inquiry Teams typically used a combination of data sources to evaluate the effectiveness of the change strategy. The most common tools used were the state assessment, the Acuity or Performance Series periodic assessments adopted by the New York City Public Schools, and school-adopted assessment programs such as the Developmental Reading Assessment (DRA) and running records. Information from these more formal assessments was often supplemented with student work, classroom portfolios, observations, and interviews with students.

Teams that had developed a highly specific change strategy often developed their own assessment tools because nothing could be found that matched the strategy. For instance, a team that focused on science and social-studies vocabulary for ELL students identified high-frequency vocabulary words and crafted their own assessment tool to assess student understanding of those words.

Two high schools intended to use only one form of assessment to measure student progress - the state Regents exam. This was because their area of focus was to improve the preparation of target students to take a specific Regents exam.

Teams generally focused on monitoring the impact of the change strategy on the target population rather than monitoring teacher implementation of the change strategy. However, some teams did monitor implementation by conducting regular classroom observations or by asking teachers to report to the Inquiry Team on a regular basis. At one school, team members reported that after visiting classrooms to gather data on students, the team had expanded their change strategy beyond students to include teachers through a professional development series that the team was planning:

Where we are seeing a breakdown across the board is in instructional delivery that is affecting students, both inside and outside the target population... I think a specific type of professional development that is outcome-oriented could help. 
Because teams had started late, and began slowly to implement the inquiry process, few teams had gotten far enough along to make major adjustments to their change strategies based on what they learned from monitoring student progress. In the future, when Inquiry Teams have a full year to implement the process, they will more likely have time to do interim evaluations and adjust change strategies as needed during the school year.

In the Department's end-of-year school survey, Inquiry Teams across the city reported the progress of target population students on their June 2008 learning goals. In terms of this study, 38 of 41 participating schools reported results for 604 students. Across the 38 study schools, $71 \%$ of all target population students reportedly met the June learning goals set by Inquiry Teams. When also including students who "almost met" the June goal, the overall percent rises to $87 \%$. Teams ranged widely in their success, from a low of $42 \%$ of target population students meeting or almost meeting their June goals, to a high of $100 \%$. A table with the progress of each team's target population in meeting their June goals is available in Appendix F.

\section{Sharing the Work of the Inquiry Team}

Another important aspect of the inquiry process is keeping the broader school community informed about the work of the team, sharing ongoing analysis about student needs and findings regarding effective practices. Team strategies for reaching out to the school community hold significant implications for the acceptance of Inquiry Teams as legitimate and significant resources for school improvement.

Inquiry Teams in the study sample were evenly split on whether they believed teachers throughout the school were familiar with their work. The great majority of teams had made some attempt to inform teachers about their work at some point during the year, but the frequency and nature of communication with faculty and staff varied widely. About half of the teams in the sample engaged in ongoing communication, but often this dialogue was with a limited group of teachers.

The typical pattern was for teams to inform the entire faculty about their work early in the year (or in a few cases, at the end of the year), and to communicate regularly throughout the year primarily with teachers who served the target population students - or with teachers at the same grade level or in the subject area that was targeted. At a high school that targeted math, for instance, the assistant principal for mathematics was a member of the Inquiry Team and kept the mathematics department abreast of the team's work. Similarly, an elementary school team targeting $4^{\text {th }}$ grade students kept $4^{\text {th }}$ grade teachers informed of the team's activities during grade-level meetings. Teachers of the target students often were more intimately involved through occasional attendance at Inquiry Team meetings or through contact with instructional coaches who served on the team and supported the teachers as they implemented the change strategies.

The majority of teams (about two-thirds) used several forms of communication to reach teachers who were not directly involved with the team's work. The most common method 
was to share at faculty meetings, and/or at meetings by grade level, department, or instructional teams. One-third of the Inquiry Teams also used informal conversations or asked team members to turn-key the information in whatever manner they chose. Other forms of communication included newsletters or email communications, distributing minutes of Inquiry Team meetings to all faculty, and inviting all faculty to Inquiry Team meetings. Two members of one Inquiry Team created The Inquiry Team Inquirer, a newsletter to keep faculty members abreast of the team's work. At another school, the facilitator of the Inquiry Team contributed a column to the weekly e-newsletter distributed to grade-level teams.

A common feeling among members of the Inquiry Teams was that during this first year of implementation, they were not in a position to engage in any extended conversation because they were still learning about the process well into early spring. Members reported wanting to engage faculty, but were hesitant to do so until their teams had interesting and meaningful findings to share. Team members at about one-fifth of the study schools stated specifically that outreach to the faculty was a weak area for their teams that they hoped to strengthen in the future. One team member explained:

To be quite honest, we have two classroom teachers who are on the team, and they have buddied up with two other classroom teachers to share their work, so we have six teachers who do it. But it is not really schoolwide yet because we need more time to hone this. We need more time.

At the time of our visits to schools in late spring, a number of teams were planning or had just held professional-development meetings or informational sessions with faculty to share their work and solicit feedback about their findings. These presentations included their analysis of student learning needs, the selected change strategy, and some preliminary results. One school, for instance, held a professional-development event featuring an elaborate PowerPoint presentation and a video of the target students charting their progress for the year. Another team held an "Inquiry Team Day," advertised in advance with suggestive flyers with such text as "IT is coming" and "Are you ready for IT?" On Inquiry Team Day, the team hosted lunch meetings featuring a PowerPoint presentation describing the process and sharing the team's focus and findings for the year.

Teams that held such sessions reported receiving positive feedback from teachers, who were not only interested in the analysis but were ready with suggestions for next year's focus problem. A number of teachers expressed interest in joining. On the flip side of the coin were a very small number of schools (fewer than five) where Inquiry Team members believed their work was not supported by other teachers. One of these schools was in its second year of implementation and the team was requiring all teachers to engage in the inquiry process, which was resented by some teachers. Another school in its first year of implementation was quite large and was in a state of transition with a new principal and a factionalized faculty divided among teachers resisting new initiatives and relatively new teachers who were embracing change. An Inquiry Team member remarked: 
There is a perception that there is a certain group of teachers who assume leadership roles and are constantly selected for these things; they tend to be disproportionately young and from TFA [Teach for America], so that creates some tension. But our principal is fairly new, so one of the issues is-as with anyone coming into a new place-she has to find people to whom she can delegate responsibility, and some people may feel they should be in that group and are not.

In summary, the great majority of teams had made some attempt to inform the rest of the faculty and staff about their work. This often took the form of an introductory informational session at the start of the year. Typically after this initial introduction, only teachers affected by the team's work were kept regularly informed. Some schools, however, provided regular updates through newsletters or faculty meetings. Other teams were hesitant to reach out to faculty to share their work until near the end of the school year when they felt more comfortable with their own learning. There was hopefulness that more sharing about the Inquiry Team process would take place in 2008-09.

\section{Stimulating Larger Improvements in the School}

It is anticipated that as teams delve deeply into the learning needs of the target population and adopt change strategies to support student learning, they will identify improvements that could benefit the entire school. These improvements might be instructional or systemic. For instance, if a particular instructional strategy to improve vocabulary acquisition for ELL students proves effective for the target population, the Inquiry Team will likely want to use the strategy with ELL students across the school. Systemic improvements are intended to improve teaching and learning conditions across the school.

A little more than half of the Inquiry Teams in the study schools had developed plans to share the change strategy with the broader school faculty and had implemented those plans by the close of the school year. The biggest barrier to making schoolwide changes, then, was that this was the first year of implementing the Inquiry Team process and many teams needed more time to design and implement their change strategy. Another explanation is that some teams seemed singularly focused on their change strategy and did not examine the larger conditions of learning. Of those that did, they typically encountered these issues unintentionally while diagnosing their target populations' learning needs; in these cases, insight into needed changes in the conditions of learning often emerged as unexpected "aha" moments, rather than from systematic exploration.

Given the newness of the process, it is encouraging that just over half of the Inquiry Teams studied did attempt to disseminate change strategies beyond their target populations, with an additional $25 \%$ of teams planning to disseminate change strategies the following school year. The changes being pursued in the study schools were evenly split between instructional and systemic changes. 
Instructional changes were typically specific strategies or a set of "best practice" strategies that the teams had identified and used to help the target students improve in specific skill areas. After (and sometimes before) finding these to be successful, teams disseminated the strategies to a wider group of teachers. In one school, for example, a commercially available program for teaching automaticity of multiplication facts was purchased for the target population, but subsequently offered to all $4^{\text {th }}$ grade teachers. Similarly, another Inquiry Team determined that the target students could improve the targeted math skills if their teachers made increased use of manipulatives and higherorder questioning techniques, and placed more emphasis on mathematics vocabulary. The math coach then helped all math teachers implement these strategies.

The most common type of systemic change teams pursued focused on encouraging and assisting a larger group of teachers with the use of data to inform their instruction or using all or part of the Inquiry Team process with their own students. In several cases, when Inquiry Teams discovered the instructional power of the Acuity and/or Performance Series, they began offering assistance to other teachers across the school in the use of these systems. One school that was in its second year of implementing the Inquiry Team process had decided to take the entire process schoolwide. Each teacher was asked to identify a set of target students and a subskill on which to focus. The Inquiry Team provided the teachers with binders of data on their target students, and held weekly meetings with the faculty to provide support for implementing the process. In another school, the search for earlier student performance results revealed a disorganized filing system that had discouraged teachers from investigating students' past achievement. Student portfolios also needed updating so that they could become useful resources not only for the Inquiry Team, but for teachers and students alike. The Inquiry Team took action to develop a new policy and to physically organize the materials to support their use. In two other cases, teams discovered while visiting classrooms and talking with teachers that classroom libraries were deficient in leveled-reading books and dictionaries and thesauruses. These resources were then supplied to all classrooms. Another team noticed the assessment being used by reading teachers did not provide the kind of detailed information they needed for the target population. After locating a more effective assessment tool for the target population students, the Inquiry Team planned to have the entire school use the new instrument next year. Yet another team that had completed one change strategy discovered that the state assessment results showed more positive student results than student classroom performance. This Inquiry Team was planning an end-of-year professional-development day for teachers in which they all would dig more deeply into the data to identify actual student weaknesses and help the Inquiry Team determine a new focus for the upcoming school year.

Facilitators and Barriers to Schoolwide Change. As noted earlier, the fact that this was the first year of the inquiry process for most schools was a barrier to implementing changes schoolwide. It took time for the teams to establish themselves and gain confidence that their actions and insights were worthy of consideration by others. Similarly, team members at several schools noted that the time-consuming nature of the Inquiry Team process itself was a hindrance to taking the work schoolwide. Team members were overwhelmed by how much time it took to work through the process, 
including analyzing data and developing change strategies. Not only did the time they spent on the process delay any sharing with their peers, but they were concerned that it would be difficult for most classroom teachers to implement similar processes because of time constraints, particularly as the end of the year approached.

Other factors shaping teams' capacity to implement this stage of the process were connected to the school context. At the top of the list was the professional culture of the school. Schools that already had culture of data-informed instruction, change, and professional sharing found it easier to persuade other teachers to adopt successful strategies. Often these schools had already developed structures that facilitated sharing, such as common planning time and "lunch and learn" meetings, for example. On the flip side, some teams anticipated or had experienced difficulty taking their work schoolwide because the professional culture did not support change and sharing. One Inquiry Team member commented:

Some teachers' lack of buy-in, that would be the biggest obstacle ... getting everyone on the same page has been a bit of a struggle. They see the [inquiry process] as one more thing to do.

School size also was viewed as a significant factor in taking the work schoolwide; small size facilitated communication and sharing, while large size had the opposite effect. An Inquiry Team member at a large elementary school remarked:

Our school is extremely large and there are lots of teams and committees. At other schools there might be this known thing that the Inquiry Team is prominent and important, but I don't think at this school they could name the people on the team. So we meet internally and we are starting a process on Monday about bringing people on board.

The composition and expertise of the Inquiry Teams was another factor affecting the degree of success in taking the work schoolwide. It seemed to help if the Inquiry Team included teachers of the target population. Because these teachers had actually implemented the new strategies and found them successful, they were viewed as credible sources by their peers. Similarly, teams with skilled Data Specialists and/or instructional coaches were able to use these team members to provide professional development and support to nonteam members on data analysis and new instructional strategies.

Last but certainly not least, the support of the principal for the Inquiry Team process was pivotal in determining whether other teachers were willing to agree to make the changes. Active participation and support by the principal sent a message about the importance of the process. In addition, principals who supported the work were more likely to look for opportunities to spread the team's successes to a larger number of teachers and students.

In summary, for the first year of citywide implementation of the Inquiry Team process, about half of study schools reached the point of implementing schoolwide changes. Even so, about half of the Inquiry Teams in this study were able to spread successful strategies 
or structures beyond the target population and their teachers. These strategies were about equally divided between instructional and systemic changes. Most Inquiry Team members believed that next year the teams will be better positioned to engage faculty in their work throughout the school year.

\section{Sustaining and Deepening the Work Within the School}

One indication of whether Inquiry Teams will be able to sustain their work is their perception of the value of the process. Only if members find the process worthwhile does it seem likely they will to continue to devote the considerable time and effort needed to effectively implement the Inquiry Team process. At the close of our interviews, we asked each participant if they felt the Inquiry Team process had been valuable for their school. The overwhelming majority of responses were positive, some enthusiastically so.

Team Members' Views on the Value of the Inquiry Team Process. Over three-fourths of Inquiry Team members in this study believed that on balance, the Inquiry Team process had been worthwhile. The most common explanation they gave was that their teams had realized the value of the Inquiry Team process itself - that is, doing a detailed analysis of available information on struggling students and determining how to help them succeed. Team members reported that they valued the opportunity to get to know the students deeply through their detailed analysis of data. They also believed that they had learned how to use data effectively to help focus instruction. The fact that they spent so much time analyzing what was working or not working for a small group of students had led them to think about ways to help the entire school.

Team members at several schools reported that seeing tangible evidence of their students' growth via assessments and classroom observations was proof of the value of the Inquiry Team process. Several teachers who served on Inquiry Teams reported that their own teaching had improved as a result of what they had learned about data analysis and instructional strategies. Another benefit of the Inquiry Team process reported by several team members was that it promoted collaboration and unity of vision.

Only a handful of Inquiry Teams questioned the value of the process. Typically they were concerned about the amount of time the process consumed or they were from schools where the process had not been well developed. However, even in schools where teams valued the process, there was some hesitation to endorse it fully simply because of the challenges teams had faced in the first year of implementation and because a few teams were cautiously waiting for confirmation of their work in June. Even so, the vast majority of Inquiry Team members felt that in spite of the difficulties they faced, the process had been valuable and worthwhile for their school and for them professionally.

Principals' Views on the Value of the Inquiry Team Process. The majority of principals also spoke favorably about the value of the Inquiry Team process, although somewhat less so than other team members. About two-thirds of principals stated without ambivalence that the process was worthwhile. Some mentioned that the process caused 
staff to think more about personal and professional practices and about the needs of students differently, and had helped teachers take more ownership of student learning. Others stated that the process enabled their members to look more closely at data. One principal even asserted that of all the recent initiatives, this one was the most valuable because it allowed for flexibility and choice. These remarks from principals illustrate the positive responses:

It really helped us to focus on our strengths and weaknesses, and on strategies that we had been using, and more of individualizing and looking at data in a different way. And it helped us just because the school is so big, so we really learned how to do it in a small focused group, and now we are able to see it in the larger picture. I really like it; it has been amazing.

It was a learning experience but a good one. I was skeptical at first but sometimes you need something to focus you and this did!

Those who hesitated to fully endorse the Inquiry Team experience, like some teachers, were concerned about the time demands or the fact that there was some overlap with the work of other teams or committees. Some principals were overwhelmed by adding something else to their already heavy workload, and were concerned about devoting so much time and energy to only a few students. Said one principal:

Yes, the process is worthwhile but like everything, as we continue I would like to modify the process in a way that it would not affect programming so much. I wish we could just teach kids and focus on the classroom and not go through this whole big separate thing-I just want it to happen in the classroom.

Some principals expressed hope that the process will realize its full potential as teams become more experienced:

I think as we become more efficient in the process, it will be worthwhile. It is a change of culture, so it will take time. My particular educational culture, we are a little behind when it comes to the practice of open door and sharing of practice and inter-visitations here in the school, so building professional structures and sharing will take a little more time, but eventually if done properly, the practice will be very beneficial in improving instruction and having people professionally study their craft.

How to Make the Process More Valuable. When asked how the Inquiry Team process could be made more valuable for their schools, team members responded by suggesting ways in which the Department could improve the process and also how the school itself could make the process more valuable. The most common responses (heard from about one-fourth of the study teams) directed at the Department were that teams needed more professional development or guidelines about using assessment results and crafting 
change strategies. A nearly equal number remarked that the process needed to begin earlier in the school year, and that teams needed more time to do the work. A Data Specialist who wore many other hats at the school commented:

Give the people on the Inquiry Team less responsibility so they can devote more time to it; make it a .4 or .2 position so you don't have other responsibilities. The city really needs to look at that, especially for the Data Specialists, because I am pulling the data for all the teachers and then this work, too. It is great information that we can give to the entire school, but it is finding the time.

Other common responses were that there should be more interaction with other Inquiry Teams, that more funding should be provided for change strategies (heard especially at non-Title I schools), that all Inquiry Team members should attend the same professional development, and that the databases needed to be perfected and made more user-friendly. Some principals noted that because the ELA test was administered in January, it was difficult for teams to implement a change strategy long enough to make a difference for the students before they were tested.

Regarding how the teams themselves could make the process more valuable, the two most common responses were that more teachers should be involved on teams and that the team should do more sharing schoolwide or expand the work to include more grade levels and/or subjects. Some of the teams with no members who were classroom teachers noted that their presence on the team would make the process more valuable. Often these teams did not have classroom teachers as members because of the difficulty of scheduling meetings at times that teachers could attend. A few teams commented that parents of the target students needed to be more involved in the work.

Plans for the Inquiry Team Process for Next Year. In a majority of the schools, Inquiry Team members already were thinking about the possibilities associated with continuing the Inquiry Team process the next school year. Few Inquiry Teams had developed any concrete plans by the time of our site visits, but different ideas and scenarios were being discussed informally among team members.

A number of principals and team members were interested in expanding the work of their Inquiry Teams in some manner. A common idea was to sponsor multiple Inquiry Teams next year. For some, the desire to create new teams was driven by the possibility of investigating other problem areas for the school. Others thought new teams would help involve more teachers in the process, particularly if the Inquiry Teams could be integrated into existing structures such as department or grade-level teams. At least two teams were considering branching off and having each Inquiry Team member facilitate a smaller Inquiry Team so that more teachers and students could be involved.

A handful of teams had thoughts about identifying a different target population. The general feeling was that it might be more helpful to focus on students who were at slightly higher performance levels than the bottom third. The thinking here was that 
focusing on a different group of students might have a more positive result on the schools' test scores.

About $20 \%$ of the teams planned to continue working with and/or monitoring the target population with whom the team had worked in 2007-08. Some of these teams hoped to simultaneously target a second population of students with learning needs. As they thought about expanding the process by forming new Inquiry Teams, current team members noted the need to develop the capacity of others in the school to serve on Inquiry Teams. Many acknowledged the need to tap their experiences and thought a mentoring approach would enable new teams to find their own way more easily.

There also was interest in using the Inquiry Team process to establish content focused teams, which some thought would ensure that team composition spoke directly to the tasks of the team, particularly bringing together the expertise needed to diagnose student needs and develop and implement change strategies. At the elementary school level, there was some interest in focusing on the lower grades to ensure members could track students over time as they progressed through the school. A few teams were making plans to involve parents more fully in the process.

Several teams mentioned that they were waiting for further direction to see if the process would continue or change in any way before making recommendations for next year. Even so, as noted above, most teams felt positively enough about the first year's experience to begin conversations about how to make the process even better in 2008-09.

\section{Summary}

The information shared above reveals that the vast majority of schools in this study made substantial progress implementing the Inquiry Team process in 2007-08. Given the steep learning curve required to implement the process as intended, the level of understanding and implementation observed when the research team visited schools in May and June of 2008 was rather remarkable. Had we visited the schools earlier in the year, the story undoubtedly would have been different, as teams reported the frustration they had experienced in the early months in trying to obtain needed training and figure out what they were supposed to do. By the time we visited, however, the great majority of team members understood the purpose and steps of the Inquiry Team process in ways that closely matched the Department's vision. The majority of schools had worked through the steps of the process with fidelity, and had seen the positive results of their work. A large majority of team members found the process to be of value, and had begun making plans for continuing or expanding the work in the upcoming year.

There is room for improvement in 2008-09 as some teams felt frustrated and overwhelmed as a result of the workload, and some were adamant that for the process to succeed the Department would need to continue funding the work and provide even more support for implementation. The Department may need to give particular attention to helping schools develop change strategies, and to assisting them in using what they learn to effect schoolwide changes - an underdeveloped step of the process in 2007-08. 
Formative Study of the NYC Inquiry Team Process, 2007-08 


\section{External Support for the Inquiry Team Process}

The external support structure that became available to schools in the 2007-08 school year has the potential to strengthen the implementation of the Inquiry Team process. Each of the 41 schools in this study was affiliated with one of seven school-support organizations. Each SSO had a unique mission and provided a portfolio of instructional, legal, and consultative services and products to member schools, typically including opportunities to network with other schools. Within SSOs, member schools were typically organized into networks; schools in this study belonged to 18 networks, which ranged in size from 15 to 26 schools. Each Network Leader often managed a small team of specialists in core content areas, special student populations, and/or data/assessment systems. Network Leaders' descriptions of their roles and responsibilities were broad, comprehensive in scope, and service-oriented, and characterized as "anything principals need" and unlimited availability "24-7-365." All schools also were assigned a Senior Achievement Facilitator from the Department's Office of Accountability, who was responsible for supporting school-based Inquiry Teams and for helping schools access and use all of the new accountability tools, such as the Quality Review, Progress Report, and ARIS. Typically, SAFs were assigned to work with a particular SSO and to support schools in one or two networks.

The Inquiry Team process initiative was announced after the Department's selection of SSOs to serve city schools and after schools had selected their SSO partner. Schools in this study reported selecting SSOs based on a variety of factors, with about half identifying two or more reasons for their choice. Principals seeking empowerment and autonomy chose to affiliate with the ESO. Those seeking support tended to be affiliated with the ICI-LSO and the Knowledge-LSO, and those seeking an organizational and philosophical alignment tended to affiliate with the CEIPEA, the Leadership-LSO, and the Community-LSO. All principals, except for those associated with the ESO, said their decision also was influenced by a prior relationship with, or familiarity with, the SSO leader and/or Network Leader. In a handful of cases, the principal, along with others, first identified a particular Network Leader and then sought an SSO affiliation willing to support that choice.

Early in the school year, the Chancellor set an expectation that SSO Network Leaders and SAFs would work together to support schools during this first year of empowerment under the new structure. Collaborative Action Teams (CATs) were formed in each SSO to facilitate communication, but the nuts-and-bolts of this collaboration were left to individual Network Leaders and SAFs. CFI leadership held monthly one-day professional development sessions for SSO leadership and Lead SAFs to discuss different stages of the Inquiry Team process. The timing of these meetings paralleled the rollout of the Inquiry Team process at the school level, such that Lead SAFs and SSO Instructional Leaders took their learnings from these meetings to other SAFs and to Network Leaders who, in turn, used them to make plans and pull together materials to support school-based Inquiry Teams. 
This study focused on the work of the Inquiry Team and did not aim to examine directly the school-SSO relationships or the diversity and quality of services schools received through their Network Leaders. Most principals appeared satisfied with their SSO selection and their Network Leaders, and we heard some compelling examples of how SSOs were valuable sources of advice, educational services, and technical assistance to principals on a variety of school-based matters involving literacy, mathematics, special services for ELL students, human resources, compliance reporting, and community issues.

In this section we examine the particular nature of SAF and SSO contributions to the implementation of the Inquiry Team process and efforts made to coordinate this work.

\section{The Contributions of Senior Achievement Facilitators}

A vast majority of team members considered their SAFs to be the primary source of support for the implementation of the Inquiry Team process in their schools. Across the 41 study schools, the SAFs were able to establish working relationships in all but one school. This one case was at the request of the principal who felt a Department representative might "tamper" with her empowerment. Team members and principals characterized SAF contributions as "instrumental," "the major source of support," "the person who wants to help," and "the face" of the Inquiry Team process.

In reflecting on their work over the year, principals and team members identified a wide range of interactions with their SAFs. These contributions can be grouped as information sharing, knowledge development, and process facilitation. They include the following:

- provide information about Department initiatives and/or expectations about the Inquiry Team process;

- $\quad$ provide support in understanding and using the accountability tools;

- offer professional knowledge and experience to teams during discussions;

- provide training in ARIS, Acuity, low-inference transcripts, or other topics related to data use (directly or through network staff);

- serve as a liaison with other schools, sharing other approaches to the Inquiry Team process;

- serve as a "sounding board" to help assess the potential effectiveness or fit of the team's latest work with the goals of the Inquiry Team process;

- stimulate team thinking through questioning strategies or the posing of alternative scenarios; 
- offer advice to troubleshoot issues; and

- affirm the team's work and provide encouragement.

The SAF typically was characterized as a team's "guide" throughout the process. As the above list of interactions suggests, the SAFs were highly valued as a source of technical and social support. The venues for these contributions included SAF visits to the school, but also external meetings or trainings, often sponsored by the network.

Information Sharing. Teams talked about SAF contributions in terms of the information and expertise they brought to the table. In this role, the SAFs served as the primary, if not sole source for information about the Inquiry Team process and its connection to the accountability tools, such as the Quality Review and Progress Report.

She's been an amazing support. She has a wealth of knowledge. When we have questions of what to do now or next, she's been able to put the Inquiry Team in perspective with the school Progress Report, and make those links, and show us how the work of the Inquiry Team could impact it. She attended at least six meetings this year. She's been very available. She's done a fabulous job - brought resources, supported us in our direction, and hasn't brought her own agenda, but supported ours.

The major contribution of the SAF has been the knowledge that she has that the Department has given her through their meetings.... She walks you through the steps you need to know to be confident you are going in the right direction and covering all bases. She will call and ask how things are going. She is very supportive, even on a personal basis. If she is [nearby], she might just stop in and talk to me or to the Data Specialist, so she is very supportive and has a lot of information to get out to us, so I am very satisfied with the SAF.

The SAFs were highly valued for providing teams with information and materials on the Inquiry Team process. As one team member exclaimed, "Thank goodness for the SAF or else we would have been fumbling." Their contributions included some of the materials supplied by the Department through the monthly design meetings, but also templates and graphic organizers that SAFs sometimes developed on their own.

She came a couple of times, was very helpful, gave us a graphic organizer, flow chart showing how they are expecting the team to work and how other schools have done it, gave us a direction and a structure. At first we were not quite sure. She got us going, came back again and we showed her our hypothesis. She gave us great feedback that we were doing well and let us know we're on track. 
Only a few teams, however, reported actually receiving guidance about the pacing of the Inquiry Team work or key benchmarks in the rollout of the process for the entire school year.

Many SAFs were a source of material and human resources for Inquiry Teams. They provided principals and members with professional literature on formative assessments, data analysis, and differentiated instruction, among other topics. These materials appear relevant to local team needs and interests. Explained one principal,

[The SAF] has been here a number of times and this is excellent. She seems very in tune with what we are doing. She knows us individually: where we were and where we are now. She gives us materials to read: books, articles. She gives me suggestions and a monthly newsletter to me and the Data Specialist.

Many SAFs also were viewed as bringing a professional knowledge base themselves, regarding data use, literacy, ELL strategies, or special education, for example or experience working with teachers or implementing similar programs. In addition, SAFs were recognized by members for offering ideas and suggestions based on their professional experience. A few commented that SAFs' past roles as local instructional superintendents and principals were important resources.

Knowledge Development. Second, teams talked about SAF contributions in terms of helping to build their knowledge base to engage and complete the Inquiry Team process. Some SAFs provided training for teams, typically around student data systems, such as ARIS or Acuity, or introducing new tools, such as low-inference transcripts, to support their work. In some cases, SAFs conducted the training; in others, they were viewed as a resource broker, bringing in data experts from the network or outside vendors. The following accounts from a teacher, principal, and coach, respectively, reflect these informational resources.

Our SAF is very involved. Personally, if we have questions, she guides us every step of this process. She sent an email giving us a checklist of where we should be. She gives letters about professional development. She gives professional readings which we will use soon. She is a source of information and offers very personalized meetings specific to our school. She is fabulous.

She was great. She came and checked in to let us know we were on the right track, what we could be doing. She gave us a sheet to see where the holes are in our work. She taught us how to do low-inference transcription, which is less threatening than a classroom observation. She gave us good information.

[The SAF] was very helpful. She came to a few meetings, offered more support, more professional development, anything we wanted. I would feel 
comfortable calling her up and asking her anything. She was very helpful with developing and implementing instructional practices and monitoring the target population. She gave us information on how to do these things. She provided direction, gave me a little professional development one time on a model for a classroom rubric to help monitor student progress. I shared that with a math teacher. She told us next steps, programs, or curricula that might be helpful to students.

Teams highly valued SAFs who served in a liaison role by sharing information about the work of other teams in their network. In a few cases, SAFs provided opportunities for teams to meet face-to-face to share strategies and challenges, an outreach that was highly valued by team members.

We have sat down in our other meetings with other schools to share what is going on in our schools, and taken little ideas from different places to incorporate into our school. It helps us realize whether we are falling behind, whether we are ahead. [It's] just an opportunity to share and learn.

We did go to another school not long ago and met with a couple of other Inquiry Teams in our network to find out what they are doing. [Our SAF] set that up, and that was really helpful. It was good to see their good ideas or things we would want to avoid.

Process Facilitation. A third set of SAF contributions to Inquiry Teams was their attention to facilitating and strengthening the team process itself. In this regard, a majority of teams valued SAFs who participated as active team members and were willing to begin the conversation wherever teams were in their thinking. Many team members were pleasantly surprised to find that their SAFs played roles similar to that of critical friends or informed consultants, rather than being directive or demanding, as some had feared. Again, a teacher, coach, and principal, respectively, share their views:

[Our SAF] gives us suggestions and it's up to us to decide.

She poses questions and asks you to do the thinking.

She questioned our work when it needed it.

Overall, most teams felt a strong ownership of the process, which many connected to how SAFs contributed to their team meetings directly.

Most teams felt interactions with their SAF improved their decisions and strategies. Teams reported using SAFs as "sounding boards" for ideas, new plans, or data analysis to gauge if the team was heading in the right direction. SAFs were valued for asking questions that stimulated the team's thinking, which tended to call members' attention to missing or overlooked aspects of the work, or potential consequences to team decisions 
that could prove problematic. One principal characterized this contribution as "persistent in a good way." This strategy seemed to have motivated some teams to think more deeply about the actual Inquiry Team process, its meaning, value, and role within their particular school. Questioning by SAFs also helped clarify for some teams their end-of-year goals and strategies for attaining them. One member explained,

When we met with her we had an idea what we wanted to do regarding our change strategy. [She] especially would prompt us with certain questions, what we were doing, why we were doing it. She got us to think about the end goal and where we were going and how to get there. She was pushing us to think more about what we were doing.

SAFs also were described as helping teams brainstorm about how to address a new problem or issue. As a "troubleshooter," SAFs were credited with helping teams "get unstuck" at particular stages of the process, for example, identifying a target population that would be representative of the school's student population.

She's been very helpful because there was a point in the process when we were stuck. In addition to ELLs in $4^{\text {th }}$ grade, we had other questions in the other grades. She focused us to look at the NYSESLAT.

When our principal felt we came to a frustration point, the SAF came in and stayed with us for a meeting. ... She was very instrumental with that. She looked at Acuity and explained it to our data person and gave him access.

In this role, teams described SAFs who took time to sit with the team, or by email, work through several options for resolving problems. Teams were usually pleased with the SAF assistance in troubleshooting, which permitted them to move on to the next stage of the process.

Finally, SAFs were valued for attending to the psychology of the Inquiry Team process by building team members' confidence in themselves and in their work. As a single group working on a new process in their school, an Inquiry Team typically had no other sources of support aside from occasional team sharings. Explained one principal: "The key is that we were not left to do this alone. We were supported through the whole process so that made it easier for us to bond as a team, and [it] relieved the anxiety." In several cases, SAFs were valued for being "positive" and "upbeat" and for giving the team feedback that reinforced some of their work, specifically progress made to date that highlighted the strengths of their decisions. The following member comments point to this form of support:

[Our SAF] is a good resource and support.... She encourages you and gets excited when you excel, like when you share success stories.

She is quite resourceful, comes with encouragement and support. She's 
thrilled to hear what we have done so far.

Our SAF is a great cheerleader.... [She] believes in our work.

Given the time-consuming nature of the work and the uncertainty at times, SAFs played a valuable role in boosting team members' confidence by affirming their work and encouraging them to move to the next step.

Level of Support to Teams. There was some variation in the type and intensity of support from SAFs as reported by principals and team members. To examine the variation we developed a typology of support levels drawing upon member descriptions of their interactions with SAFs. These levels and their distribution across the 41 schools in this study are presented in Table 7.

Table 7. Variation in the Type and Intensity of SAF Support.

\begin{tabular}{|c|l|c|}
\hline Level & Definition & $\begin{array}{l}\text { Percentage } \\
(\mathrm{N}=41)\end{array}$ \\
\hline High & $\begin{array}{l}\text { Attended team meetings 4+ times over full year, } \\
\text { training \& material support, advice, and network-level } \\
\text { hosting }\end{array}$ & $42 \%(17)$ \\
Moderate & $\begin{array}{l}\text { Attended team meetings 2-3 times, training and/or } \\
\text { material support, advice, email support }\end{array}$ & 32\% (13) \\
Low & $\begin{array}{l}\text { Some principal, Data Specialist, or chair contact } \\
\text { through network meetings, attended one team meeting } \\
\text { and/or email support }\end{array}$ & $24 \%(10)$ \\
None & No recognition of SAF name/role & $2 \%(1)$ \\
\hline
\end{tabular}

Overall, almost $75 \%$ of teams reported a high to moderate level of support from SAFs including multiple on-site visits to team meetings, some training and material support, and regular exchanges through email or network meetings. About a quarter of team members reported limited interactions with SAFs or that SAF interactions focused on the principal or the Data Specialist, typically at network meetings. Schools with high SAF contact were associated with all SSOs except the ESO. SAF levels of support in schools were moderate or low. As mentioned earlier, the one school reporting no SAF recognition was the one where the principal requested no SAF involvement. This typology was developed from school descriptions and therefore may not align with Department expectations regarding the work of SAFs with individual schools. 
Figure 3. Team Member Descriptions of Different Levels of SAF Support.

A school in which team members experienced a high level of support from their SAF:

[Our SAF] is extremely involved and very supportive. She attends our meetings, organizes the [network] meetings, and has organized a smaller group of schools within our network who are focusing on ELLs in vocabulary, so she has been great. Even when she attends Inquiry Team meetings, she lets us know if we are on the right track, do we need to go in a different direction....She really helps us kind of think in a different way, which is helpful.... I like the way she approaches the team because she doesn't come in as an authority figure. She listens to what we have to say, and if we are not heading in the right direction, she does not say it is bad, but she does explain the benefits or negatives of what we are doing. If anything, she has been a source of information. She provided us with templates, information —one on high-level questioning. It is quite useful.

A school in which team members experienced a moderate level of support from their SAF:

A lot of her work was around working with the data. And she has come to two of our meetings and met with the data specialist and the principal. She is a kind of mentor to help us with this process, give us feedback, and shar [e] directional changes. From the training she receives, she in turn brings it down to us. She brought in the data specialist to deal with data intensively, going through ARIS and all the new websites that we are still learning to navigate. That was important to get us started in the data process and gathering the data; she was a facilitator there. Our SAF meets with us and tells us the process, the expectations. She pulls from all schools she has worked with. She comes to our meetings and observes and brings in suggestions. She brings in books that we should do for book studies. She came and observed what we were doing. She just sat and listened to how we were moving, and she said we seemed to be going in the right direction.... [Also] she has been good because she tells us where we are going off track and steering us back, so that has been helpful. She has given us the sense of being comfortable with our learning. So I would say she has been fairly active. She observed, provided training, [was] very friendly, nonthreatening. I think she did a very good job.

A school in which team members experienced a low level of support from their SAF:

I only met her once. She has always emailed if we need help and has visited .... They could come and intelligently ask questions about the work. Being more prepared to speak and offer support [would be helpful]. If she is not part of the team, how can she be a part of the discussion? I'm not sure what the role is. ...She hasn't come to many meetings - [she]only came to one meeting that I know of. We're all on different schedules, and there were no guidelines. What did [the Department] expect from her? Was it just to put Acuity in place? How involved do you want her to be in the Inquiry Team beyond finding out what we've done? I think the principal is very capable, and if she thought we weren't in a position to meet our goals and aims, then she would have asked for help [from the SAF]. But our team is capable. Right now we feel we get enough support. 
Given this variation, how satisfied were principals and teams with SAF support? In reflecting on their SAFs' work, principals and members tended to be relatively in sync in their views. There was a strong correlation between satisfaction and the level of support schools received from their SAFs. Figure 3 offers composite descriptions drawn from multiple school-based interviews to illustrate these different levels of support from SAFs and team satisfaction.

Those schools that had received high to moderate levels of support —about $75 \%$-were positive and expressed satisfaction with the support provided. Those that had received a low level of support - about 25\%-emphasized that while the team, principal, or Data Specialists had benefited from some contact with the SAFs, most wanted more support, and shared specific ideas on what kind of support was needed. In fact, the kinds of support these members requested paralleled those offered by SAFs in high-support schools. This suggests that SAF's work was strongly valued, even to those who had not yet taken advantage of this role or who realized they would like additional support.

Three schools offered a somewhat negative opinion of SAF contributions, each with an unusual context: one principal who rejected support for philosophical reasons; a midyear principal change that led to some confusion over the initiative and SAF/network roles; and a high-capacity school with a large Inquiry Team that was confident and independent-minded. Of note, principal opinions about the quality of SAF contributions did not appear connected to their role in the Inquiry Team as chairs, contributing members, or nonmembers.

\section{The Contributions of SSO Networks}

Another source of support for the work of Inquiry Teams was network membership with the newly established SSOs. A majority of team members felt their particular network had little or no impact on their work. In a fifth of study schools, the network was unknown to team members or had not provided support. These schools were concentrated in three SSOs. In contrast, almost one third of study schools reported receiving two or more forms of support for their Inquiry Team work, typically principal or "specialist" meetings sponsored by the network, training on assessments or data use, and/or attendance at 1-2 team meetings at the school. This level of support was similar to low or moderate levels of support provided by SAFs. Four SSOs provided a mix of such services to study schools. Some of these supports, however, were concentrated in one team member, such as the Data Specialist attending network liaison meetings. As noted earlier, the Data Specialists often played an important role in leading and facilitating their Inquiry Teams. Half of schools reported receiving only one form of support from the network, typically focused on the principal, who may or may not have been a team member. For these members, the network was a rare or infrequent presence in their work. Table 8 provides a summary of team descriptions of how network staff contributed to their work. 
Principals and Data Specialists tended to have more contact with network staff than did other Inquiry Team members, and they tended to be more positive about network contributions in general. In contrast, many team members expressed neutral or no views about network support, simply because they felt they did not know enough to have an opinion. The SSO structure and the Department accountability mechanisms appear to place a strong emphasis on strengthening principal relationships with Network Leaders. In terms of the Inquiry Team process, this emphasis may have narrowed school-level awareness of network capacities and resources, with more focus on the principal's role.

Table 8. Variation in Interactions Between Inquiry Teams and Network Staff.

\section{Primary Exchange}

Network staff unknown to Inquiry Team members or not viewed as providing support for Inquiry Team process

Interacted with principal and/or Data Specialist at network meetings, one-on-one meetings, or by email

Trainings in assessment or data use (e.g., ARIS and Acuity)

Attended Inquiry Team meeting (1-3 times)

Provided professional development on a topic requested by the Inquiry Team

Sponsored event for teams to share their work

Mix of the above
Percent of Schools $(\mathbf{n}=\mathbf{4 1})$

$20 \%(8)$

$20 \%(8)$

$15 \%(6)$

$10 \%(4)$

$2 \%(1)$

$2 \%(1)$

$32 \%(13)$

Networks were mostly useful to teams in providing technical trainings around ARIS or assessments and for hosting meetings to facilitate learning and some sharing among principals and Data Specialists. It is not clear how much network meeting time was devoted to the Inquiry Team work. The range appears to run from 15-minute updates to three-hour sessions on "best practices," usually delivered by SAFs. Visits to team meetings by network staff were typically concentrated at the beginning of the year and focused on establishing teams and providing advice regarding the schoolwide focus and selection of the target population.

As already noted, in most schools team members identified SAFs as the primary sources of support. Yet there were a handful of cases where Network Leaders were providing the same level of support as SAFs; however, the schools did not associate the team process with the SSO. In these cases, the contributions appeared somewhat balanced, with both SAFs and network staff providing low or moderate levels of support to teams; SAFs did tend to meet teams in person, while network follow-up was by email. These examples of balanced support across these two roles tended to be concentrated in schools affiliated with the ESO. Only two teams associated the Inquiry Team process with their Network Leader and not their assigned SAF, and these were in ESO affiliated schools; in both cases, the level of support provided by the Network Leader was described as low. 
Many principals characterized their network as an emergency or fall-back source of support. For these principals, network resources appeared latent, typically characterized as "support as needed" or "support when needed." Some principals seemed proud not to have tapped network support much during the year. The ESO principals tended to espouse this philosophy, but some principals in other SSOs shared similar views.

\section{The Network Contexts for Supporting Inquiry Teams}

As mentioned earlier in this report, SAFs and Network Leaders had great discretion in determining how to support school-based Inquiry Teams. It is not surprising then that different work patterns emerged across the seven SSOs and the 18 networks in which study schools were organized. These varied in the degree of coordination between SAFs and network staff in terms of information sharing and leveraging existing network resources for the Inquiry Team process. This variation largely reflects the discretion that Network Leaders exercised in shaping the SAFs' work environments.

Across almost all networks, one clear and common approach was to identify SAFs as the primary resource for schools regarding the Inquiry Team process. This clear division of labor reinforced two overarching patterns of support reported by study schools: (1) SAFs were team members' main sources of support for the Inquiry Team process, and (2) network support was present in about $70 \%$ of schools, but typically at a low level and isolated to principal conversations and/or training around data use. Exceptions were found in schools working with the ESO because some Network Leaders wanted to continue supporting their year two schools directly.

Within this adopted division of labor, Network Leaders varied in their stance towards the SSO role in the Inquiry Team process. Almost all were supportive of the concept but viewed it as a "department initiative" and therefore outside the purview of the SSO and their network. Most Network Leaders were guarded towards the involvement of SAFs in their work, at least initially, because SAFs reported to the Office of Accountability. Network Leaders felt this might threaten their discretion and, more importantly, their relationship with principals as confidential sources of support. Some Network Leaders maintained cautious relationships with SAFs throughout the year. This concern was particularly strong in situations where the SAF and Network Leader had no preexisting professional relationship to build from. Even so, by midyear most SAFs and Network Leaders forged some agreement about how to work together, and most established a positive or neutral working relationship that did not detract from the other's commitments. There were a few cases of continued passive indifference at the presence of SAFs or role conflicts around data support; in these cases, an uneasy coexistence was established and SAFs worked independently.

Network Leaders held positive attitudes towards the Inquiry Team process. Many had sat in on an Inquiry Team meeting in one of their schools, some because the principal had asked, some because they were curious to see the process at work. Few Network Leaders 
were able to talk in detail about the Inquiry Team process under way in study schools, and their information typically came from conversations with the principal or comments made during a network meeting. In a few cases, the SSO's data expert had more interactions with teams than did Network Leaders.

Network Leader views conveyed some misperceptions of the implementation of the Inquiry Team process, which may reflect their limited contact with teams as well as some deeper assumptions about the tensions between support and accountability. For example, Network Leaders felt that most schools were implementing the Inquiry Team process more out of compliance than voluntarily. While this may have been true in the beginning of the initiative, there was little sign of this mentality by the close of the school year when CPRE visited schools.

Second, most Network Leaders held a black-and-white view of the SAFs' affiliation with the Office of Accountability as one of "enforcement," "directives," and "evaluation." They expected SAFs would develop negative relationships with schools and that this might, in turn, undermine network standing with principals. This expectation, however, was not born out in study schools. To the contrary, most team members described SAFs as collaborative and facilitating their ownership of the Inquiry Team process. Even in schools where SAFs made strong suggestions that teams revisit the broad nature of their schoolwide focus and target population, most admitted that this rethinking was needed. In fact "support" was a common word used to describe most SAFs with high to moderate levels of interactions with schools. These misperceptions on the part of Network Leaders may help explain the arm's length distance at which some Network Leaders held SAFs throughout the year.

A majority of SAFs shared a similar philosophy and many of the same strategies towards working with schools, although there appeared some variation in the material supports used to inform Inquiry Teams about the process. And while their work environment was more or less positive, and some were allowed to leverage network meetings or data experts for their work with Inquiry Teams, SAFs did not appear dependent on network support to do their jobs. Where network meetings did not exist or leaders did not invite them to attend, SAFs either set up their own meetings or visited schools more frequently. This was true in three SSOs, where the level of SAF support to study schools was typically high.

Beyond their network affiliation, SAFs felt their work was impacted by juggling additional, but sometimes related, job responsibilities as Lead SAFs or Community or High School Superintendents. Those in the superintendent roles felt particularly stretched because their schedules often were subject to unexpected political issues or principal hiring decisions that needed their approval. In addition, SAF/superintendents were responsible for an additional 30 or so schools. While these obligations were different from the intense work of the Inquiry Team process, there were cases in which small problems in one school could take a few days to resolve. Other responsibilities, such as leading Quality Reviews in other schools, also stole time from their Inquiry Team schools. In this study, we found no association between these extended responsibilities 
and levels of support provided to Inquiry Teams. Tracking the impact of these different SAF workloads may require an alternative research design.

Degrees of Coordination Between SAFs and Network Leaders. Within this context, Network Leaders and SAFs developed different approaches to coordinating the work of the Inquiry Team within the network. These approaches appear motivated by a number of desired outcomes - to preserve the independence of staff, either SAFs or Network Leaders; to provide schools the support needed; and to minimize instances of competing services or conflicting advice. The different approaches to coordinating support reflected a continuum of strategies that ranged from coexistence to collaboration.

One approach to coordination was a form of parallel play in which the SAF and Network Leader pursued their work on independent tracks. In these cases, the division of labor was fairly strong. As the relationship evolved, SAFs and Network Leaders exchanged information about schools in trouble through short email "alerts" so either party could address issues as he or she deemed fit. Also, if the network hosted regular principal or specialist meetings, the Network Leader would include an occasional focus on the Inquiry Team process, but instead of seeking the SAF's advice, the leader would call principals or team chairs directly for "best practices." In these situations, some Network Leaders avoided working with SAFs directly, which created an environment of uneasy coexistence in which SAFs and network staff pursued their work independently.

In a few other cases, Network Leaders talked about making sure they "reinforced" or "backed up" the Inquiry Team process in their schools through emails or conversations with their principals. These verbal reinforcements were often informal and unplanned. In this role, Network Leaders occasionally asked principals how the work was going and, if asked, sat in on an Inquiry Team meeting. Sometimes this led to offering advice about team composition or about the process itself. Network Leaders then made a decision about whether to share information with their SAF. Explained one Network Leader:

Some schools call us in, and we happened to be there on that day-no rhyme or reason unless a school called and asked me to be there due to a problem. So, if they call us to come and [there's] something they want us to focus on, I go. Some have, some have not. It is always a question we bring up though.

As this example suggests, Network Leader attendance to meetings was often opportunistic and driven by requests from the principal.

Another form of coordination was in the sharing of network meeting time. In this case, Network Leaders asked SAFs to present information about the Inquiry Team process during the network principal or specialist meetings, e.g., for assistant principals, coaches, or Data Specialists. SAFs used this opportunities in various ways, sometimes to provide short updates on the current focus of teams' work and how the process was going. In other cases, it was used to highlight best practices, which might involve presentations from teams about their work. 
Another form of coordination focused on redistributing work responsibilities across both SAFs and network staff. This included responsibility for monitoring the Inquiry Team process in network schools and/or running specific network-sponsored meetings. The ESO was an unusual SSO because it had piloted a similar initiative to the Inquiry Team process the previous year. To honor this experience and prior relationship, a couple of SAFs agreed to work only with new schools, leaving the support of the year two schools to network staff. A common belief across ESO Network Leaders was that year two schools needed little support; this approach was reflected in the low (or no) levels of support provided to a majority of year 2 Inquiry Teams in the study. In four networks that held regular principal and specialist meetings, Network Leaders asked SAFs to take responsibility for running the network Data Specialist meetings because of their expertise in data use and/or overlap with the Inquiry Team work. In these cases, the Network Leader provided updates at SAF Data Specialist meetings, and SAFs provided updates at Network Leader principal meetings. A few SAFs and Network Leaders reported attending each other's meetings "to be supportive" and to learn about any new developments in the schools.

For a handful of SAFs and Network Leaders, initial efforts to coordinate work evolved from that of preventing missteps to a professional collaboration in which SAFs became viewed as valuable members of network teams. In these cases, SAFs and Network Leaders routinely shared information about new developments at the schools and brainstormed about what supports were needed and next steps. Sometimes they also visited schools together as a team, but then split into their respective roles, with the Network Leader talking to the principal and the SAF to team members. On rare occasions, both visited the Inquiry Team together. Often these covisits led to a collaborative discussion around school needs, sharing information and strategies about next steps. In a few cases, either principals or other Inquiry Team members noticed the quality of the coordination between Network Leaders and SAFs. One principal explained,

The Network Leader works along with the SAF, and they are not in isolation; they have developed a relationship and support each other. The SAF has the opportunity to come to all our network meetings, so there is continuous support for the Inquiry Team or whatever we need to focus on as a network.

In two cases, Network Leaders and SAFs saw complementary areas of strength in one another and used those strengths to develop a division of labor to support network schools with attention to the Inquiry Team work. In these unusual partnerships, the division of labor was split into data use and instruction. In these cases, the SAF and Network Leader did not often visit schools together, but rather coordinated schedules based on school needs and the expertise each could provide. Each maintained an independent schedule focused on their different roles, but responded to requests from the other based on evolving school needs. This arrangement was limited to the Network Leader and did not extend to other specialists on the SSO staff. 
There were cases where the work of SAFs was embraced and more formally situated in the work of the SSO. In one case, this took place at the organizational level of the SSO, such that all SAFs were included in SSO-level planning meetings and strategic discussions about individual schools. One SSO official explained:

We embraced the SAFs and the Inquiry Team as good additions to school improvement. We treat them as members of our leadership team and early on embraced the work of the Inquiry Team. We bring a different expertise and focus but so do they. We share documents with them. We invite them to our cross-collaboration meetings in which we debrief what is happening in each school — and we talk about the Inquiry Team work-next steps, whether we need more support, or to redirect or help the principal, seek levels of professional development, or the SAF works her angle.

In this case, the Inquiry Team process was viewed as a strong school-improvement initiative that was similar to prior work supported by the SSO. In addition to information sharing to strengthen both their efforts, this SSO created a specialist meeting for Inquiry Team members that met regularly. All of the schools in this study associated with this SSO received high levels of support and completed a full cycle of the Inquiry Team process.

Within individual networks, a few leaders also chose to embrace their SAFs as additions to their staff and to see the Inquiry Team work as important to support. This approach was viewed as a way to manage potential conflicting roles between the SAF and network staff charged with supporting data use in schools. It also was viewed as a way to extend the expertise and reach of the network. Strategies for embedding the work of SAFs in the network included joint visits at the start of the school year to emphasize their unified approach and to pair network staff, typically data coaches, with SAFs to provide support in schools throughout the year. This effort to embed SAFs in their work at the start of the school year often reflected Network Leaders' personal view of the importance of the Inquiry Team process as a school-improvement strategy. In some cases, this strategy was facilitated by prior working relationships between the SAF and the Network Leader. One result was that from the school perspective, the SAF sometimes was viewed as a network resource, not a Department representative. When two principals were asked to describe the network role in the Inquiry Team process, they stated: "They gave us the SAF" and "They got us together with the SAF."

As this discussion suggests, in extreme cases efforts to coordinate the work reflected a reluctant coexistence between SAFs and network staff. But in others it created a division of labor that leveraged network communication channels and the professional strengths of SAFs and network staff. For the most part, SAFs were able to work within these different arrangements and in some cases to gain access to network meetings that amplified their visibility and support to schools. 
What we see missing from these coordination efforts, however, was a willingness to examine how network instructional resources might align with the potential needs of the Inquiry Teams over the course of the school year as their work evolved. Teams needed guidance in identifying change strategies. In only a few cases did this take place. In one example, unusual in its intensity, a Network Leader provided a literacy workshop for the Inquiry Team, reviewed scores of the target population with them, and offered advice for fine-tuning the change strategy in use - all at the suggestion of the SAF and principal. This was a powerful application of network resources and expertise, and one that took place due to the relationship that developed between the SAF and Network Leader. More typically, however, attempts by SAFs to encourage network instructional resources for the Inquiry Team process encountered resistance, mostly passive. Even in situations where Network Leadership and SAFs had positive, even collaborative, relationships, suggestions about using network instructional expertise for teams were reportedly dismissed by Network Leaders and staffs who felt "uncomfortable" in that role.

Relationship Between Work Context and Intensity of Support. As this discussion suggests, there are some patterns worth noting across these 18 networks and 7 SSOs regarding network contexts and the level of support provided by Network Leaders and SAFs. The SAFs were viewed as the primary source of support for school Inquiry Teams. Most Network Leaders provided indirect support through informational conversations with principals and short sessions at network meetings. This approach was reflected in team member perceptions of low or no support from their networks.

Networks also varied in the degree of coordination between SAFs and network staff ranging from a coordinated coexistence to an authentic collaboration. In the former case, the Network Leader simply reinforced the importance of the Inquiry Team process through check-ins with principals. This coordination was largely controlled by Network Leaders. The SAFs took advantage of the opportunities available in their networks, and supplemented those not available with other strategies. Whatever the situation, most SAFs were able to provide support to Inquiry Teams - to "make it work" as one SAF described. This may help explain why no clear pattern emerged in terms of level of support from SAFs and the different types of coordination allowed by Network Leaders. Another explanation is that patterns were weak because of the newness of the work for all and the evolving nature of the SAF-Network Leader relationship over the course of the school year. Still other explanations center on the study's research design, which included a limited number of schools from each network.

As noted, some schools in this study reported receiving a moderate level of support from both the SAF and network staff. These cases reflected situations in which support for the Inquiry Team was coordinated and drew upon expertise of both the SAF and network staff as needed. For example, both the SAF and Network Leader attended meetings at different times and shared different expertise, one on data use, the other on instruction. Other cases involved the use of the network data coaches to provide on- and off-site training for team members. In these cases, the level of support was balanced between both parties. 


\section{Accomplishments of the Inquiry Team Process, 2007-08}

What were Inquiry Teams able to accomplish during the first year of citywide implementation? In what ways might we expect the work of the Inquiry Teams to have positive impacts on their schools? In this section, we identify areas in which the Inquiry Team process can make a positive impact on teaching and learning. The Inquiry Team process aims to improve schools from within and to provide schools with a process that harnesses data-informed inquiry to stimulate changes in both instruction and school-level systems that result, ultimately, in improvements in student learning.

The following questions probe the accomplishments of the Inquiry Team process in its first year of implementation and beyond:

- Were school-based teams able to complete a full cycle of the Inquiry Team process?

- Was the Inquiry Team process able to develop team capacity to engage in a reflective study of the team's school?

- Was the Inquiry Team process able to foster a commitment to data-informed inquiry among team members and/or within the larger school community?

- Were Inquiry Teams successful in influencing teacher practice? and,

- Were teams able to stimulate larger improvements in their schools?

This study can begin to address these questions. However, to answer most of these questions fully will require data that are longitudinal in nature to understand how the initiative evolves over time and with what effect. Future research also should aim to understand the views of other teachers and administrators not serving on teams, or even student experiences with the Inquiry Team process. Even so, data from this formative study can provide insights into the accomplishments of the Inquiry Team process during its first year of citywide implementation. In particular, study site visits took place near the end of the school year, when CPRE's conversations with educators likely reflected their experiences with the full process and well-developed views on the meaning and value it held for them and for their schools.

\section{Completing a Full Cycle of the Inquiry Team Process}

A major challenge to the effectiveness of any school improvement initiative is whether it is implemented fully. Partial or superficial implementation is a common problem that undermines many innovations adopted by public schools. It occurs when only some, but not all, of a reform's components are put into action. Sometimes this is due to a design flaw; the initiative is not well developed, lacks coherence, or is difficult to understand. 
Sometimes it is due to misplaced assumptions undergirding a program or initiative about the will and local capacity - e.g., resources and expertise-needed for implementation. Generally in such cases, the innovation has to be tweaked or overhauled to better suit the adopting communities, or additional professional development and support are needed.

Given these common occurrences, some important initial questions for the Inquiry Team process in its first year of citywide implementation are these: To what extent were schools able to engage and implement the main components of the process? Did Inquiry Team members consider the initiative "doable" in their schools? Did Inquiry Team members consider the process valuable to their schools and therefore worth their time?

Completing a Cycle. In these 41 study schools, all teams reported holding discussions and planning at each of the key stages of the Inquiry Team process. By the time of the CPRE site visit in late spring, 54\% of schools had actually implemented the Inquiry Team process through the final stage of stimulating changes in the larger school through recommended changes in teaching or the conditions of learning at the school. This percent rises to over $75 \%$ of schools, if we extend the implementation cycle into the early part of the 2008-09 school year, when about a quarter of teams planned to hold professional development for teachers based on their learnings from the 2007-08 work. These teams chose to wait until the start of the new school year to maximize teacher buyin or to allow time to review June results to confirm the effectiveness of their change strategies. A short case study of a school that was able to fully engage the Inquiry Team process is presented in Figure 4.

Table 9. Variation in Implementation Status Across Study Schools During the Final CPRE Site Visit.

\begin{tabular}{|l|c|}
\hline \multicolumn{1}{|c|}{$\begin{array}{c}\text { Implementation Status } \\
\text { (May-June 2008) }\end{array}$} & $\begin{array}{c}\text { Percent of Study Schools } \\
(\mathrm{n}=41)\end{array}$ \\
\hline Implemented key stages of the Inquiry Team process & $54 \%(21)$ \\
Planning professional development to stimulate school- \\
level change
\end{tabular}

As noted in Table 9, one school was just beginning to select a change strategy at the time of the CPRE site visit in May. Among the factors contributing to this delay were the work overload of the Data Specialist, infrequent team meetings, a new principal who was overwhelmed with other responsibilities, the newness of the work, and a professional culture that did not support peer visitation. The team's unusual composition also may have contributed to the delay; the team did not include assistant principals or classroom teachers. Despite this limited progress, members were optimistic about the following school year. One member noted:

It is basically a work in progress. It was new to us, we had to become familiar with it. Finding the time to get together on a regular basis to stay 
focused on that was not easy. Then finding the right tool to diagnose the children and take it from there and make sure that we got into classrooms... And I believe that no matter what missteps we had, next year because we know more about what we are doing, it will make a difference....

The other 19 teams reported feeling behind schedule and identified some of the obstacles they were encountering. All talked about the challenge of finding common time and enough time to meet. In one school, the Inquiry Team was temporarily suspended while its members served on the Quality Review team. Some of these schools had professional cultures with limited or no history of collaboration; a few had deep divisions. Other reasons cited were a slow start-up or pace due to teams' revisiting early decisions regarding the schoolwide focus and target population or troubleshooting problems with evaluating their change strategy because the target population was spread across many classrooms and the teachers were resistant to classroom observation. As a result, these teams had less time for implementing and monitoring change strategies and even less for considering the implications of their findings.

The Feasibility of the Process. Conversations with team members about the value they saw in the Inquiry Team process and what recommendations they would make for improving the process provided another view into high levels of engagement in the 200708 school year. Most members felt the process was doable given the right amount of time and support. In addition, team members' suggestions for improving the process were largely focused on tweaking the implementation at the school level and receiving more detailed guidance about team pace and effective practices. The design of the Inquiry Team process itself was not framed as a problem by the teams.

This optimism also may have been enhanced by the strong fit between member capacity and interest in the work, given that most volunteered or were invited by the principal to join and be part of a team. It may also have been affected by the pride and sense of accomplishment the teams felt about their work at the end of the school year. This was most visible in the large data binders they created to hold their work and in the positive relationships they had developed with target population students.

Factors Influencing the Pace of Implementation. A number of factors facilitated team capacity and interest in completing the Inquiry Team cycle in the 2007-08 school year. Team members noted the support received from their SAFs, who were credited with helping teams move forward by informing, stimulating, and affirming their work, and of equal importance, helping them maintain ownership over the work. The personal responsibility that some team members felt towards the target population was a powerful force for developing a change strategy and making sure similar children in the building benefited from their work. The principal's role was an important factor: A principal's participation as a contributing team member and resource finder signaled that the team was engaged in important work. 
The research team also observed that Inquiry Team capacity to complete the work was supported by system-level inputs. On the technical side, these included materials and human resources dedicated to helping the teams complete the work, specifically laptops, extensive databases, the external SAF position, and the school-based Data Specialist. The fact that members were paid per session and were provided with laptops sent strong messages that this was an important initiative, supported by significant resources and clear Department support. Finally, the Department surveys of team work in December and June and the end-of-school sharing sessions sponsored by some networks created a community for this work. These communities, we believe, provided critical opportunities for feedback and confirmation. According to team members, these sessions worked to affirm the validity of the Inquiry Team process, helped team members see the possibilities of the work through the approaches of their peers, and brought an important dimension of face-to-face accountability regarding team decisions and progress.

Other aspects of the work may inhibit future team productivity and member commitment to the process. Most team members were surprised by the workload and, in turn, the demands on their time. The intensity of these demands may have been aggravated by the small size of some teams and by the composition of some of the teams. It also may have been associated with the delayed start, which shortened the work period and, in turn, intensified the work. These conditions proved particularly difficult for teams that needed to take additional time to access needed expertise, or to recruit the teachers responsible for the target population and who needed to be brought up to speed and convinced of the merits of the proposed changes.

Looking towards the upcoming year, most of the study schools were not planning for just a repeat of their work in 2007-08; they were focused on streamlining implementation within their schools and expanding the reach of the inquiry process to other teachers and students. The level of commitment to a second year of the initiative among current team members was relatively high. Said one teacher, "[The Inquiry Team] is something that is necessary and becoming a solid program. So I would want to be involved with this." The positive response from most members across this sample of 41 schools suggested strong momentum for the continuation of the Inquiry Team initiative at the school level, and this momentum should work to strengthen implementation in 2008-09. 
Figure 4. Full Implementation of the Inquiry Team Process by a First-Year School

\section{The Inquiry Team Process at City Elementary School, 2007-08}

City Elementary implemented the Inquiry Team process for the first time during the 2007-08 school year. By May, 2008, the team had completed the implementation cycle for the first group of target students, saw growth in their learning, and had implemented some of the more successful changes schoolwide. The following case provides details on what the team considered a successful first year.

In June, 2007, the principal learned that the Inquiry Team process would be implemented across the city and took advantage of summer professional development for principals on new data systems to support the work of the new team. By start of school, the principal felt she had a good understanding of the process "and where we needed to go as a school." The SAF assigned to the school made several visits in early fall to help the school form the team and identify the focus and target students by the November deadline. An Inquiry Team was formed made up of the principal, the two assistant principals, the math and literacy coaches, the IEP teacher who served as data specialist, and a third-grade teacher.

Based on state assessment data, the Inquiry Team targeted reading comprehension, with a specific focus on the subskills of reading comprehension. Within reading comprehension, the team looked at identifying main idea and supporting details, and distinguishing between relevant and irrelevant information. The team focused on $5^{\text {th }}$ grade students because they had better data on these students than those at other grade levels. They exempted special education and ELL students on the rationale that these students already received many additional instructional supports. The team selected 15 students from three of six $5^{\text {th }}$ grade classrooms to limit the number of teachers with whom the team worked this first year. They were careful to select teachers they thought would work well with the team. The three $5^{\text {th }}$ grade teachers attended many of the Inquiry Team meetings to discuss and report on the instructional change strategies they were using with these students.

With input and involvement of the three classroom teachers, the Inquiry Team developed reflective reading exercises for teachers to implement in their classrooms with the target students. These exercises were based on a method used with the Teachers College reading program. As they implemented these exercises, the teachers found that the DRA reading assessment the school used did not provide detailed or accurate information on the students' reading comprehension abilities, which led to a decision to use the Teachers College Running Records for these 15 students.

The Inquiry Team also determined, based on reading logs teachers had students maintain, that the students were not selecting books wisely, and often abandoned books before finishing them. This led the team to order more high interest books for classroom libraries; the teachers also talked with students about their reading levels and about how to choose books. (continued on next page) 


\section{The Inquiry Team Process at City Elementary School, 2007-08 (continued)}

All 15 students participated in the before-school AIS program, however, members felt the current reading curriculum used in the program was too general. To address students' learning needs, the team researched and purchased reading kits designed to provide more specific instruction on reading comprehension during this time period. The literacy and math coaches assisted during the before-school program to provide more adult assistance with the target students - and to give the coaches, who were members of the Inquiry Team, more direct information about how students were responding to the new approach.

Throughout the year, the team reviewed available data on the target students' reading comprehension, including state assessment data, the DRA, Teachers College Running Records, the Acuity predictive assessment, and classroom records and anecdotal reports from the three teachers. Data from the second Acuity predictive assessment were shared in spring with faculty, showing progress for all but four students.

The team has taken two of the practices implemented for the 15 targeted students to the school level: (1) helping all teachers level their libraries and ordering more high interest, low-level reading books; and, (2) implementing reading kits during the extended day program for all $3^{\text {rd }}-5^{\text {th }}$ grade students. Next year they plan to replace the DRA reading assessment with the TC Running Records schoolwide, which they did for the target population students this year.

All team members believe the process has been worthwhile, and all team members said they would serve on the team again. Two members noted how the inquiry process had helped the school identify flaws in its assessment practices and programs and contributed to the school's capacity to use data.

I think just asking us to zoom in on a targeted population has helped us see things for the greater good of the whole population, not just that group. And that was a big goal. We had been using the DRA for awhile and this was a new outcome for us-to see it [DRA] doesn't give us the clear picture we needed. The extended day program has been here a couple of years now, and we have talked about the need to give teachers something different from what they use in the room. This year we focused on what those tools might be. So that has been effective. [Team Member]

To me, the greatest benefit has been working with the teachers and children and finding specific individual needs that the children needed to move along. I almost felt there was an IEP for each child. You could really find out what the children needed, so maybe some of the grades won't reflect it, we can't be judged by one test. We see the growth in the classroom and the relationship between us and the children growing. It made us dig deeper and really reach the children's needs. [Principal] 


\section{Developing Team Capacity to Implement the Inquiry Team Process}

The Inquiry Team process is predicated, in part, on the capacity of a group of schoolbased educators to function as a team. During the first year of implementation, one observable accomplishment was how well team members worked together and were able to grow together as a team. Data from this study identify some of the capacities that team members felt were critical to their work. These capacities include attitudes and beliefs about the inquiry process, but also the skills that members associated with teamwork and data analysis. These capacities include

- clear understanding of the goals of the Inquiry Team and the purpose of its many steps;

- ability to work collaboratively and to seek wide participation among members and multiple perspectives on a given question or problem;

- attention to balancing the distribution of work across members in ways that take full advantage of team expertise and emerging opportunities;

- commitment to school improvement;

- knowledge of different assessments and how to interpret student results; and

- knowledge of effective instructional strategies in a given skill or subskill area and/or with particular student populations.

Interviews with three-quarters of the teams revealed evidence of these capacities at various levels and distributions across team members. These capacities were described in comments such as the following:

It was a learning experience. It showed us where our deficits are. And it showed us that if we can move the bottom third, we can move anyone...it was a positive experience.

It was a learning experience. I used to hate the word data. Now that I know how to use it to move students, it's a whole new world for me. I have a new respect for it after the Inquiry Team and the Quality Review.

It's been a pleasure to be part of this team. We're here for the same goal-help kids and help teachers. It has been challenging because of changes in the testing. But it makes me personally aware that running a school is for the kids' sake and success in life. It takes all of us to push the school ahead. I think every teacher should have the opportunity to do this! 
We worked together as team. It's a nice process and I feel part of a learning community. It's stimulating to hear my colleagues' ideas.

We all had our own parts. It's not like we all took on every aspect. We delegated....The Data Specialists always had the data available for us. The guidance counselor came up with the idea of adopting the students. Our administrator handled attendance and could inform us on students' attendance. Me and the other teacher could contribute how the students were doing.

It's just been a really good learning experience. A lot of work and a lot of time, but I learned a lot. [CPRE: What did you learn?] To see a different side of education, not [just] the teacher side, but the implementation side. How do you come up with what people use? I can see from both perspectives now. We're usually told as teachers what to do.

Yes, it helped me to take a closer look at the lower-functioning childrenyou know, numbers and things that are happening in your school. But it became personal too. I got to know the children and learn. And realize you were here to make the difference and know what is being done to help the child.

I think in a sense it will be more worthwhile the second year when we actually have a foundation so that things are implemented a lot more efficiently and quickly and we are able to move through the cycles. But it takes time. It is brand new.

As mentioned earlier in this report, of those team meetings we were able to observe, twothirds were high functioning, scoring between 21 and 25 . These meetings reflected a strong sense of the Inquiry Team's purpose and its connections to other aspects of the school such as instructional practices and school structure. The meetings were characterized by broad participation among all team members, however small or large the team, and the meeting structures reflected established routines and flowed smoothly and efficiently. These strong team capacities were shaped, in part, by careful selection and encouragement by the principal and by the strong personal interest of members in participating on the teams. Clarity about the purpose of the Inquiry Team evolved over the course of the school year through first-hand engagement with the process and interactions with the SAF and the "turn-keying" of information obtained from network meetings. Many teams wanted to increase their efficiency with the process, which was evident in the planning under way for 2008-09.

Knowledge about assessment literacy and instructional practice were spread across the team as members learned together about assessment choices and selected change strategies. Typically, one or two team members were in a position to share their expertise and guide others in its application. Understanding of the team change strategy was typically somewhat shallow beyond the implementing teachers, and most members were 
dependent on one or two others who were more knowledgeable about its design or selection and implementation. The exceptions were those strategies connected to Acuity. While team members talked about becoming more confident and even committed to using student performance data from multiple sources to assess learning, there was less conversation about increasing their understanding of the range of instructional strategies available to address students' diagnosed learning needs, e.g., students' inference making. These are impressions drawn from interview data and from team approaches to these stages of the Inquiry Team process discussed earlier in this report.

In looking forward to 2008-09, we would add one more capacity that team members will need to develop to be successful. Team members want to hear about other teams' work and to have input from other teams regarding their work. If networks provide such opportunities, teams need help becoming skilled in providing constructive feedback to other teams. One team member explained,

We need to learn from the experiences of others. It's like a football game you learn from looking at the tape. They're on the outside looking in and may see things that we can't see. They can tell us different [approaches].

For these exchanges to be productive, team members need skills in how to provide critical feedback and ask stimulating questions. Strong feedback would help other teams improve their inquiry process, enhance their reflections, and give them confidence to move forward. Some teams may need special coaching, particularly those in schools with limited histories of collaboration and those who feel superior about their achievement. Team capacity to engage in this kind of dialogue should be encouraged and supported.

It is important to note that the conditions of team members' work influence how well they can nurture and tap these important capacities. What it takes to complete the Inquiry Team work - the frequency and length of meetings and time for members to complete tasks on their own which fuels team conversations - is not clear to those who have not experienced the process. Most members were surprised by the time required for almost all work. The 2008-09 school year will offer an important contrast since most of the teams intend to start the process in September (if not August) and most members felt their experience would bring a higher level of efficiency to their work as well.

\section{Increasing Commitment to Data-Informed Inquiry}

At its core, the Inquiry Team process is an empirically-oriented approach to school improvement. Throughout the process, teams are expected to draw continuously on a variety of data - from student performance data to background information to classroom observations and measures of school environment - to diagnose and address student learning needs. They are expected to use data to evaluate the effectiveness of the change strategies used with target populations. During the first year of implementation, another accomplishment for the Inquiry Team process would be the degree to which school-based educators gained a new awareness of and commitment to data-informed inquiry. This 
commitment might be first visible among the Inquiry Team members. As the team shares its work with others, we would expect an increased interest in and commitment to data on the part of other faculty and administrators in the larger school.

The study did find evidence of strong commitment to data use, in general, and the Inquiry Team process, in particular, among a large number of Inquiry Team members. It is important to note, that the teams in this sample were weighted with individuals who expressed curiosity about student data, which suggests educators with this disposition and background may be the first responders to this new initiative. Recalls one teacher on the attraction of joining the team, "Everything is now data-driven and I wanted to figure out what that means." The second wave of responders may bring different interests and experiences. A small percentage reported feeling highly comfortable using existing datasets and the technology required to access them. Most Data Specialists felt well suited to the technical demands of their new roles as helping teams learn about, access, and use data throughout the process.

Teams expressed a strong interest in the new data sources available through ARIS, Acuity, and Performance Series. These new sources were attractive to educators not only for their flexibility, but for the immediacy of their feedback. They saw close connections between these sources of data and their instructional choices. One teacher explained,

I think without [the data], we would be lost. It has been really helpful to us. Without it, there is not a way to drive our instruction. We drove our instruction to this target based on the data given to us. We would not have known where to begin. So based on all this info, we were able to hone in on the skills [students] were lacking and target our population and drive instruction.

Even though a number of teams struggled to manage the many data sources, this did not undermine their commitment to use data.

The data is very good. It's broken down into categories that we have never seen before. My problem is classroom teachers are very busy people. Even though you introduce them to Acuity, it's about when do they get a chance to implement these tools in the classroom to advance their children?

Teachers have data on their own and the question is, What do you do with this data? What data takes precedence? Which is more important? How do you translate all of this into instructional learning?

The commitment to data-informed inquiry on the part of so many team members was clearly associated with the Inquiry Team process itself, so much so that it is difficult to separate the two. Valuing the process and data-informed decision-making were both associated with three dimensions of the work. First, the work was viewed as reconnecting teachers with uses of student data for instructional purposes. Some members reported feeling disconnected because of the recent emphasis on the uses of data for accountability purposes and on the development of large-scale data warehouses. In some cases, the 
inquiry process appeared to help team members revalue student data as a resource for their classroom practice. Two teachers explained this shift in thinking that occurred during the school year.

I always thought, and as a parent too, that all the testing is overwhelming. It is one more thing for teachers to squeeze in, it means more meetings and discussions about how will it impact the children. It is imposed on teachers and did not grow up internally. But I have to admit, as the year went on, I felt less of this and I started to think it might be worthwhile.

We didn't have this when I was a teacher. I like getting down and dirty with the data stuff. It excites me to know what is out there, where our kids stand, where we stand as a school. I like the notion that in the long run we can help these kids. At the end of this process, I know that we're looking at the future of what we should be doing. How do we alter programs? I want to get to see that what we created works.

Others reported an increase in their commitment to use data because of the emphasis on identifying effective change strategies by the Inquiry Team process. One teacher described the attraction of sharing "what works" with other teachers:

I really bought into the concept that what works for these [16] ELL students, these strategies that are working for them can be applied to another 200 students in our building that aren't getting these strategies. To be able to turn to teachers and say, Hey, this works, try this! is so powerful. You just turned 16 into 200, and that is why we are here, to move kids like this. I think it's a great idea.

For others, the process was not only a bridge connecting data and instruction, but a unique collaborative experience. A coach and teacher describe how the interactive dimensions of the work have increased their belief in the power of data to "force [educators] to look at things differently" in their school.

The [data] quality is wonderful. We look at ELA scores from last year, DRA levels from start of year. We have baseline tests, diagnostic tests, predictive tests. [CPRE: How well can you explore the data?] The team was very open and this openness makes a difference. ...the cooperative atmosphere and the discussions [within the team]. You can take risks. You can give comments that helps you to develop the intention of this Inquiry Team process. I think we all believe in this. This truly makes sense for in the end it serves kids much better. It forces you to look at things differently with criteria.

It's more than what I expected because we get the opportunity to have detailed, rich conversations about what can actually work and what is not working. Everyone bringing something to the table is valuable. 
Conversations are fulfilling. The work I did looking at data specifically was rewarding, helping move children along was important. It was an opportunity for interaction with colleagues. We all came together on different levels, sharing ideas and thoughts about children and their data.

In that regard, the process emphasized the use and interpretation of data $-a$ shift credited with capturing the interest and commitment of team members. One longtime school leader characterized the process as reflecting the "general sea change" at the Department in that "people not programs" were being emphasized as the means of strengthening teaching and learning.

Finally, the commitment to data-informed inquiry was seen in actions by team leadership that aimed to simulate a broader evidence-based culture in the school. The hope is that the teams will become change agents in their respective schools, promoting the value of data and modeling how data analysis can inform instruction and other school-level improvements. A third of teams in this study appeared to be taking steps in this direction, most planned, some implemented. This was a challenging step, framed as an "obstacle" by a few. Explained one teacher,

I think the team uses the data when possible. The obstacle has been to expand that to not only us to use it, but to get everyone to use it-so the [issue is] buy-in from staff.

Team commitment to increase awareness and use of data among school faculty was reflected in two strategies. One involved professional development on new assessments team members found useful - typically Acuity, but also protocols for looking at student work - to demonstrate their value and to provide faculty with the training needed to use them on their own. Other team members realized that the Inquiry Team outreach efforts during the year - the sharing of the process, findings, and interim products of their work - also presented opportunities to influence faculty views and build leadership capacity. Since many members did not join the team with aspirations of being school leaders, this awareness grew slowly over the school year. Explained one teacher in a school in which the Inquiry Team had regularly shared their work with faculty:

I feel like a leader. In the beginning of the year I did not think that way[the Inquiry Team work is] like taking on a different job almost and we have had to kind of set the example of looking at research and student work to improve practice. I think teachers see us in that role and they are getting that too. Next year [we] will look at assessment during professional development and take that on. Next year will be focused on student work and what that tells us...We're taking that hint from CFI.

Overall, team members who became committed to data use and saw value in the process also wanted to share the instructional value of data with faculty. For most teams this was associated with their desire to continue data inquiry at the school through the Inquiry Team process itself. Most schools wanted to expand the process either through creating 
more teams or by selecting new cohorts of target population students to diagnose. Most schools wanted to involve other faculty directly in the process by expanding team size or starting new teams.

\section{Influencing Teacher Practice}

The Inquiry Team process is intended to help each school close the achievement gap in student performance. Changes in instruction, specifically the differentiation of instruction to fit students' individual needs, are the primary vehicles for achieving this goal. In this regard, the inquiry process has the potential to influence classroom practices in the school by affecting the teachers who serve as members on the Inquiry Team, the teachers responsible for target population students who help design and/or implement change strategies, and the broader school faculty the team reaches through consultation and training.

Across this sample of 41 schools, to what extent had the Inquiry Team process influenced teacher practice? This formative study focused narrowly on the views and experiences of the Inquiry Team members and did not collect information from other teachers in the school. Although our data about classroom practice is self reported, it is significant that a large percentage of teachers who served on Inquiry Teams, about three quarters, felt participation on the Inquiry Team had influenced their practice, and most were willing to continue on the team and advocate for more teacher participation.

I had a great experience overall. I enjoyed doing it and I think it improved my skills as a teacher. I can take what I learned to the next classroom I teach. I understand how to use data to drive instruction. I know how to do that now. Overall the experience was great. We worked hard this year and I would love to do it again.

Teachers participating on Inquiry Teams also reported thinking differently about their practice, in terms of classroom management, instruction, or assessment, as a result of the experience. For example, many teachers reported changing their minds about an instructional strategy because they had gained deeper understanding of it through the analysis and planning of the Inquiry Team. This spanned all experience levels. Here a veteran teacher, a beginning teacher, and a first-year teacher share their new appreciation for small group learning and differentiated instruction.

I thought it was worthwhile for my kids and me [and] I assume for the other teachers as well. It has been a positive experience. I have been teaching 26 years, but [the Inquiry Team] has changed the way I think of my small groups because when I started it was textbook teaching. And although I did small groups, it was never as intense as it is now, with observations and conferencing, etc. One of my Inquiry Team kids made 103 points progress this year and I think it is because of the differentiating of instruction and really zeroing in on her. [Veteran Teacher] 
Yes [it has been valuable]. It put a whole new outlook on teaching. I have only been teaching three years, but in college it was about curriculum; you have to follow Bloom's Taxonomy, and [there was] nothing about what you do at the end [of the lesson], when half the class didn't get it. No one said they don't learn it just because you teach it. Data and assessment put that light bulb on, and that is a whole different way to look at information. That has helped. [Beginning Teacher]

[The process] makes every teacher a little more reflective. It forces you to really look at what you are doing in your classroom. You could just teach that [skill] and not do that assessment. Why do I teach the whole group of students and not see what these students are doing? I had heard of the concept of differentiation, but I had not really understood it...how it can be effective. [First Year Teacher]

Even when the target population was different in some respect from the students in their classroom - for example, focused on ELLs or special education students - teacher members saw implications for their practice. Here, general education teachers talk about the cross-fertilization between the Inquiry Team work and their classrooms.

I did not know what to expect in the beginning. ...It has been a lot more useful to me as a general education teacher than I thought it would be. [CPRE: How so?] Just what I found working with the target population students. I have been able to use with my own students.

It is very interesting work. I have a couple of ELLs in here, and it has changed the way I work with them. I now know the kind of text they need as opposed to my general education students. So I know at the start of the year I can give them more targeted materials. So I know what works for them now. [CPRE: You wouldn't have had that without the Inquiry Team?] No, because I would not have had the opportunity to study these kids and see what works for them and what doesn't. So in these 15 kids, I have a low, medium, and high in my class. I have kids on all the spectrums of learning, so now I know how to work with these ELLs at the various levels.

For other teachers, participation made them aware of the connections between their classroom practice and school level policies and routines and even the influences of the broader school system. Teachers are often isolated within their classrooms, and serving on Inquiry Teams provided a way for them to not only connect with others but also to see how their practice was connected to other aspects of their school. This awareness came through exchanges with team members who brought different perspectives and expertise to the inquiry tasks and led them to explore the many factors that can shape a learning situation. Two teachers shared some of the particular sources of influence for their practice: 
It has been professional development in itself for me. It makes me more aware of the educational system in the city. It has helped me look beyond the school to the city.

Having that bird's eye view of everything is important for teachers. With children in your classroom we can develop a narrow view...I think having the opportunity to look at children that we selected so closely and look at their background, different situations and be able to discuss what we can do to help makes me look at my own students differently. It helps me to look at my teaching differently. There are so many things that need to be addressed; we looked at health, parent communication, and things involved that make that child be in that situation academically. There are so many different factors when you have that expertise [of the team] looking at children in that way. I want to do the same for the students in my class. It's great to communicate with a team to build up the student. I feel lucky to be a part of this - having the time [to look closely at students], knowing this process will help me the rest of my career.

Only a few teams reported seeing changes in teacher practice more broadly. The lowinference transcripts were useful in this regard. One teacher thought the value of the process was its impact on those teachers who worked with the target population. She explained:

I think absolutely [the process is worthwhile] because already I see an impact. I was in the rooms yesterday; there is a huge difference between this observation and the last one. I could see the interventions that we suggested, I could pick them out. Not all. The teachers are just getting their feet wet with this process too.

During this first year of implementation, one important accomplishment was that teachers who participated directly in the process felt the work of the Inquiry Team had made important contributions to their views and practices as teachers. We know from conversations with participating teachers that many were attracted to the Inquiry Team because of the possibilities it offered for professional growth and the opportunity for reflection on their practice. And as this discussion has suggested, for three-quarters of the teachers in this study, the Inquiry Team experience met these expectations.

\section{Stimulating School Improvements}

Another significant outcome of the Inquiry Team process was the new knowledge generated about improving student learning within a particular school. Teams generated new knowledge by diagnosing and treating student learning problems and by conducting broader reviews of the decision-making systems in their schools that frame the conditions of learning. This outcome was dependent upon teams successfully implementing earlier stages of the Inquiry Team process. It also was influenced by the Inquiry Team's status 
within the school and acceptance as a legitimate leadership body by administrators and faculty.

An important indicator of accomplishment for the Inquiry Team process in the first year of citywide implementation would be evidence that teams had made recommendations for changes in instruction or other aspects of the school that impact student learning. An even more important indicator would be evidence that these recommendations had been implemented by the team through professional development or were being investigated further by administration or by teacher leaders to understand the extent of the identified problem and the value of the proposed solutions.

During the first year of implementation, teams reported mixed attention to this role of stimulating school improvements. Only about half of teams attempted to influence teacher practice or the wider systems that compose the school. In some cases, this was accomplished by raising questions with the educators who controlled or were responsible for implementing the policies, such as guidance counselors or librarians. In others, it was through well developed professional development workshops that prepared teachers to use specific strategies or assessment methods. Team insights were typically offered as "suggestions," particularly to other teachers. Some placed their recommendations in mailboxes; others passed them out at voluntary meetings. Many were not sure if teachers were using their recommendations. At this stage of the Inquiry Team process, it was difficult for teams to reach out to and engage other teachers. While many team members referred to the purpose of the Inquiry Team as "improving the school" or "taking what they learn with the target population schoolwide," many did not shift to this focus until late spring.

It may be that given the start-up costs of the first year of citywide implementationnamely a shortened timeframe, first year learning curves of new teams, and the need to establish teams as legitimate decision-making bodies in their schools - reaching this goal may be unrealistic. Even so, teams in about half of the schools in this sample attempted to influence their fellow teachers, administrators, and staff in the name of improving student learning, and this took some courage and conviction which was drawn from their work. The June results may lead others to hold professional development events for faculty this fall as well. Many teams felt optimistic that this goal would be reached in 2008-09.

\section{Summary}

There were some promising accomplishments during the first year of citywide implementation of the Inquiry Team process. A brief summary follows:

- By the time of the CPRE site visit in late spring, 54\% of schools had actually implemented all major stages of the Inquiry Team process. This percent rises to over $75 \%$ if we extend the implementation cycle into the early part of the 2008-09 school year, when many teams expect to conduct professional development. 
- Members generally felt the Inquiry Team process was doable given the right amount of time and support. Many teams were eager to improve and even expand their work in 2008-09.

- The study identified a set of capacities teams felt were critical to their work, which included attitudes and beliefs about the inquiry process, and skills and knowledge associated with team work and data analysis. Interviews with threequarters of teams reflected evidence that these capacities were present in various levels and distributions across team members.

- The study found evidence of strong commitment to data use, in general, and the Inquiry Team process, in particular, among a large number of the Inquiry Team members. Team commitment was associated with the opportunity to identify effective change strategies and to share "what works" with teachers. This commitment to data use also was associated with the collaborative investigation created by the inquiry process. A third of teams in this study used their outreach strategies to promote the value of data analysis and use, and to model, as a team, how data analysis can inform instruction and other school-level improvements.

- A large percent of teachers, about three-quarters, felt participation on the Inquiry Team had influenced their practice, and most were willing to continue on the team and advocate for more teacher participation.

- During the first year of citywide implementation, teams reported mixed attention to their role in stimulating school improvements. Only about half of teams in this study attempted to influence teacher practice or the wider systems that compose the school. This stage of the Inquiry Team process was the most difficult to understand and engage by teams in 2007-08. 
Formative Study of the NYC Inquiry Team Process, 2007-08 


\section{Areas of Strength and Need in 2007-08}

The study has identified some important areas for reflection by the CFI leadership and others as they prepare for the second year of the Inquiry Team process. As this report has already highlighted, there are a number of strengths to build upon from the first year of citywide implementation. These provide important levers for ratcheting up the quality and, ultimately, the impact of each Inquiry Team's work. At the same time, there are areas of the Inquiry Team process that need attention. Some of these areas may require only minor adjustments or fine-tuning. Others may require more concerted effort to garner the necessary resources and to develop the shared understandings needed to improve the quality of implementation.

\section{Areas of Strength}

The primary strengths observed by the CPRE research team include the following:

- $\quad$ Strong Momentum Going into the 2008-09 School Year.

A powerful message emanating from almost every school and found in most of the interviews was the positive anticipation about the work of the Inquiry Team in the 2008-09 school year. Almost all of the teams conveyed a strong belief that they could do an even better job with their inquiry work in the second year and were expecting to "hit the ground running" in September. A number of schools were planning to start the year off with professional development, which the teams felt would enhance the launch of their work. This kind of momentum associated with the early implementation of a new complex, large-scale initiative is unusual. If properly harnessed and directed, the anticipation and commitment voiced by Inquiry Team members should help the Department deepen the work and continue to strengthen school-based commitment to this process.

- The Establishment of the Senior Achievement Facilitator Role.

SAFs were the "face of the Inquiry Team" when they entered a school building. A majority of Inquiry Teams credited much of their professional growth, team progress, and accomplishments to the support they received from their SAFs. The shepherding of the Inquiry Team work to schools by the SAFs was critical to the progress of the schools this year, and reflected a form of differentiated coaching at its best. The positive working relationships most SAFs were able to develop with teams and principals - along with their general strategy of informing without directing — contributed to team ownership of the inquiry process and to the larger culture of empowerment. Gaps in communication did exist due to the large SAF work loads and the tendency of some principals to test out their newly found "empowerment." Creating better tools to inform the inquiry work and harnessing the resources of the SSOs will help bridge these gaps. 
- The Establishment of the Data Specialist Role.

Data Specialists provided teams with the nuts and bolts of their work - student data. It is highly unlikely that so many teams could have completed the implementation cycle this year without the continuous school-based support provided by the Data Specialists. Not only did these individuals help teams access and interpret data, they often took leadership roles in their teams, stimulating conversations or serving as the chair or chief facilitator of the team's work. Principals appeared to have selected Data Specialists wisely, but almost all of the Data Specialists required training in Department systems and some needed additional help with creating data displays to facilitate team analysis. Positions reflect priorities, and the establishment of the Data Specialist puts the use of data at the forefront in schools and proved an invaluable resource for Inquiry Teams. One concern is the volume of work expected by those holding these positions, which likely will increase as educators become more dataoriented in their practice and in their thinking about school improvement.

- The Small-Scale Elements of the Inquiry Process.

Focusing on a single problem, getting to know a small group of students, working with a small group of committed, knowledgeable educators and administrators - these were powerful design elements of the Inquiry Team process. The small elements of the design ensured that teaching and learning sat at the core of professional conversations and decision-making and that the conversations were concrete. They helped members stay focused and see the inquiry process as doable, and created personal connections at both the topic and student levels that tapped into the primary intrinsic motivation of teachers, that is, making a difference in the lives of children. As one teacher summarized her experience on the team, "I am fulfilled through the success of my students." In this respect, the purposes of the Inquiry Team process overlaps with the reasons teachers most often give for entering the teaching profession.

- Positive, Collaborative Team Dynamics.

A large percentage of teams reported developing strong working relationships that were collaborative and highly grounded in the elements of the Inquiry Team process. This is due in part to the fact that most members were invited or volunteered to serve on the team, and had expressed an interest in or had experience with data. Principals contributed to this dynamic by providing direct support for their Inquiry Teams as regular contributing members. Diverse cross-role teams that were balanced in their composition tended to have the expertise, authority, and leadership needed to implement the Inquiry Team process effectively. These teams helped administrators understand teaching in their building, and helped teachers step out of the isolation of their classrooms to see the interconnections between their classroom practice and other instructional systems in the school.

- The Role of the Principal as Contributing Member on the Inquiry Team. A large number of Inquiry Teams were high functioning, and this was connected, in part, to the active participation of their principals who were serving on these teams. Principals not only contributed to the teams' work but also facilitated contributions by 
all team members. This model provides a strong foundation for moving forward into 2008-09 and one that can support multiple Inquiry Teams in a given school.

- Clear Alignment With Other Accountability Tools.

Amidst the swirl of changes made this year as the new support structure and accountability tools were put into place, schools reported hearing the same messages from different sources. Team members saw a close alignment between their work and the standards against which schools were being measured on the Quality Review and Progress Report. The integration of these multiple tools was a strong factor in convincing educators of the value and seriousness of the Inquiry Team process. Principals saw the connections among all of the pieces, including the Principal Performance Review (PPR), and felt their work in each area was reinforcing and guiding their work in the others. For this teacher, connections were clear and seamless.

[Being on the Inquiry Team has] became more than what I expected; [it was] a learning experience. And it's interesting how it tied into the whole Quality Review. You identify deficits in your school for Quality Review, they just gave us an additional focus of isolating the strands. It made it easier to talk about the students for Quality Review because you were already working with them. I got to really use and see data in a way I had never looked at before, especially longitudinal data to see the growth.

The further integration of the Inquiry Team process with the Quality Review next year, replacing the individual student case studies, will only strengthen these connections.

\section{Areas of Need}

The CPRE research team also noted some areas of the Inquiry Team process that need attention. These include:

- The Disconnection of Some SSOs From the Inquiry Team Process.

School support organizations have a wealth of instructional expertise and offer professional development opportunities in mathematics, English and programs for special student populations such as ELLs. Yet the SSOs have not systematically connected these resources to the work of the Inquiry Teams operating in their affiliated schools. SSOs could help teams assume a more empirical posture towards the development of changes strategies by promoting research-based practices aligned with the chosen subskills. SSOs could provide opportunities for the teams to network and share their work by making better use of their principal and Data Specialist network meetings. A strong step in this direction would be offering a line of services for the Inquiry Teams as part of their portfolio of professional development and technical-assistance offerings. 
- Teacher Membership on the Inquiry Team.

The value of having classroom teachers on the Inquiry Team is widely acknowledged by both the Department and many Inquiry Team members. Yet only $25 \%$ of team members were classroom teachers in the study's school sample in 2007-08. The ambiguity of the role of the target population teachers in the inquiry process - as targets or as members - posed both technical and political challenges for the teams. Many teachers have expressed interest in joining the teams and school leaders seem committed to supporting teacher involvement. However, this will likely require creative scheduling and shifts in resources.

- The Development and Implementation of Change Strategies.

Teams were most data-informed in selecting and diagnosing student needs in their school. They were less empirically oriented when they selected or developed change strategies to address those learning needs. This may have been due to the condensed time frame of this phase of the work. Teams seem to have received little material guidance in this regard and most had few resources to draw upon. Even when relying upon internal expertise, team members often were not able to articulate the rationale for their selection of change strategies beyond noting that it came from someone knowledgeable in the group. Teams were left largely on their own to find these strategies; and convenience, more than evidence was the predominant rationale for their selections. In addition, some teams had trouble monitoring the use of the change strategies with the target population and beyond as well. Here SAFs and networks could contribute by helping teams better conceptualize this stage - the search, articulation, and implementation of change strategies — as a data-driven process as well, one grounded in evidence of effectiveness.

- Material Guidance on the Tasks and Pace of the Inquiry Team Process. Information about the inquiry process was rolled out during the year and supporting materials often were developed just in time for teams as they reached a given stage. A number of team members were baffled at the dearth of material guidance. They wanted a timeline to help them pace their work throughout the school year and to anticipate next steps. They wanted to know what other schools were doing. The latter was seen by many as a way to gauge appropriate and effective practice, and as a means of finding points of comparison to stimulate their thinking and problemsolving. Principals and Data Specialists sometimes had access to network meetings to gain this bird's eye view, but other team members typically were more isolated. The availability of such guidance would minimize the uncertainty and frustration teams felt at various times and would, at a minimum, push their questions to a second level from "What is a target population?" to "Here are some ideas for narrowing our focus." In this role, SAFs and Network Leaders could play a matchmaking role, promoting quality Inquiry Team practices and connecting schools that could benefit from one another's experience and/or expertise.

- Support for Low-Inference Transcripts. Inquiry Team members, particularly teachers, felt the opportunity to observe other teachers helped them reflect on their own instruction in new and meaningful ways. 
This activity has tremendous potential to overcome norms of privacy, create more and richer conversations about student learning that are grounded in and by instruction, and facilitate the development of a schoolwide professional learning community. The Inquiry Team could be the vehicle for widespread use of low-inference transcripts. The observation tools and guidance on their use that were available to teams varied widely and many teams struggled with identifying protocols and standards of use. Teams need help in creating cultures of trust and respect around the use of these tools that extends beyond the immediate classroom focus of the team to the entire school. Principals and teams need access to a variety of tools and more systematic guidance in terms of the technical use of these tools and their promotion within the school.

- The Promise(s) of ARIS.

Team members and Data Specialists still hold high hopes for ARIS. Despite complaints about its accessibility, the use of passwords, the timing and utility of training, and the website's reliability and capacity, most members still believe ARIS will prove an unprecedented resource for administrators, for teams, and for teachers. Some have requested a follow-up session to the general introduction to ARIS so they can have help using this portal on specific tasks. Some were looking forward to a fuller integration of ARIS with school data. One administrator explained,

On one hand, I understand this is new implementation, so the kinks are not worked out. It is more than I expected in terms of the information our school is collecting on students. It is very informative. Unfortunately, it is not cycling back to DOE, we are having trouble connecting their database to the information we have collected to make it a full range of data. In ARIS, we were supposed to flag the students and I thought that would link to their progress, but there was no way to link their data base to the rest of the information we collected. So we have to go to ARIS and interpret that and then interpret our information separately and figure out how they relate. So it is almost like adding another step to an intense data research project. So I hope over the next year or so we see ARIS become more of a useful database rather than something we look at now if we need more information we don't have.

- $\quad$ Team Focus on Stimulating Systemic Change.

Almost all teams had members who believed the ultimate goal of the Inquiry Team process was to bring about systemic changes in their school. Yet only half were able to promote recommendations for improving instruction or policies to others in the school. In some cases this was because teams were not well established decisionmaking bodies, did not have compelling change strategies or insights from their investigations to share, or did not have time to take action. One potential issue is how teams should follow up on their recommendations once they are made and the extent to which others who receive these recommendations are accountable for due diligence in further investigation or in taking action. Overall, this aspect of the Inquiry Team work was not well developed by most teams. Among team members, there is awareness that this is a missing or undeveloped aspect of their work. 
Formative Study of the NYC Inquiry Team Process, 2007-08 


\section{Recommendations}

The following suggestions aim to strengthen the Inquiry Team process in its second year of implementation. A description of the Department's plans for expanding their support for the Inquiry Team process in the 2008-09 school year is located in Appendix F.

\section{Continue funding school-based Data Specialists and the Department Senior Achievement Facilitators (SAFs).}

The Inquiry Team process was relatively successful in its first year of citywide implementation and shows great promise as a process for improving student achievement. The SAF and Data Specialist positions were critical to the progress and accomplishments of the Inquiry Teams in this study. They also were responsible for other work in the schools that directly and indirectly supported the Inquiry Teams, such as Quality Reviews and data use in general. Data Specialists often were important links for Network Leaders and/or SAFs to stay in touch with school needs and other developments that affected the work of the Inquiry Teams. The SAF position provided just-in-time advice and other supports to teams adapted to where teams were in the process and their particular needs. The incumbents in these roles will bring a wealth of experience and know-how to the process as Inquiry Teams expand within schools in 2008-09. These aspects of the program should be continued and enhanced in the future to ensure ongoing support for the Inquiry Team process.

\section{Request that SSOs align their support and professional development to better support the work of school-based Inquiry Teams.}

Most SSOs offer services or have capacity that could speak directly to Inquiry Team needs, specifically in helping schools identify change strategies. Most teams were not aware of the offerings of their SSO because Network Leaders typically interacted with principals and because most SSO services were not explicitly connected to the work of the Inquiry Teams. In some cases, it is a question of SSOs reaching beyond principals to work with teams directly. In other cases, SSOs will need to adapt or expand their inhouse expertise to provide support. In the future, SSOs should ensure that Inquiry Teams are aware of their resources and services, perhaps through informational sessions with teams near the beginning of the school year.

\section{Develop a broad system of resources to support teams as they consider change strategies to use with their target population.}

The selection process provides an opportunity to help teachers understand why the selected strategy will likely improve student learning. This includes articulating not only the theory of action underlying a given strategy but also what is known about the evidence of its effectiveness with similar or different population of students. In some 
schools the change strategy may be simply a modification of existing practice; in others it may reflect a new strategy needed to fill a longstanding omission in the school curriculum or teachers' pedagogical repertoire. These scenarios reflect different levels of capacity within a school. In the latter case, in particular, teams would benefit from assistance in thinking through options about how to respond to the needs of the target population. The SSO staff and SAF expertise could contribute greatly by raising the level of school-based discussions in terms of the search, selection/design, implementation, and evaluation of teams' change strategies. These stages of the process present opportunities, we believe, to model data-informed inquiry. The Department could support this process by offering schools a portfolio of supports that might include a mix of the following.

- A database of experts who can help teams consider a range of change strategies. This might include SAFs and immediate network specialists, but also individuals or groups beyond the SSO that schools might contract with.

- Brief reviews of the empirical literature on the learning problem, e.g., decoding, and instructional strategies that are known to work under different conditions and/or with different student populations.

- Information and training on instructional strategies that have been shown to have large effects on achievement, such as the use of structured grouping, formative assessment, or opportunities for student voice in discussions.

- Targeted professional development for groups of teachers on effective instructional strategies addressing common instructional problems that include attention to implementation considerations.

- Critical reviews of the work of other Inquiry Teams that attempt to address similar learning challenges as a means of reflecting on the efficacy of the team's strategy.

- A database of instructional change strategies used, including material supports, e.g., lesson plans and student work examples, implementation strategy and issues confronted, monitoring strategy and student performance, and team reflections on the strengths and weaknesses of this approach.

- A database of schoolwide change strategies pursued, including recommendations made, rationale, outreach strategy, steps taken to address, and follow-up to assess its effect on teaching and learning at the school at different intervals during the year.

The basic building blocks for a coherent system of supports are available through ARIS, many SSO services, and some Web-based research databases. These need only be integrated and more closely aligned with the focus and timing of the Inquiry Team process as it unfolds in schools. The Data Specialists and SAFs present important bridges for helping schools make use of these services and for populating the databases with recent Inquiry Team experiences. 


\section{Reinforce the value of diverse teams that draw from multiple role groups in the school, namely classroom teachers, nonclassroom-based instructional staff, and administrators.}

Many teams reflected this composition, and it proved meaningful to members and stimulated a positive and productive group dynamic, particularly when coupled with a chair who focused on facilitating, not directing, the team's work.

\section{Inquiry Team chairs, Data Specialists, and external support staff should help teams anticipate problem areas at different points in the process.}

This study uncovered a number of issues that teams likely will confront. These are common enough that leadership should help teams anticipate and think through strategies for how to address them. These include the following:

- Bolstering team expertise: When the team's membership does not bring the expertise needed, teams need a strategy for expanding the team or bringing on "adjuncts" to service as critical friends and/or advisors to the work.

- Designing and implementing the change strategy: When target population teachers are not formally part of the Inquiry Team, members need a strategy for convincing them of the importance of the work and engaging them as collaborators.

- Monitoring change strategy implementation: This was an after thought for some teams. Teams need help framing the work of change strategy development and implementation with participating teachers as collaborative inquiry that is not evaluative.

- Informing faculty about the team's work: Members need help planning for communication and outreach from the start of the year. They should develop an ongoing outreach strategy that establishes and uses lines of communication between faculty and the Inquiry Team through a combination of static media (e.g., bulletin boards, newsletters) and interactive venues (e.g., lunches, open team meetings, faculty meetings).

\section{Develop an ongoing multipurpose approach to networking schools around the Inquiry Team Process.}

Teams want to see and hear about what other teams are doing to gauge their progress, pick up new ideas, and find like-minded colleagues who constitute a larger community that can provide support and information. We know that the public sharing of work brings a face-to-face accountability for teams that can raise the level of seriousness about their charge. It also can prove invigorating and affirming for their work and help them see new possibilities. For these reasons, team members should have opportunities to participate in network meetings and external support staff, such as SAFs and Network Leaders, should create these opportunities throughout the year. These opportunities 
should be carefully planned so that the messages about good practice and data-oriented decision-making are reinforced.

\section{Provide more concrete guidance about good practice related to the different decision points of the Inquiry Team process and more advice about the pace of the Inquiry Team work that will enable completion of a full cycle in a single school year.}

There is a delicate balance in providing schools with guidance in the implementation of a new initiative: Too much direction and the initiative will appear to some to be too prescriptive, undermining the possibility of school ownership and the opportunities to adapt it to fit the local context and student needs; too little guidance can lead to wide variability in implementation quality and confusion and frustration among those charged with bringing the initiative to life. The Department is well aware of these tensions. In 2007-08, the SAFs were the critical bridge for teams, bringing material supports and advice developed "just in time" to support their work. For many teams this was sufficient. Even so, there is a demand from team members for more guidance about the options, trade offs, and effective practices associated with different decision points in the Inquiry Team process. There also is a demand for guidance about different pacing scenarios to help teams complete the inquiry process in a given school year. Teams may benefit from the following:

- A narrative overview of the Inquiry Team process, including it goals, decision points, and interim accomplishments, and the team capacities needed throughout the process;

- Discussion protocols, decision trees, and/or other graphic organizers to facilitate team planning at different stages of the process;

- Pacing guidance that lays out different scenarios and resources that may support each stage of the work;

- Descriptive case studies of accomplished and struggling Inquiry Teams to help teams reflect upon their practice;

- Videos of change strategies in use or professional development sessions that capture the Inquiry Team reflections on their process, new learnings, and accomplishments.

These tools should be available to all team members, but each team should determine how and when to use them. Careful thought should go into ensuring that teams are aware of the availability of these tools through multiple venues, with reminders throughout the school year. The 2007-08 implementation experiences provide a wealth of guidance to inform the development of these tools. 


\section{Focus additional resources on strengthening the professional cultures of schools with little or no history of collaborative exchanges among teachers or between administration and faculty.}

The likelihood that the inquiry process will result in an effective change strategy and will stimulate systemic change is highly dependent on the quality of adult relationships in the school. The few schools in this study with fractious faculty relationships or which were just beginning to work towards building trust around instructional conversations struggled with how to share their work and pursue larger changes. Participants in the inquiry process in these schools were unusual individuals willing to work as a team. Often principals were already working to address issues of school culture and in the 2008-09 school year their efforts should be bolstered to ensure the Inquiry Team process takes hold. If done strategically, the Inquiry Team process could illustrate the power of collaborative work relationships focused on student learning. These schools should be identified early on and quickly supported. 
Formative Study of the NYC Inquiry Team Process, 2007-08 


\title{
Appendix A Data Collection Protocols: School Level
}

\author{
Principal Interview Protocol \\ Inquiry Team Member Protocol \\ Inquiry Team Meeting Observation Protocol
}

\section{Principal \\ Interview Protocol}

\section{*Background [1-2 minutes]}

1. How long have you been principal of this school? How long have you worked in the city? In what capacity?

*School Context and the Inquiry Team [5-7 minutes]

2. What have been the major reforms or improvement strategies at the school in the past two years and how have teachers responded? [Listen for: focus, goals, accomplishments] [If asked, focus on English and mathematics initiatives]

3. What is the professional culture (teacher relationships) of the school? [probe: openness to new ideas, collegial/isolated, veteran/novice] What structures are in place to help teachers work together and how well are they working? [probe whether team/departments/PLC and nature/variation of collaboration within the school]

*Inquiry Team Process and Membership [10 minutes]

4. How have you learned about the Inquiry Team process? What training have you received and how helpful has it been? [probe if needed: sources, focus, how long]

5. What do you see as the purpose of the Inquiry Team as you understand it? How different is it from other improvement processes that school has used?

a. What is the relationship between the Inquiry Team and other decisionmaking/improvement teams in the school?

6. How was Inquiry Team membership determined? [probe process, why particular size/composition, when started to meet] Did it change during the school year? [Why?]

a. What is your sense of the strengths and weaknesses of the team's size and composition for the Inquiry process?

7. How have other teachers viewed the work of the Inquiry Team? [alt: Do teachers view the team as having a lead role in the school's improvement?] How do you know? 


\section{* Supporting the Work of the Inquiry Team [10-15 minutes]}

8. What were the major considerations when the SSO was selected last year (spring 2007)?

9. What services have you purchased from your SSO this year and how do they relate to the Inquiry Team process? Are there services you wanted but could not access? Why not?

10. How would you describe your Network? [alt: what is a network?] How does it work? How are you working with the Network Leader or Team?

11. What do you see as the major contributions of the Network Team/Leaders to the Inquiry Team?

a. Is there more the Network should/could be doing?

b. What room do you have to negotiate with the Network? Have you? Why/why not? [probe for examples]

12. How are you working with the Senior Achievement Facilitator (SAF)? What do you see as the major contributions of the SAF to the Inquiry Team?

a. Is there more the SAF should/could be doing?

b. What room do you have to negotiate with the SAF? Have you? Why/why not? [probe for examples]

*The Inquiry Team Process [20 minutes]

[NOTE: The attached graphic of the Inquiry Team process aims to serve as a memory aid and reference point for the interview. Refer to the graphic as needed.]

13. Take a moment to look over this graphic which reflects some of the steps/stages in the Inquiry Team process. We developed this from some department materials. We want to use this graphic to talk about your team's experience with this process. First some quick questions:

a. Does this graphic reflect the process as you experienced it? If no, please explain.

b. Is there other team work not reflected in here?

14. For the next 10 minutes or so, I would like to hear about the work of the Inquiry Team this year. For each step/stage we are particularly interested in learning how the team approached it and what role you played in the work [alt: how you were involved]. Let's start with Step A. "Determining a school-wide focus." [If participant does not move through steps, prompt: "how about the next step?"]

NOTE: Probe for examples at individual steps-as appropriate. For steps skipped ask "why?" For steps not reached, ask what the team hopes to accomplish and any strategy that has been discussed. 


\section{*Taking the Work School-Wide [7-10 minutes]}

[if not addressed in Q14 or need to extend]

15. In general, how have other teachers learned about the work of the Inquiry Team? What part of the work have teachers been most interested in?

16. What kind of school-wide changes has the Inquiry Team pursued and how have teachers responded? [Listen for and probe as needed for (a) change in instructional practices; (b) change in school-level routines; or (c) change in the use of data.]

a. What do you see facilitating this work?

b. What, if anything, has been getting in the way?

*Reflections on the Inquiry Team Process [3-5 minutes]

17. Is the Inquiry Team process worthwhile for your school? Please explain.

a. What could be done to make the process more valuable for your school?

18. What are your plans for the Inquiry Team process next year and what do you hope to accomplish?

\section{*Closing}

19. Is there anything more to add about your experience with the Inquiry Team process?

20. Is it possible to get a copy of the minutes of the Inquiry Team meetings? Can we pick-up today?

[end] 


\section{Inquiry Team Member \\ Interview Protocol}

*Background [2 minutes]

1. What is your role(s) in this school? How many years have you been teaching and how long at this school?

[Data analyst: How does this role compare to others you have had in the past?]

*Inquiry Team Membership [5-7 minutes]

2. What is the purpose and work of the Inquiry Team as you understand it? Have you done anything like this before? [e.g., data analysis, leadership team, designing change strategies, monitoring small groups of students] Please explain. [listen for: in general or at the school]

3. How did you come to join the school's Inquiry Team? Is it what you expected? Please explain briefly.

[Data analyst: What is your role on the team? What kinds of tasks have you been doing for the team?]

4. What training have you received as a member and how helpful has it been? [probe if needed: source, focus, duration]

[Data analyst: What additional training or support have you received? What was the most helpful? Why?]

*The Work of the Inquiry Team [15-20 minutes]

[NOTE: The attached 5-step graphic of the Inquiry Team process aims to serve as a memory aid and reference point for the interview. Refer to the graphic as needed. For use with questions 5-12]

5. [Hand to participant] Take a moment to look over this graphic which reflects some of the steps/stages in the Inquiry Team process. We developed this from some department materials. We want to use this graphic to talk about your team's experience with this process. First some quick questions:

a. Which steps has the team had a chance to work through?

b. Is there other team work not reflected on this page?

c. Which steps did the team spend the most time on? The least time?

6. For the next 10 minutes or so, we would like to hear about the work of the Inquiry Team this year. For each step/stage we are particularly interested in learning how the team approached it and what role you played in the work [alt: how you were involved]. Let's start with Step A. "Determining a school-wide focus" [also listed on graphic]. [if participant does not move through steps, prompt: "how about the next step?"]

NOTE: Probe for examples at individual steps, as appropriate. For steps skipped ask "why?" For steps not reached, ask what the team hopes to accomplish and any strategy that has been discussed. 
[It not answered in Q6 above, probe on the "use of data"' to extend]

7. What was the quality of data/information the team had access to? How well was the team able to explore the data?

a. What do you see facilitating the team's use of data? [probe for examples]

b. What, if anything, has proven an obstacle? [probe for examples]

[Note potential data include: state date, periodic assessments, quality reviews, progress reports, other, OR evidence of effectiveness of different change strategies OR teacher experiences-"wisdom of practice" either within the school or beyond]

[It not answered in Q6 or Q7 above, probe on "challenges or issues" to extend]

8. Generally, what challenges or issues emerged for the team during the Inquiry Team process and how were they resolved?

[Probe as needed] How did that impact what you wanted to accomplish?

*Supporting the Work of the Inquiry Team [7-10 minutes]

9. At different steps of the process, how were some team member(s) particularly important or influential to the team's decisions? Please describe.

10. What role did the principal take in the Inquiry Team process? From your perspective, how did that influence the team's focus and decisions? Please provide an example or two.

11. How has Network staff been involved in the Inquiry Team? [probe if needed for examples]

If involved:

a. For which tasks/steps was the Network Staff most critical? How so?

b. How might someone in that role best support your team?

12. How has the Senior Achievement Facilitator (SAF) been involved in the Inquiry Team? [probe if needed for examples]

If involved:

a. For which tasks/steps was the SAF most critical? How so?

b. How might someone in that role best support your team?

*Taking the Work School-Wide [4-10 minutes]

[if not addressed in Q6 or need to extend]

13. In general, how have other teachers learned about the work of the Inquiry Team? What part of the work have teachers been most interested in?

14. What kind of school-wide changes has the Inquiry Team pursued and how have teachers responded? [Listen for and probe as needed for (a) change in instructional practices; (b) change in school-level routines; or (c) change in the use of data.]

a. What do you see facilitating this work?

b. What has been getting in the way? 
*Reflections on the Inquiry Team Process [2-4 min]

15. Is the Inquiry Team process worthwhile for your school? Please explain.

a. Would you consider serving again on the team next year? Why or why not?

16. How might the Inquiry Team process be more valuable to your school next year? In general? [Alt: how might the process be improved for your school?]

*Closing

17. Those are all of our questions. Is there anything more to add about your experience as an Inquiry Team member?

School artifacts to search for:

- Inquiry team minutes [ask principal]

- Inquiry team produced materials related to data analysis, the change strategy, teacher outreach, student monitoring, etc.

[end] 


\section{Inquiry Team Meeting Observation Protocol}

\section{CPRE Observer:}

\section{Background Information}
A. School Name:
B. SSO Affiliation:
C. Date/Time:
D. Regular Time or Adjusted for CPRE?
E. Number Participating:
F. List Members Present (w/titles \& roles):
G. Members Absent? Reason?:
H. Who Chaired/Facilitated the Meeting?:
I. Was an Agenda (written or verbal) Used? Yes/No If so, Available in CPRE File?
Yes/No
J. Meeting Focus (short summary):

K. Anything else important to note in order to understand what happened? Please explain.

\section{Meeting Summary}

A. Describe the focus of the meeting conversation and any problems/issues discussed (please write with as much detail as possible and use as much space as needed):

B. How was student data or evidence of effectiveness used?

C. What decisions were discussed? What was decided?

D. Role of external support staff (e.g., SSO, network staff, SAF, other), if any? What did the support staff contribute to the discussion? How did others respond to the external support staff?

E. Role of the principal, if any? What did the principal contribute to the discussion? How did others respond to the principal?

F. To what extent did the team discussion suggest they were taking a leadership role in the school? Please explain.

G. Which team member(s) appeared most critical or central to the work of the team during this meeting and why? How did others respond to this member(s)? 
Please rate the team meeting on a variety of dimensions using the 5 point scale. Justify each rating with examples from your notes. Where there is uncertainty, explain why.

$H$. Overall, to what extent did the observed discussion reflect an understanding of the Inquiry Team process? RATING:

1

Continuous

Confusion:

Persistent questions

about the purpose

and goals of the

meeting/task.
2

Task Oriented:

Surface understanding,

oriented towards

completing the task.
4

5

Deep Understanding: Strong sense of the task's purpose and its connection to other aspects of the Inquiry Team process, instructional practice, and school structure.

Rating Justification:

I. What was the professional tone of the meeting? RATING:

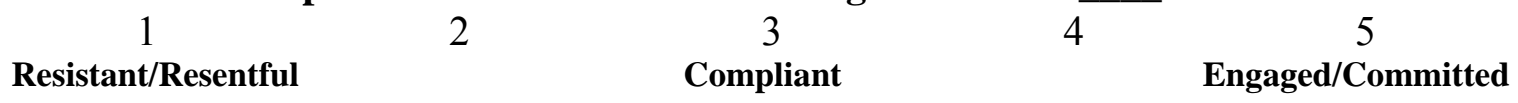

Rating Justification:

J. To what extent was there an established routine to the meeting such that the nature of the discussion (e.g., topic, format, or decisions) seemed familiar to members and roles well-established? RATING:
1
3
4
2

Confusion/Uncertainty

$\underline{\text { Rating Justification: }}$

K. What was the level of participation across members? RATING:
1
2
3
4 5
Low:
Broad Participation:
Each member
contributing in small and large ways.

Little to no participation;

one voice dominates.

$\underline{\text { Rating Justification [be sure to describe dominant or silent voices]: }}$

L. What was the quality of team member relationships? RATING:

$\begin{array}{ccccc}1 & 2 & 3 & 4 & 5 \\ \text { Sterile/Disrespectful } & & & & \text { Warm/Respectful }\end{array}$

Rating Justification:

\section{Running Record of Meeting}

This section contains your notes from the meeting. If possible note administrator (A), teacher/coaches $(\mathrm{T})$, and/or external support staff $(\mathrm{S})$ comments. Track Data Specialist contributions if possible. Add descriptions of any important interactions to understand the group dynamic. If appropriate, request copies of documents referenced. 


\section{Appendix B: \\ Data Collection Protocols: External Support Structure

\author{
School Support Organization Leadership Protocol \\ Network Leader Protocol \\ Senior Achievement Facilitator (SAF) Protocol
}

\section{School Support Organization (SSO) Leadership \\ Interview Protocol}

\section{*Background [2-3 minutes]}

1. When did you join the organization and what first attracted you?

2. Please describe your role and responsibilities at the SSO?

*SSO Portfolio of Work [10 minutes]

3. What is the mission of SSO today and how has it changed over time? [probe: What have been the driving forces?]

4. What would you consider the core capacities of this organization? [alt: What do you do well or have the most experience with?] How are you looking to expand in terms of services?

5. Where is your organization working [clients/regions] and in what capacity? Are you working in partnership with other organizations? [probe: locally or beyond] How are you looking to expand in terms of clients? [probe: locally or beyond]

6. What are the characteristics of the schools that tend to benefit most from your services?

*SSO in New York [20 minutes]

7. Why apply/seek to become a NYC SSO?

a. [skip if covered by Q5:] What experience does the SSO have working in the city?

8. What background do you look for in your staff who work directly with schools?

9. What are some reasons NYC schools have selected your organization? [alt: What have you heard?] How has that influenced your work or your staff's?

10. Please describe the different types of services you offer to NYC schools.

Probe:

a. Are they packaged together or can schools purchase each separately, ala carte?

b. How do they differ in costs? 
11. How different are the service agreements across your NYC schools?

Probe:

a. Which are most popular and why?

b. Are there services you would like to see schools purchasing more? Why? What is getting in the way?

*SSO and Inquiry Team Process [25 minutes]

12. How similar is the Inquiry Team process to anything you have been doing with schools previously?

13. What do you see as the strengths and weaknesses of the Inquiry Team process?

a. Have you tried to influence the design of this process? [Why not? or How and with what success?]

14. What types of training have you or others at the SSO received regarding the Inquiry Team process and how sufficient has this been?

15. Which of your services or products is directly supporting the Inquiry Team process? Which are new and which were adapted from existing services/products you were providing?

16. Have you had to make any midcourse adjustments this year in terms of your products or services supporting the Inquiry Team? [probe: what did and what responding to]

a. Do you anticipate any changes in your services for next year? If so, what are you planning and why?

17. How are you working with the Lead Senior Achievement Facilitator? What have been the advantages and disadvantages of this arrangement?

18. What expectations do you think the Department holds for your work? [probe which office interact with the most] What are you being held accountable for? Has this changed over the year?

*Closing [1-2 minutes]

19. Those are all our questions, is there anything more you would like to add about supporting the Inquiry Team process?

Documents to request:

- List of services offered/cost

- Examples of Inquiry Team support materials/tools used with schools

[end] 


\section{Network Leader/Staff \\ Interview Protocol}

\section{*Background [3-5 minutes]}

1. How did you become a Network Leader and what attracted you to the position?

2. Please describe your role and responsibilities as a Network Leader? How does this role compare to others you have had in the past?

*Working with Network Schools [10 minutes]

3. Tell me about your Network. [Probe: size, school type, location, variation in performance.] How does this network compare to other networks working with this SSO?

4. What are some reasons your schools selected this SSO? [alt: What have you heard?] How has this influenced your work?

5. How different are your service agreements with schools across the network [probe different levels/costs]? How has this influenced your work?

a. Are there services you want to see schools purchasing more? Why and what is getting in the way? [alt: what could they do then?]

6. What is the role of the Network in the Inquiry Team process?

a. How are you working with the SAF? [probe: What is the division of labor in working with schools] What have been the advantages and disadvantages of this arrangement?

\section{*Views on the Inquiry Team Process [10 minutes]}

7. When did you first hear about the Inquiry Team process? How similar was it to anything you had been doing with schools?

8. What do you see as the strengths and weaknesses of the Inquiry Team process?

a. Have you tried to influence the design of this process? [if so, how and with what success? Or Why not?]

9. What kind of training have you received regarding the Inquiry Team process and how sufficient was this? How have you used those trainings?

\section{*Supporting the Inquiry Team Process [10 minutes]}

10. In thinking about your schools, what has been your approach to supporting the Inquiry Team process? Probe:

a. How do you decide which schools to work with and what to do?

b. To what extent have you customized your approach for different schools? Why?

11. What do you think the principal's role should be in the Inquiry Team process? How does that compare to the roles principals are taking? How are you working with them?

12. [Hand graphic to participant.] Here is a graphic of the steps or stages in the Inquiry Team process. We developed this from some department materials.

a. Does this capture the process as you have come to experience it? How so? 
Thinking broadly about the schools you are responsible for:

b. Which steps have teams engaged most successfully? In what ways are you seeing them grow? [e.g., new capacities, new insights, new commitments] What do you see facilitating this process?

c. Which steps proved most challenging for teams/schools? [alt: which struggle to understand or implement?] What were the main obstacles? How did this affect their experience with the process?

\section{*Work in Participating Schools [10-15 minutes each school]}

13. Here is a report of the Inquiry Team at School A.

a. Briefly, how were you involved in the Inquiry Team at School A?

b. Was there a time when you had to intervene because the team was in trouble or was headed in a direction that seemed ineffective? Please explain. What happened?

i. How comfortable did you feel in that role? Why?

c. How effective was the team in making use of student data?

i. What decisions did the team make based on this information and to what extent were those decisions warranted by the data? Why or why not? [probe: if took actions to endorse or change course]

d. Which team member(s) were particularly influential in the team's discussions? How so? [Alt: Are there particular products or outcomes that would have been different if they were not involved? Please explain.]

e. How was the principal involved? How did this involvement influence the process?

f. In what ways has this team/school grown through this process? [e.g., new capacities, new insights, new commitments] What were the main achievements of this team?

g. [Skip if address in 13b] What were the major challenges they faced, if any? How were they resolved?

h. Looking towards next year, what are your recommendations for this school about next steps with the Inquiry Team process? In what areas will they need extra support?

NOTE: repeat set of questions for each school in the study.

\section{*Reflections on the Inquiry Team Process [5 minutes]}

14. Is the Inquiry Team process worthwhile for your schools? How so? What could be done to make the process be more valuable to your schools?

\section{*Closing}

15. Those are all our questions. Is there anything more you would like to add about working with schools on the Inquiry Team process?

[end] 


\section{Senior Achievement Facilitator (SAF) Interview Protocol}

\section{*Background [5 minutes]}

1. How did you become a Senior Achievement Facilitator (SAF) and what attracted you to the position?

2. Please describe your role and responsibilities as a SAF? How does this role compare to others you have had in the past?

3. What expectations do you think the Department holds for your work? What are you being held accountable for? Has this changed over the year?

*Views on the Inquiry Team Process $[10$ minutes $]$

4. How similar/different is the Inquiry Team process to anything you had been doing with schools in the past?

5. What do you see as the strengths and weaknesses of the Inquiry Team process?

a. Have you tried to influence the design of this process? [if so, how and with what success? Or Why not?]

6. What kind of training have you received regarding the Inquiry Team process and how sufficient was this? How have you used those trainings?

* Supporting the Inquiry Team Process [10-15 minutes]

7. In thinking about your schools, what has been your approach to supporting the Inquiry Team process? Probe:

a. How do you decide which schools to work with and what to do?

b. To what extent have you customized your approach for different schools? Why?

8. What is the role of the Network leaders/teams in the Inquiry Team process and how are you working with them? [probe: What is the division of labor in working with schools? How evolved during the year?]

a. What have been the advantages and disadvantages of this arrangement? [probe: issues arose and how resolved?

9. What do you think the principal's role should be in the Inquiry Team process? How does that compare to the roles principals are taking? How are you working with them?

10. Here is a graphic of the steps or stages in the Inquiry Team process. We developed this from some department materials.

a. Does this capture the process as you have come to experience it? How so?

Thinking broadly about the schools you are responsible for:

b. Which steps have teams engaged most successfully? In what ways are you seeing them grow? [e.g., new capacities, new insights, new commitments] What do you see facilitating this process?

c. Which steps proved most challenging for teams/schools? [alt: which struggle to understand or implement?] What were the main obstacles? How did this affect their experience with the process? 
*Work in Participating Schools [10-15 minutes each school]

11. Here is a report of the Inquiry Team at School A.

a. Briefly, how were you involved in the Inquiry Team at School A?

b. Was there a time when you had to intervene because the team was in trouble or was headed in a direction that seemed ineffective? Please explain. What happened?

i. How comfortable did you feel in that role? Why?

c. How effective was the team in making use of student data?

i. What decisions did the team make based on this information and to what extent were those decisions warranted by the data? Why or why not? [probe: if took actions to endorse or change course]

d. Which team member(s) were particularly influential in the team's discussions? How so? [Alt: Are there particular products or outcomes that would have been different if they were not involved? Please explain.]

e. How was the principal involved? How did this involvement influence the process?

f. In what ways has this team/school grown through this process? [e.g., new capacities, new insights, new commitments] What were the main achievements of this team?

g. [Skip if address in 11b] What were the major challenges they faced, if any? How were they resolved?

h. Looking towards next year, what are your recommendations for this school about next steps with the Inquiry Team process? In what areas will they need extra support?

NOTE: repeat set of questions for each school in the study.

*Reflections on the Inquiry Team Process [5 minutes]

12. Is the Inquiry Team process worthwhile for your schools? How so? What could be done to make the process more valuable to your schools?

\section{*Closing}

13. Those are all our questions. Is there anything more you would like to add about working with schools on the Inquiry Team process?

Documents to request:

- Examples of Inquiry Team support materials/tools used with schools

[end] 


\section{Appendix C: \\ School Profile Data Summary \\ Template \\ School Profile \\ Data Summary Template}

CPRE Identifier:

SSO:

Network Leader:

SAF:

Quality Review Score:

Progress Report Grade:

School Level:

CPRE Researcher(s):

\section{SCHOOL BACKGROUND/CONTEXT}

Demographics

- Principal Status: [length of service at school \& if new to profession]

- School Size (student/faculty):

- Race/Ethnicity: African-American (\%), Asian (\%), Hispanic (\%), Caucasian (\%), Native American/Pacific Islander (\%), Other ( \%)

- Poverty: (\%free/reduced price lunch):

- Borough:

Prior Improvement Efforts:

Professional Culture:

Notable Events/Characteristics: [may include recent events or change in faculty or climate of the visits, etc.]

\section{INQUIRY TEAM MEMBERS}

\# Members:

Core Members (roles/titles): [list by role(s) and titles. Note team chair and Data Specialist also.] Informal/infrequent members (roles, titles) and explain: [For example, the principal in some schools attends sometimes, but not always.]

\section{SITE VISIT DATA COLLECTED}

Interviews (note omissions):

Observation (duration/time): [note if not conducted and reasons]

Documents collected (list):

\section{SUMMARY INFORMATION}

*Where in process was the team at the time of the CPRE visit?

*What stage did the team anticipate reaching by the end of the school year?

*At which stage did the team spend the most time?

*At which stage did the team spend the least time? 


\section{THE WORK OF THE INQUIRY TEAM}

A. Team Composition and Dynamics:

B. Approach To The Inquiry Team Process:

- Finding A Problem

- Developing And Evaluating The Effectiveness Of Solutions

C. The Quality Of Data Used And Analysis To Support Key Decisions

D. Principal's Role And Its Influence On The Team's Work

E. Factors That Seem To Be Facilitating Or Inhibiting The Team's Work

VI. THE INQUIRY TEAM AS A CHANGE AGENT WITHIN THE SCHOOL

A. Team's View Of Its Role In The School

B. Quality of the Team's Approach to Informing Others about Their Work and How

Teachers Responded

C. Identifying and Pursuing Changes to Improve Student Learning

D. To What Extent Is The Team's Change Strategy Sustainable

E. Factors That Seem To Facilitate Or Inhibit the Team's Effort To Stimulate Other/Broader Changes To Improve Student Learning.

VII. SOURCE AND QUALITY OF SUPPORT

A. Type Of Training Received And Perceived Adequacy

B. Nature Of Individual Team Member Contributions To The Team At Different Stages Of The Inquiry Team Process

C. SAF Role And Contributions

D. Network Leader/Staff Role And Contributions

E. How Principal/Team Made Use Of External Support

VIII. PERCEIVED VALUE OF THE INQUIRY TEAM PROCESS

A. Team Member Views On The Value Of The Process

B. Principal Views On The Value Of The Process

C. Ideas About How The Process Could Be More Valuable To The School

D. Plans/Intentions/Hopes for Next School Year (2008-2009SY) 


\section{Appendix D Inquiry Team Composition}

\begin{tabular}{|c|c|c|c|c|c|c|c|}
\hline \multicolumn{8}{|c|}{ Inquiry Team Composition and Size in 41 Study Schools, 2007-208 } \\
\hline School & Principal & $\begin{array}{l}\text { Assistant } \\
\text { Principal }\end{array}$ & $\begin{array}{l}\text { Data } \\
\text { Specialist }\end{array}$ & $\begin{array}{l}\text { Classroom } \\
\text { Teacher }\end{array}$ & $\begin{array}{l}\text { Non-Classroom } \\
\text { Based }\end{array}$ & Other & Total \\
\hline 1 & 1 & & $1(\mathrm{AP})$ & $\begin{array}{l}14^{\text {th }} \text { gr. } \\
13^{\text {rd }} \text { gr. Bilingual }\end{array}$ & 1 IEP Teacher & $\begin{array}{l}1 \text { Social } \\
\text { Worker }\end{array}$ & 6 \\
\hline 2 & 1 & & $\begin{array}{l}1 \text { (Duties } \\
\text { across } 2 \text { APs; } \\
\text { one on team) }\end{array}$ & $13^{\text {rd }}$ gr. ESL & $\begin{array}{l}1 \text { AIS Teacher } \\
1 \text { ESL Teacher }\end{array}$ & & 5 \\
\hline 3 & 1 & & $\begin{array}{l}1 \text { (SPED } \\
\text { Tchr) }\end{array}$ & $110^{\text {th }}$ gr English & 1 ESL Teacher & & 4 \\
\hline 4 & 1 & & $1(\mathrm{AP})$ & $\begin{array}{l}1 \text { 10th gr. Math } \\
19 \text { th gr. English } \\
19 \text { th gr. Soc Studies }\end{array}$ & & & 5 \\
\hline 5 & 1 & 1 & $\begin{array}{l}\text { (Test } \\
\text { Crdntr ) }\end{array}$ & & $\begin{array}{l}2 \text { Literacy Coaches } \\
1 \text { Math Coach } \\
1 \text { ESL teacher } \\
1 \text { Early Gr. } \\
\text { Reduction Teacher }\end{array}$ & & 8 \\
\hline 6 & & $\begin{array}{l}1 \mathrm{~K}-1 / \mathrm{SC} \text { crd } \\
1 \mathrm{ELL} / \mathrm{test} \mathrm{cr}\end{array}$ & 1 (AP 2-3) & $15^{\text {th }}$ gr. ESL & $\begin{array}{l}1 \text { Math Coach } \\
1 \text { Literacy Coach } \\
1 \text { ESL Teacher }\end{array}$ & & 7 \\
\hline 7 & 1 & & $\begin{array}{l}1 \text { (AP test } \\
\text { Crdtr) }\end{array}$ & $\begin{array}{l}14^{\text {th }} \text { gr. } \\
15^{\text {th }} \text { gr. }\end{array}$ & 1 Tech Teacher & & 5 \\
\hline 8 & & $\begin{array}{l}1 \text { SPED, } \\
\text { gr.4-6 }\end{array}$ & $\begin{array}{l}2 \text { (test } \\
\text { Crdntr) }\end{array}$ & 14 th gr. & 1 Literacy Coach & & 5 \\
\hline 9 & 1 & & $\begin{array}{l}1 \text { (AIS } \\
\text { Team Ldr) }\end{array}$ & $\begin{array}{l}14^{\text {th }} \text { gr. } \\
15^{\text {th }} \text { gr. }\end{array}$ & $\begin{array}{l}1 \text { Tech } \\
\text { Tchr/Budget } \\
\text { Manager } \\
1 \text { Reading Coach } \\
\end{array}$ & & 6 \\
\hline 10 & 1 & 1 gr. $3-5$ & $\begin{array}{l}1 \text { (Literacy } \\
\text { Coach, AIS } \\
\text { Crdntr) }\end{array}$ & $\begin{array}{l}1 \text { 5th gr. } \\
1 \text { ESL } \\
14 \text { th gr. }\end{array}$ & 1 Math Coach & $\begin{array}{l}1 \text { Social } \\
\text { Worker }\end{array}$ & 8 \\
\hline 11 & 1 & 1 & $\begin{array}{l}1(3-5 \\
\text { Literacy } \\
\text { Coach })\end{array}$ & $\begin{array}{l}14^{\text {th }} \text { gr. } \\
14 \text { th gr/gr leader }\end{array}$ & $\begin{array}{l}1 \text { Literacy Coach } \\
1 \text { Math Coach }\end{array}$ & & 7 \\
\hline 12 & 1 & & $\begin{array}{l}1 \text { (Tech. } \\
\text { Crdntr } \\
\text { /Busnss } \\
\text { Mngr) }\end{array}$ & & $\begin{array}{l}1 \text { Math Coach } \\
1 \text { Literacy Coach }\end{array}$ & $\begin{array}{l}1 \text { Bilngl } \\
\text { Crdntr } \\
1 \text { TestCrdntr }\end{array}$ & 6 \\
\hline 13 & 1 & $\begin{array}{l}1 \text { gr. K-2 } \\
1 \text { gr } 3-5\end{array}$ & 1 & & $\begin{array}{l}2 \text { ELA Coaches } \\
1 \text { Math Coach/4th } \\
\text { gr. AIS }\end{array}$ & & 7 \\
\hline 14 & 1 & $\begin{array}{l}1 \text { gr. } 4 \\
1 \text { gr. } 5 / \mathrm{K}-1\end{array}$ & $\begin{array}{l}1 \text { (Math } \\
\text { Coach) }\end{array}$ & $12^{\text {nd }}$ gr. & $\begin{array}{l}2 \text { Literacy Coaches } \\
1 \text { Tech. Teacher } \\
1 \text { AIS Teacher } \\
1 \text { ELL/ESL Spclst } \\
1 \text { Math Coach }\end{array}$ & & 11 \\
\hline
\end{tabular}




\begin{tabular}{|c|c|c|c|c|c|c|c|}
\hline \multicolumn{8}{|c|}{ Inquiry Team Composition and Size in 41 Study Schools, 2007-208 } \\
\hline School & Principal & $\begin{array}{l}\text { Assistant } \\
\text { Principal }\end{array}$ & $\begin{array}{l}\text { Data } \\
\text { Specialist }\end{array}$ & $\begin{array}{l}\text { Classroom } \\
\text { Teacher }\end{array}$ & $\begin{array}{l}\text { Non-Classroom } \\
\text { Based }\end{array}$ & Other & Total \\
\hline 15 & 1 & 1 & $\begin{array}{l}\text { (Test } \\
\text { Crdntr ) }\end{array}$ & $\begin{array}{l}1 \text { Music/Arts } \\
\text { Crdntr } \\
1 \text { 4th gr. } \\
1 \text { 5th gr./gifted } \\
1 \text { 5th gr./SPED/ } \\
\text { schl ldrshp chr }\end{array}$ & $\begin{array}{l}1 \text { Math Coach } \\
1 \text { Literacy Coach }\end{array}$ & & 9 \\
\hline 16 & 1 & 1 & $\begin{array}{l}1 \text { (Literacy } \\
\text { Coach) }\end{array}$ & $\begin{array}{l}14^{\text {th }} \text { gr. } \\
1 \text { 3rd gr./Math Coach }\end{array}$ & $\begin{array}{l}1 \text { Reading First } \\
\text { Coach }\end{array}$ & & 6 \\
\hline 17 & & $\begin{array}{l}1 \text { Math } \\
1 \text { SocStud } \\
1 \text { Science }\end{array}$ & $\begin{array}{l}1 \text { (AP } \\
\text { prgrming) }\end{array}$ & $\begin{array}{l}1 \text { 10th gr. English } \\
1 \text { 10th gr. Math } \\
1 \text { 9th gr. Soc Studies }\end{array}$ & & & 8 \\
\hline 18 & 1 & $1 \mathrm{pK}-2$, AIS & $\begin{array}{l}1 \text { (Math } \\
\text { Coach) }\end{array}$ & & $\begin{array}{l}1 \text { Literacy Coach } \\
1 \text { AIS team leader }\end{array}$ & $\begin{array}{l}1 \text { Librarian/ } \\
\text { Testing } \\
\text { Crdntr }\end{array}$ & 6 \\
\hline 19 & & & $\begin{array}{l}1 \text { (Tech. } \\
\text { Crdntr) }\end{array}$ & 1 math & 1 AIS Teacher & & 3 \\
\hline 20 & 1 & $1 \mathrm{pK}-1$ & $1(\mathrm{AP})$ & $\begin{array}{l}13^{\text {rd }} \mathrm{gr} . \\
14^{\text {th }} \mathrm{gr} . \\
15^{\text {th }} \mathrm{gr} .\end{array}$ & $\begin{array}{l}1 \text { Reading Tchr } \\
1 \text { Literacy Coach, } \\
\text { Test Crdntr }\end{array}$ & $\begin{array}{l}4 \\
\text { Consultants }\end{array}$ & 12 \\
\hline 21 & 1 & 1 test coord & $\begin{array}{l}\text { (Math } \\
\text { Staff } \\
\text { Developer) }\end{array}$ & & $\begin{array}{l}1 \text { Literacy Staff } \\
\text { Developer } \\
3 \text { AIS K-1, 2-3,4-5 } \\
1 \text { AIS Literacy 6-8 } \\
1 \text { AIS Math 6-8 }\end{array}$ & & 9 \\
\hline 22 & 1 & $\begin{array}{l}1 \mathrm{pK}-2 \\
1 \text { gr. 3-5, } \\
\text { SPED/ ESL }\end{array}$ & $\begin{array}{l}1 \text { (IEP } \\
\text { Tchr) }\end{array}$ & $13^{\text {rd }}$ gr. & $\begin{array}{l}1 \text { Math Coach } \\
1 \text { Literacy Coach }\end{array}$ & & 7 \\
\hline 23 & 1 & & 1 & $\begin{array}{l}15 \text { th gr. } \\
18 \text { th gr. }\end{array}$ & 1 AIS Crdntr & & 5 \\
\hline 24 & 1 & & $1(\mathrm{AP})$ & & $\begin{array}{l}1 \text { Math Coach } \\
1 \text { Literacy Coach }\end{array}$ & & 4 \\
\hline 25 & & 1 & $\begin{array}{l}1 \text { (Math } \\
\text { Coach) }\end{array}$ & & $\begin{array}{l}1 \mathrm{IEP} / \mathrm{AIS} \mathrm{Tchr} \\
1 \mathrm{Read} 180 \mathrm{Tchr}\end{array}$ & & 4 \\
\hline 26 & 1 & 2 & & $\begin{array}{l}1 \text { SPED } \\
2 \text { Social Studies }\end{array}$ & 1 Literacy Coach & & 7 \\
\hline 27 & 1 & $\begin{array}{l}1 \\
\text { Supervision }\end{array}$ & $\begin{array}{l}1 \text { (AP } \\
\text { SPED, } \\
\text { SocSt, ESL, } \\
\text { Guidance) }\end{array}$ & $\begin{array}{l}1 \text { Business } \\
1 \text { SPED } \\
1 \text { Math } \\
1 \text { Math (gr.9/12) }\end{array}$ & & & 7 \\
\hline 28 & 1 & & $\begin{array}{l}1 \text { (SEM } \\
\text { Crdntr ) }\end{array}$ & $\begin{array}{l}17^{\text {th }} \text { gr. ELA } \\
18^{\text {th }} \text { gr. ELA } \\
14 \text { th gr. (UFT } \\
\text { Chapter Leader) } \\
14 \text { th/5th gr. }\end{array}$ & $\begin{array}{l}\text { 1 Math Coach } \\
1 \text { Literacy Coach } \\
1 \text { Reading Rcvry } \\
1 \text { Literacy } \\
\text { Coach/Consultant }\end{array}$ & & 6 \\
\hline 29 & 1 & & $1(\mathrm{AP})$ & 15 th gr. & 1ESL teacher & & 8 \\
\hline 30 & 1 & & $\begin{array}{l}\text { 1(Tchr } \\
\text { Mentor) }\end{array}$ & $\begin{array}{l}13^{\text {rd }} \text { gr } \\
14^{\text {th }} \text { gr. SPED }\end{array}$ & & & 4 \\
\hline 31 & 1 & & $1(\mathrm{AP})$ & 2 3rd gr. & 1 Literacy Coach & $\begin{array}{l}\text { 1 Parent } \\
\text { Crdntr } \\
1 \text { Occptinl }\end{array}$ & 7 \\
\hline
\end{tabular}




\begin{tabular}{|c|c|c|c|c|c|c|c|}
\hline \multicolumn{8}{|c|}{ Inquiry Team Composition and Size in 41 Study Schools, 2007-208 } \\
\hline School & Principal & $\begin{array}{l}\text { Assistant } \\
\text { Principal }\end{array}$ & $\begin{array}{l}\text { Data } \\
\text { Specialist }\end{array}$ & $\begin{array}{l}\text { Classroom } \\
\text { Teacher }\end{array}$ & $\begin{array}{l}\text { Non-Classroom } \\
\text { Based }\end{array}$ & Other & Total \\
\hline & & & & & & Therapist & \\
\hline 32 & 1 & & $\begin{array}{l}\text { 1(8th gr. } \\
\text { Math Tchr) }\end{array}$ & $\begin{array}{l}17 \text { th gr. Math } \\
24 \text { th gr. }\end{array}$ & $\begin{array}{l}1 \text { IEP/Reading } \\
\text { Recovery Teacher }\end{array}$ & & 6 \\
\hline 33 & & $\begin{array}{l}1 \mathrm{ESL} / \mathrm{Sc} / \\
\mathrm{Blngl} / \mathrm{PE}\end{array}$ & $\begin{array}{l}\text { (AP, } \\
\text { Testing } \\
\text { Crdntr) }\end{array}$ & $\begin{array}{l}1 \text { 6th gr. Math } \\
17 \text { th gr. Math }\end{array}$ & & $\begin{array}{l}1 \text { asst admin. } \\
1 \mathrm{GC}\end{array}$ & 6 \\
\hline 34 & & & $1(\mathrm{AP})$ & 1 Tech \& Math & $\begin{array}{l}1 \text { AIS Teacher } \\
1 \text { Staff Developer }\end{array}$ & & 4 \\
\hline 35 & & 1 & $\begin{array}{l}1 \text { (9th \&10th } \\
\text { gr. Social } \\
\text { Studies } \\
\text { Teacher) }\end{array}$ & $\begin{array}{l}111 / 12 \text { th gr. Social } \\
\text { Studies }\end{array}$ & & & 3 \\
\hline 36 & 1 & $\begin{array}{l}1 \text { Guidance } \\
1 \text { Math }\end{array}$ & $\begin{array}{l}1 \text { Consultant } \\
1 \text { Math Tchr }\end{array}$ & & 1 SPED Tchr/UFT & & 6 \\
\hline 37 & 1 & 1 Curr \&Instr & $\begin{array}{l}1 \text { (Soc Studie } \\
\text { Science Tchr) }\end{array}$ & & $\begin{array}{l}1 \text { IEP Teacher } \\
1 \text { Math Coach }\end{array}$ & & 5 \\
\hline 38 & & & $1(\mathrm{AP})$ & 1 3rd gr. & $\begin{array}{l}1 \text { AIS Teacher } \\
1 \text { Literacy Coach }\end{array}$ & $1 \mathrm{GC}$ & 5 \\
\hline 39 & & 1 & & 15 th gr. & $\begin{array}{l}\text { 1 AIS Tchr/Testing } \\
\text { Coordtr } \\
1 \text { K-5 Math Coach/ } \\
\text { Cluster Teacher } \\
1 \text { Literacy Coach }\end{array}$ & & 5 \\
\hline 40 & 1 & 1 & $\begin{array}{l}1 \\
\text { (AIS point/ } \\
\text { staff dvlpr) }\end{array}$ & 1 Teacher & $\begin{array}{l}\text { 1 Speech/Lang } \\
\text { Pathologist }\end{array}$ & & 5 \\
\hline 41 & 1 & 1 & $\begin{array}{l}1 \\
\text { (Unit } \\
\text { Crdntr) }\end{array}$ & $\begin{array}{l}1 \mathrm{~K}-2 \text { SPED } \\
13-4 \text { SPED } \\
14-5 \text { SPED }\end{array}$ & & & 6 \\
\hline
\end{tabular}


Formative Study of the NYC Inquiry Team Process, 2007-08 


\section{Appendix E}

\section{Team Progress Toward June 2008 Learning Goals}

\begin{tabular}{|c|c|c|c|}
\hline \multicolumn{4}{|c|}{$\begin{array}{l}\text { Percent of Target Population Students in Study Schools Who Met or Almost Met } \\
\text { Their June } 2008 \text { Learning Goals }(n=38)\end{array}$} \\
\hline School & $\begin{array}{l}\text { Total number of } \\
\text { target population students }\end{array}$ & $\begin{array}{l}\text { Percent of students } \\
\text { who met goal }\end{array}$ & $\begin{array}{l}\text { Percent of students who } \\
\text { met or almost met goal }\end{array}$ \\
\hline 1 & 15 & $13 \%$ & $100 \%$ \\
\hline 2 & 38 & $55 \%$ & $100 \%$ \\
\hline 3 & 18 & $72 \%$ & $100 \%$ \\
\hline 4 & 16 & $75 \%$ & $100 \%$ \\
\hline 5 & 18 & $83 \%$ & $100 \%$ \\
\hline 6 & 12 & $92 \%$ & $100 \%$ \\
\hline 7 & 15 & $93 \%$ & $100 \%$ \\
\hline 8 & 16 & $94 \%$ & $100 \%$ \\
\hline 9 & 18 & $94 \%$ & $100 \%$ \\
\hline 10 & 16 & $100 \%$ & $100 \%$ \\
\hline 10 & 13 & $100 \%$ & $100 \%$ \\
\hline 11 & 30 & $87 \%$ & $97 \%$ \\
\hline 12 & 23 & $83 \%$ & $96 \%$ \\
\hline 13 & 19 & $63 \%$ & $95 \%$ \\
\hline 14 & 16 & $88 \%$ & $94 \%$ \\
\hline 15 & 31 & $74 \%$ & $90 \%$ \\
\hline 16 & 31 & $87 \%$ & $90 \%$ \\
\hline 17 & 20 & $85 \%$ & $90 \%$ \\
\hline 18 & 16 & $69 \%$ & $88 \%$ \\
\hline 19 & 15 & $60 \%$ & $87 \%$ \\
\hline 20 & 15 & $73 \%$ & $87 \%$ \\
\hline 21 & 15 & $73 \%$ & $87 \%$ \\
\hline 22 & 15 & $87 \%$ & $87 \%$ \\
\hline 23 & 20 & $80 \%$ & $85 \%$ \\
\hline 24 & 24 & $75 \%$ & $83 \%$ \\
\hline 25 & 16 & $69 \%$ & $81 \%$ \\
\hline 26 & 12 & $68 \%$ & $79 \%$ \\
\hline 27 & 19 & $68 \%$ & $79 \%$ \\
\hline 28 & 18 & $50 \%$ & $78 \%$ \\
\hline 29 & 16 & $56 \%$ & $75 \%$ \\
\hline 30 & 16 & $63 \%$ & $75 \%$ \\
\hline 31 & 15 & $33 \%$ & $73 \%$ \\
\hline 32 & 15 & $60 \%$ & $73 \%$ \\
\hline 33 & 25 & $28 \%$ & $68 \%$ \\
\hline 34 & 15 & $33 \%$ & $67 \%$ \\
\hline
\end{tabular}




\begin{tabular}{|c|c|c|c|}
\hline \multicolumn{4}{|c|}{ Percent of Target Population Students in Study Schools Who Met or Almost Met } \\
Their June 2008 Learning Goals (n=38)
\end{tabular}




\section{Appendix F \\ Department Plans to Strengthen the Inquiry Team Process in 2008-09}

\section{Department of Education Plans for 2008-09 for the Inquiry Team Process}

\section{Continue funding school-based Data Specialists and the Senior Achievement Facilitators (SAFs).}

The DoE plans to fund Data Specialists at the same level as in 2007-08 and has increased its investment in Senior Achievement Facilitators to bring the total number of SAFs from 62 to 64 . We recognize that both of these positions have been integral to the success of the inquiry work.

\section{Request that SSOs align their support and professional development to better support the work of school-based Inquiry Teams.}

We have restructured the planning and design of the Inquiry Team work to encourage significant customization at SSO level. The customization allows for the inquiry work to better align with the instructional agenda of the SSO. This alignment will increase the involvement of Network Leaders and teams in the instructional change strategies and will mitigate against the feeling that inquiry is separate from other school improvement activities happening in a school, Network or SSO.

\section{Develop a broad system of resources to support teams as they consider change strategies to use with their target population.}

During this summer, the DoE has worked with American Institutes for Research (AIR) to design a set of tools to help Inquiry Team members and other educators to help select an instructional strategy for their struggling students. AIR has been developing resource maps to help educators think through variables at the school, classroom, and student levels, specific to identified learning challenges in ELA and Math. This database of userfriendly abstracts, summarizing research will be able to assist with one of the key steps of the inquiry work - to design and implement an instructional change strategy with the target population. The AIR database will be accessible from ARIS.

Additionally, the Inquiry Team Interface captured the instructional change strategies employed by Inquiry Teams in 2007-08. The Interface is has a Google-like search feature that allows educators to quickly find the change strategies used by other schools working with similar student populations. The school can then call email, or visit the school with a promising practice. SAFs have used the Interface in many professional development sessions with Inquiry Team members.

In 2008-09, we plan to provide support for networks to form Inquiry Clusters, groups of six to eight schools within the same network that are grouped according to key structural variables that analytics have revealed to be strong predictors of student achievement- 
e.g., school size and higher concentration of lower performing students. Each cluster includes a "Beat the Odds" (BTO) school, which will serve as a peer mentor, leading the cluster in aligned inquiry. By grouping schools according to common challenges, Inquiry Clusters can facilitate collaboration, knowledge building, and diffusion of learning.

In 2007-08, SAFs coordinated focused inter-visitations between schools that received an "A" on their Progress Report and a "well-developed" on their Quality Review with schools that received a "D" of "F" on their Progress Report inside their Network. The visit focused on the strategies that the host school found most effective in leading to their success. These visits helped to share promising instructional strategies.

In 2007-08, many Networks hosted Inquiry Team "Share Fairs," where schools talked about their key learnings with other schools in the Network. We will continue to encourage this Network-level sharing.

Finally, an enhanced version of ARIS will launch in fall 2008 and will provide a data warehouse and analytic tools that will be available to Inquiry Team members.

\section{Reinforce the value of diverse teams that draw from multiple role groups in the school, namely classroom teachers, nonclassroom-based instructional coaches, and administrators.}

We agree that the core of inquiry work happens through classroom teachers. To this end, we would like to engage teachers as many teachers as possible in inquiry work. To achieve this, we will focus on infusing inquiry into existing professional collaboration in schools and will target outreach and support about the inquiry process to all teachers.

\section{Inquiry team chairs, Data Specialists and external support staff should help teams anticipate problem areas at different points in the process.}

Because SAFs have supported schools through a year of inquiry work, they will be able to prioritize and target their support around the common issues encountered in the work. Centrally, and at the SSO- and Network-level, we have more materials and resources created to assist schools in navigating the inquiry process. Inquiry Teams can support each other, too, now that some members at each school will be experienced.

\section{Develop an ongoing multipurpose approach to networking schools around the Inquiry Team process.}

The DoE is developing a robust Knowledge Management system to foster and disseminate local innovation by helping educators. Specifically, we hope the knowledge building and sharing initiative will help schools

- identify, share, and adopt effective educational practices;

- develop networks and communities of practice to collaborate and share expertise; and 
- develop a shared knowledge base of proven practices and resources to support ongoing innovation and improvement systemwide.

Currently, the process of innovation and adoption of new practices occurs largely within individual schools. We are designing a way to effectively share what adults in each school learn every day about moving students forward. Networks of schools emerge as an especially important resource for facilitating collaboration and knowledge sharing.

Networks also enable schools to gain the benefits both of empowerment and of scale. They are a logical locus for content-area expertise, technical support, and research and analytics capacity. By devolving these functions - alongside financial resources and decision-making authority — closer to the schools, the DOE can provide the focused supports educators need to accelerate student achievement.

We plan to launch two Network enhancement strategies in 2008-09.

1. Practice Area Networks (PANs): Schools join a network to collaborate around a particular strategy for accelerating student achievement. PANs will provide increased content expertise and research and analytics support, enabling member schools to engage in systematic analysis and diagnosis of students' learning needs, and support the experimental design and piloting of promising practices. Through their networks, PAN schools will coordinate their Inquiry Team process es to gain benefits of scale and facilitate knowledge building and sharing within and beyond the network. PANs will be organized around a practice area-a defining approach to accelerate student achievement.

2. Inquiry Clusters (referenced above).

Both strategies share a common goal: to facilitate advanced knowledge building and sharing within a network of schools.

Additionally, the Inquiry Team Interface provides a central repository where Inquiry Team members can record their experiences with their Inquiry Team work and search for information about the work of other teams. This resource helps SAFs and SSO staff members encourage knowledge sharing and networking around common skills, common goals, or other inquiry topics. The Inquiry Team Interface is located at http://childrenfirstintensive.com

As mentioned above, we will also continue to encourage Network "Share Fairs."

7. Provide more concrete guidance about good practice related to the different decision points of the Inquiry Team process and more advice about the pace of the Inquiry Team work that will enable completion of a full cycle in a single school year. 
We have created and distributed the Inquiry Team Handbook that lays out the conceptual underpinnings, goals, purpose and key steps in the inquiry work. The Inquiry Team Handbook also includes case studies at each step of the process, as well as a detailed guide to the assessments, tools, and resources available to help Teams make datainformed decisions. This document is available online at http://childrenfirstintensive.com/handbook

At the end of the 2007-08 school year, we videotaped several high-performing Inquiry Teams in action. The videos showed the Inquiry Team members at meeting, working with the target population students, and reflecting on their own work. The videos not only document the inquiry work, they also help struggling schools to understand the power of the approach. We are working to make these videos available online to all schools.

Additionally, the CFI Sharepoint site (http://cfi.sharepointsite.net) provides materials created by the CFI team, SAFs, and schools to scaffold and support the inquiry work.

\section{Focus additional resources on strengthening the professional cultures of schools with little or no history of collaborative exchanges among teachers or between administration and faculty.}

We are working with SAFs to stress the need to prioritize support to the schools with the least capacity to undertake the inquiry work. SAFs would particularly support these schools by focusing on

- supporting principals in empowering teachers to make the critical decisions for their students;

- involving principals and teachers in the SSO Design Team planning.

o These principals would provide targeted assistance to other principals needing a model for inquiry; and

- looking at existing structures in the school where inquiry could take place. 
Formative Study of the NYC Inquiry Team Process, 2007-08

\section{Nondiscrimination Statement}

The University of Pennsylvania values diversity and seeks talented students, faculty and staff from diverse backgrounds. The University of Pennsylvania does not discriminate on the basis of race, sex, sexual orientation, religion, color, national or ethnic origin, age, disability, or status as a Vietnam Era Veteran or disabled veteran in the administration of educational policies, programs or activities; admissions policies, scholarships or loan awards; athletic, or University administered programs or employment. Questions or complaints regarding this policy should be directed to Executive Director, Office of Affirmative Action, 1133 Blockley Hall, Philadelphia, PA 19104-6021 or (215) 898-6993 (Voice) or (215) 898-7803 (TDD). 